\title{
Theory and applications of atomic and ionic polarizabilities
}

\author{
J. Mitroy \\ School of Engineering, Charles Darwin University, Darwin NT 0909, Australia \\ M. S. Safronova \\ Department of Physics and Astronomy, University of Delaware, Newark, Delaware, 19716, USA \\ Charles W. Clark \\ Joint Quantum Institute, National Institute of Standards and Technology \\ and the University of Maryland, Gaithersburg, Maryland, 20899-8410, USA
}

(Dated: April 22, 2010)

\begin{abstract}
Atomic polarization phenomena impinge upon a number of areas and processes in physics. The dielectric constant and refractive index of any gas are examples of macroscopic properties that are largely determined by the dipole polarizability. When it comes to microscopic phenomena, the existence of alkaline-earth anions and the recently discovered ability of positrons to bind to many atoms are predominantly due to the polarization interaction. An imperfect knowledge of atomic polarizabilities is presently looming as the largest source of uncertainty in the new generation of optical frequency standards. Accurate polarizabilities for the group I and II atoms and ions of the periodic table have recently become available by a variety of techniques. These include refined many-body perturbation theory and coupled-cluster calculations sometimes combined with precise experimental data for selected transitions, microwave spectroscopy of Rydberg atoms and ions, refractive index measurements in microwave cavities, ab initio calculations of atomic structures using explicitly correlated wave functions, interferometry with atom beams, and velocity changes of laser cooled atoms induced by an electric field. This review examines existing theoretical methods of determining atomic and ionic polarizabilities, and discusses their relevance to various applications with particular emphasis on cold-atom physics and the metrology of atomic frequency standards.
\end{abstract}

PACS numbers: 31.15.ap, 32.10.Dk, 42.50.Hz, 51.70.+f

\section{INTRODUCTION}

By the time Maxwell presented his article on a Dynamical Theory of the Electromagnetic Field [1], it was understood that bulk matter had a composition of particles of opposite electrical charge, and that an applied electric field would rearrange the distribution of those charges in an ordinary object. This rearrangement could be described accurately even without a detailed microscopic understanding of matter. For example, if a perfectly conducting sphere of radius $r_{0}$ is placed in a uniform electric field $\mathbf{F}$, simple potential theory shows that the resulting electric field at a position $\mathbf{r}$ outside the sphere must be $\mathbf{F}-\nabla\left(\mathbf{F} \cdot \mathbf{r} r_{0}^{3} / r^{3}\right)$. This is equivalent to replacing the sphere with a point electric dipole,

$$
\mathbf{d}=\alpha \mathbf{F},
$$

where $\alpha=r_{0}^{3}$ is the dipole polarizability of the sphere $^{1}$. An arbitrary applied electric field can be decomposed into multipole fields of the form $\mathbf{F}_{q}^{k}(\mathbf{r})=$ $-F_{q}^{k} \nabla\left(r^{k} \mathbf{C}_{q}^{k}(\hat{\mathbf{r}})\right)$, where $\mathbf{C}_{q}^{k}(\hat{\mathbf{r}})$ is a spherical tensor [2]. Each of these will induce a multipole moment of $F_{q}^{k} r_{0}^{2 k+1}$

\footnotetext{
1 For notational convenience, we use the Gaussian system of electrical units, as discussed in subsection A below. In the Gaussian system, electric polarizability has the dimensions of volume.
}

in the conducting sphere, corresponding to a multipole polarizability of $\alpha^{k}=r_{0}^{2 k+1}$. Treatment of the electrical polarizabilities of macroscopic bodies is a standard topic of textbooks on electromagnetic theory, and the only material properties that it requires are dielectric constants and conductivities.

Quantum mechanics, on the other hand, offers a fundamental description of matter, incorporating the effects of electric and magnetic fields on its elementary constituents, and thus enables polarizabilities to be calculated from first principles. The standard framework for such calculations, perturbation theory, was first laid out by Schrödinger [3] in a paper that reported his calculations of the Stark effect in atomic hydrogen. A system of particles with positions $\mathbf{r}_{i}$ and electric charges $q_{i}$ exposed to a uniform electric field, $(\mathbf{F}=F \hat{\mathbf{F}})$, is described by the Hamiltonian

$$
H=H_{0}-F \hat{\mathbf{F}} \cdot \mathbf{d},
$$

where $H_{0}$ is the Hamiltonian in the absence of the field, and $\mathbf{d}$ is the dipole moment operator,

$$
\mathbf{d}=\sum_{i} q_{i} \mathbf{r}_{i}
$$

Treating the field strength, $F=|\mathbf{F}|$, as a perturbation parameter, means that the energy and wave function can 
be expanded as

$$
\begin{aligned}
|\Psi\rangle & =\left|\Phi_{0}\right\rangle+F\left|\Phi_{1}\right\rangle+F^{2}\left|\Phi_{2}\right\rangle+\ldots \\
E & =E_{0}+F E_{1}+F^{2} E_{2}+\ldots
\end{aligned}
$$

The first-order energy $E_{1}=0$ if $\left|\Phi_{0}\right\rangle$ is an eigenfunction of the parity operator. In this case, $\left|\Phi_{1}\right\rangle$ satisfies the equation

$$
\left(H_{0}-E_{0}\right)\left|\Phi_{1}\right\rangle=-\hat{\mathbf{F}} \cdot \mathbf{d}\left|\Phi_{0}\right\rangle .
$$

From the solution to Eq. (6), we can find the expectation value

$$
\begin{aligned}
\langle\Psi|\mathbf{d}| \Psi\rangle & =F\left(\left\langle\Phi_{0}|\mathbf{d}| \Phi_{1}\right\rangle+\left\langle\Phi_{1}|\mathbf{d}| \Phi_{0}\right\rangle\right) \\
& =\bar{\alpha} \mathbf{F}
\end{aligned}
$$

where $\bar{\alpha}$ is a matrix. The second-order energy is given by

$$
E_{2}=-\frac{1}{2} \mathbf{F} \cdot \bar{\alpha} \mathbf{F} .
$$

Although Eq. (6) can be solved directly, and in some cases in closed form, it is often more practical to express the solution in terms of the eigenfunctions and eigenvalues of $H_{0}$, so that Eq. (8) takes the form

$$
E_{2}=-\sum_{n} \frac{\left|\left\langle\Psi_{0}|\mathbf{d} \cdot \mathbf{F}| \Psi_{n}\right\rangle\right|^{2}}{E_{n}-E_{0}} .
$$

This sum over all stationary states shows that calculation of atomic polarizabilities is a demanding special case of the calculation of atomic structure. The sum extends in principle over the continuous spectrum, which sometimes makes substantial contributions to the polarizability.

Interest in the subject of polarizabilities of atomic states has recently been elevated by the appreciation that the accuracy of next-generation atomic time and frequency standards, based on optical transitions [4 9], is significantly limited by the displacement of atomic energy levels due to universal ambient thermal fluctuations of the electromagnetic field: blackbody radiation (BBR) shifts [10 13]. This phenomenon brings the most promising approach to a more accurate definition of the unit of time, the second, into contact with deep understanding of the thermodynamics of the electromagnetic radiation field.

Description of the interplay between these two fundamental phenomena is a major focus of this review, which in earlier times might have seemed a pedestrian discourse on atomic polarizabilities. The precise calculation of atomic polarizabilities also has implications for quantum information processing and optical cooling and trapping schemes. Modern requirements for precision and accuracy have elicited renewed attention to methods of accurate first-principles calculations of atomic structure, which recently have been increased in scope and precision by developments in methodology, algorithms, and raw computational power. It is expected that the future will lead to an increased reliance on theoretical treatments to describe the details of atomic polarization. Indeed, at the present time, many of the best estimates of atomic polarizabilities are derived from a composite analysis which integrates experimental measurements with first principles calculations of atomic properties.

There have been a number of reviews and tabulations of atomic and ionic polarizabilities [14 25]. Some of these reviews, e.g. [16, 17, 22, 23] have largely focussed upon experimental developments while others [19, 21, 25] have given theory more attention.

In the present review, the strengths and limitations of different theoretical techniques are discussed in detail given their expected importance in the future. Discussion of experimental work is mainly confined to presenting a compilation of existing results and very brief overviews of the various methods. The exception to this is the interpretation of resonance excitation Stark ionization spectroscopy [23] since issues pertaining to the convergence of the perturbation analysis of the polarization interaction are important here. The present review is confined to discussing the polarizabilities of low lying atomic and ionic states despite the existence of a body of research on Rydberg states [26]. High-order polarizabilities are not considered except in those circumstances where they are specifically relevant to ordinary polarization phenomenon. The influence of external electric fields on energy levels comprises part of this review as does the nature of the polarization interaction between charged particles with atoms and ions. The focus of this review is on developments related to contemporary topics such as development of optical frequency standards, quantum computing, and study of fundamental symmetries. Major emphasis of this review is to provide critically evaluated data on atomic polarizabilities. Table \ summarizes the data presented in this review to facilitate the search for particular information.

\section{A. Systems of units}

Dipole polarizabilities are given in a variety of units, depending on the context in which they are determined. The most widely used unit for theoretical atomic physics is atomic units (a.u.), in which, $e, m_{e}, 4 \pi \epsilon_{0}$ and the reduced Planck constant $\hbar$ have the numerical value 1 . The polarizability in a.u. has the dimension of volume, and its numerical values presented here are thus expressed in units of $a_{0}^{3}$, where $a_{0} \approx 0.052918 \mathrm{~nm}$ is the Bohr radius. The preferred unit systems for polarizabilities determined by experiment are $\AA^{3}, \mathrm{kHz} /(\mathrm{kV} / \mathrm{cm})^{2}$, $\mathrm{cm}^{3} / \mathrm{mol}$ or $\mathrm{C} \cdot \mathrm{m}^{2} / \mathrm{N}$ where $\mathrm{C} \cdot \mathrm{m}^{2} / \mathrm{N}$ is the SI unit. In this review, almost all polarizabilities are given in a.u. with uncertainties in the last digits (if appropriate) given 
TABLE I: List of data tables.

\begin{tabular}{|c|c|c|c|c|}
\hline Table & System & Atoms and Ions & States & Data \\
\hline TableIV & Noble gases & $\begin{array}{l}\mathrm{He}, \mathrm{Ne}, \mathrm{Ar}, \mathrm{Kr}, \mathrm{Xe}, \mathrm{Rn}, \mathrm{Li}^{+}, \mathrm{Na}^{+}, \mathrm{K}^{+}, \mathrm{Rb}^{+}, \mathrm{Cs}^{+}, \mathrm{Fr}^{+} \\
\mathrm{Be}^{2+}, \mathrm{Mg}^{2+}, \mathrm{Ca}^{2+}, \mathrm{Sr}^{2+}, \mathrm{Ba}^{2+}, \mathrm{Ra}^{2+}\end{array}$ & ground & $\alpha_{0}$ \\
\hline Table V & Alkali atoms & $\mathrm{Li}, \mathrm{Na}, \mathrm{K}, \mathrm{Rb}, \mathrm{Cs}, \mathrm{Fr}$ & ground $n s, n p$ & $\alpha_{0}, \alpha_{2}$ \\
\hline Table VI & Alkali ions & $\mathrm{Be}^{+}, \mathrm{Mg}^{+}, \mathrm{Ca}^{+}, \mathrm{Sr}^{+}, \mathrm{Ba}^{+}, \mathrm{Ra}^{+}$ & ground & $\alpha_{0}$ \\
\hline Table VIII & Monovalent & $\mathrm{Li}, \mathrm{Na}, \mathrm{K}, \mathrm{Ca}^{+}, \mathrm{Rb}, \mathrm{Sr}^{+}$ & excited & $\alpha_{0}, \alpha_{2}$ \\
\hline Table VII & Alkali atoms & Resonance transition: $\mathrm{Li}, \mathrm{Na}, \mathrm{K}, \mathrm{Rb}$, Cs & $n s, n p$ & $\Delta \alpha_{0}$ \\
\hline Table IX & Alkali atom & $\mathrm{Na}$ & ground & $\alpha_{0}$ \\
\hline Table $\mathrm{X}$ & Alkali atom & Cs & 26 states & $\alpha_{0}, \alpha_{2}$ \\
\hline Table XI & Group II type & $\mathrm{Be}, \mathrm{Mg}, \mathrm{Ca}, \mathrm{Sr}, \mathrm{Ba}, \mathrm{Ra}, \mathrm{Al}^{+}, \mathrm{Si}^{2+}, \mathrm{Zn}, \mathrm{Cd}, \mathrm{Hg}, \mathrm{Yb}$ & ground, $n s n p^{3} P_{0}$ & $\alpha_{0}$ \\
\hline Table XII & Miscellaneous & $\begin{array}{l}\mathrm{Al}, \mathrm{Ga}, \mathrm{In}, \mathrm{Tl}, \mathrm{Si}, \mathrm{Sn}, \mathrm{Pb}, \mathrm{Ir}, \mathrm{U}, \mathrm{Cu}, \mathrm{Ag}, \mathrm{Au} \\
\mathrm{Al}^{+}, \mathrm{Si}^{3+}, \mathrm{P}^{3+}, \mathrm{Kr}^{6+}, \mathrm{Cu}^{+}, \mathrm{Ag}^{+}, \mathrm{Hg}^{+}, \mathrm{Yb}^{+}, \mathrm{Zn}^{+}\end{array}$ & ground & $\alpha_{0}$ \\
\hline Table XIII & Miscellaneous & $\mathrm{Ca}, \mathrm{Sr}, \mathrm{Ba}, \mathrm{Zn}, \mathrm{Cd}, \mathrm{Hg}, \mathrm{Yb}, \mathrm{Al}, \mathrm{Tl}, \mathrm{Yb}^{+}$ & excited & $\alpha_{0}, \alpha_{2}$ \\
\hline Table XIV & Miscellaneous & $\mathrm{Li}, \mathrm{Na}, \mathrm{Cs}, \mathrm{Mg}, \mathrm{Ca}, \mathrm{Ba}, \mathrm{Hg}, \mathrm{Ga}, \mathrm{Tl}, \mathrm{Yb}^{+}$ & & $\Delta \alpha_{0}$ \\
\hline Table XVI & Miscellaneous & $\begin{array}{l}\mathrm{Mg}, \mathrm{Ca}, \mathrm{Sr}, \mathrm{Yb}, \mathrm{Zn}, \mathrm{Cd}, \mathrm{Hg} \\
\mathrm{Ca}^{+}, \mathrm{Sr}^{+}, \mathrm{Hg}^{+}, \mathrm{Yb}^{+}, \mathrm{Al}^{+}, \mathrm{In}^{+}\end{array}$ & $\begin{array}{l}\text { clock } \\
\text { transition }\end{array}$ & $\Delta \nu_{\mathrm{BBR}}$ \\
\hline Table XVIII & Monovalent & $\mathrm{Li}, \mathrm{Na}, \mathrm{K}, \mathrm{Rb}, \mathrm{Cs}, \mathrm{Ba}^{+}, \mathrm{Yb}^{+}, \mathrm{Hg}^{+}$ & ground hyperfine & $\mathrm{BBR}$ \\
\hline Table XIX & Alkali atoms & $\mathrm{Li}, \mathrm{Na}, \mathrm{K}, \mathrm{Rb}, \mathrm{Cs}, \mathrm{Fr}$ & ground & $C_{6}$ \\
\hline
\end{tabular}

in parentheses. Conversion factors between the different units are listed in Table II. The last line of the table gives conversion factors from SI units to the other units. For example, the atomic units for $\alpha$ can be converted to SI units by multiplying by 0.248832 .

Stark shift experiments which measure the change in photon frequency of an atomic transition as a function of electric field strength are usually reported as a Stark shift coefficient in units of $\mathrm{kHz} /(\mathrm{kV} / \mathrm{cm})^{2}$. The polarizability difference is twice the size of the Stark shift coefficient, as in equation (8).

\section{ATOMIC POLARIZABILITIES AND FIELD-ATOM INTERACTIONS}

\section{A. Static electric polarizabilities}

1. Definitions of scalar and tensor polarizabilities

The overall change in energy of the atom can be evaluated within the framework of second-order perturbation theory. Upon reduction, the perturbation theory expression given by Eq. (9) leads to a sum-over-states formula for the static scalar electric-dipole polarizability which is expressed most compactly in terms of oscillator strengths as

$$
\alpha_{0}=\sum_{n} \frac{f_{g n}}{\left(\Delta E_{n g}\right)^{2}} .
$$

In this expression, $f_{g n}$ is the absorption oscillator strength for a dipole transition from level $g$ to level $n$, defined in a $J$-representation as [27]

$$
f_{g n}=\frac{2\left|\left\langle\psi_{g}\left\|r \mathbf{C}^{1}(\hat{\mathbf{r}})\right\| \psi_{n}\right\rangle\right|^{2} \Delta E_{n g}}{3\left(2 J_{g}+1\right)},
$$

where $\Delta E_{n q}=E_{n}-E_{g}$ and $\mathbf{C}^{1}(\hat{\mathbf{r}})$ is the spherical tensor of rank 1 [2]. The definition of the oscillator strength in $L S$ coupling is transparently obtained from Eq. (11) by replacing the total angular momentum by the orbital angular momentum.

The polarizability for a state with non-zero angular momentum $J$ depends on the magnetic projection $M$ :

$$
\alpha=\alpha_{0}+\alpha_{2} \frac{3 M^{2}-J(J+1)}{J(2 J-1)} .
$$

The quantity $\alpha_{0}$ is called the scalar polarizability while $\alpha_{2}$ is the tensor polarizability in $\mathrm{J}$ representation.

The scalar part of the polarizability can be determined using Eq. (10). In terms of the reduced matrix elements of the electric-dipole operator, the scalar polarizability $\alpha_{0}$ of an atom in a state $\psi$ with total angular momentum 
TABLE II: Factors for converting polarizabilities between different unit systems. The table entries give the multiplying factor needed to convert the row entry to the corresponding column entry. The last column in the Table is the polarizability per mole and is often called the molar polarizability. The conversion factors from SI units to other units are given in the last line. Here, $h$ is the Planck's constant, $\epsilon_{0}$ is the electric constant, $a_{0}$ is the Bohr radius, and $N_{A}$ is the Avogadro constant.

\begin{tabular}{lccccc}
\hline \hline & a.u. & $\AA^{3}$ & $\mathrm{kHz} /(\mathrm{kV} / \mathrm{cm})^{2}$ & $\mathrm{C} \cdot \mathrm{m}^{2} / \mathrm{V}$ & $\mathrm{cm}^{3} / \mathrm{mol}^{-41}$ \\
\hline a.u. & 1 & 0.1481847 & 0.2488319 & $1.648773 \times 10^{-41}$ & 0.3738032 \\
$\AA^{3}$ & 6.748335 & 1 & 1.679201 & $1.112650 \times 10^{-40}$ & 2.522549 \\
$\mathrm{kHz} /(\mathrm{kV} / \mathrm{cm})^{2}$ & 4.018778 & 0.5955214 & 1 & $1.509190 \times 10^{40}$ & 1.502232 \\
$\mathrm{Cm}^{2} / \mathrm{V}$ & $6.065100 \times 10^{40}$ & $8.987552 \times 10^{39}$ & $6.626069 \times 10^{-39}$ & 1 & $2.267154 \times 10^{40}$ \\
$\mathrm{~cm}^{3} / \mathrm{mol}$ & 2.675205 & 0.3964244 & 0.6656762 & $4.410816 \times 10^{-41}$ & 1 \\
Conversion from SI & $1 /\left(4 \pi \epsilon_{0} a_{0}^{3}\right)$ & $10^{30} /\left(4 \pi \epsilon_{0}\right)$ & $10^{-7} h$ & 1 & $10^{6} N_{A} /\left(3 \epsilon_{0}\right)$ \\
\hline \hline
\end{tabular}

$J$ and energy $E$ is also written as

$$
\alpha_{0}=\frac{2}{3(2 J+1)} \sum_{n} \frac{\left|\left\langle\psi\left\|r \mathbf{C}^{1}(\hat{\mathbf{r}})\right\| \psi_{n}\right\rangle\right|^{2}}{E_{n}-E} .
$$

The tensor polarizability $\alpha_{2}$ is defined as

$$
\begin{aligned}
& \alpha_{2}=4\left(\frac{5 J(2 J-1)}{6(J+1)(2 J+1)(2 J+3)}\right)^{1 / 2} \\
& \times \sum_{n}(-1)^{J+J_{n}}\left\{\begin{array}{ccc}
J & 1 & J_{n} \\
1 & J & 2
\end{array}\right\} \frac{\mid\left\langle\psi\left\|r \mathbf{C}^{1}(\hat{\mathbf{r}})\right\| \psi_{n} \mid\right\rangle^{2}}{E_{n}-E} .
\end{aligned}
$$

It is useful in some cases to calculate polarizabilities in strict $L S$ coupling. In such cases [28], the tensor polarizability $\alpha_{2, L}$ for a state with orbital angular momentum $\mathrm{L}$ is given by

$$
\begin{aligned}
\alpha_{2, L} & =\sum_{n}\left[\left(\begin{array}{ccc}
L & 1 & L_{n} \\
-L & 0 & L
\end{array}\right)^{2}-\frac{1}{3(2 L+1)}\right] \\
& \times \frac{2\left|\left\langle\psi\left\|r \mathbf{C}^{1}(\hat{\mathbf{r}})\right\| \psi_{n}\right\rangle\right|^{2}}{E-E_{n}} .
\end{aligned}
$$

The tensor polarizabilities $\alpha_{2}$ and $\alpha_{2, L}$ in the $J$ and $L$ representations, respectively, are related by

$$
\begin{aligned}
\alpha_{2} & =\alpha_{2, L}(-1)^{S+L+J+2}(2 J+1)\left\{\begin{array}{ccc}
S & L & J \\
2 & J & L
\end{array}\right\} \\
& \times\left(\begin{array}{ccc}
J & 2 & J \\
-J & 0 & J
\end{array}\right)\left(\begin{array}{ccc}
L & 2 & L \\
-L & 0 & L
\end{array}\right)^{-1} .
\end{aligned}
$$

For $L=1$ and $J=3 / 2$, Eq. (16) gives $\alpha_{2}=\alpha_{2, L}$. For $L=1, S=1$ and $J=1$, Eq. (16) gives $\alpha_{2}=-\alpha_{2, L} / 2$. For $L=2, \alpha_{2}=(7 / 10) \alpha_{2, L}$ for $J=3 / 2$ and $\alpha_{2}=\alpha_{2, L}$ for $J=5 / 2$. We use the shorter $\left\langle\psi\|D\| \psi_{n}\right\rangle$ designation for the reduced electric-dipole matrix elements instead of $\left\langle\psi\left\|r \mathbf{C}^{1}(\hat{\mathbf{r}})\right\| \psi_{n}\right\rangle$ below.

Equation (14) indicates that spherically symmetric levels (such as the $6 s_{1 / 2}$ and $6 p_{1 / 2}$ levels of cesium) only have a scalar polarizability. However, the hyperfine states of these levels can have polarizabilities that depend upon the hyperfine quantum numbers $F$ and $M_{F}$. The relationship between $F$ and $J$ polarizabilities is discussed in Ref. [29]. This issue is discussed in more detail in the section on BBR shifts.

There are two distinctly different broad approaches to the calculation of atomic polarizabilities. The "sum-overstates" approach uses a straightforward interpretation of Eq. (9) with the contribution from each state $\Psi_{n}$ being determined individually, either from a first principles calculation or from interpretation of experimental data. A second class of approaches solves inhomogeneous equation (6) directly. We refer to this class of approaches as direct methods, but note that there are many different implementations of this strategy.

\section{The sum-over-states method}

The sum-over-states method utilizes expression such as Eqs. (10, 13 - 15) to determine the polarizability. This approach is widely used for systems with one or two valence electrons since the polarizability is often dominated by transitions to a few low lying excited states. The sumover-states approach can be used with oscillator strengths (or electric-dipole matrix elements) derived from experiment or atomic structure calculations. It is also possible to insert high-precision experimental values of these quantities into an otherwise theoretical determination of the total polarizability. For such monovalent or divalent systems, it is computationally feasible to explicitly construct a set of intermediate states that is effectively complete. Such an approach is computationally more difficult to apply for atoms near the right hand side of the periodic table since the larger dimensions involved would preclude an explicit computation of the entire set of intermediate state wave functions.

For monovalent atoms, it is convenient to separate the total polarizability of an atom into the core polarizability $\alpha_{\text {core }}$ and the valence part defined by Eq. (13). The core contribution actually has two components, the polarizability of the ionic core and a small change due to the presence of the valence electron [30]. For the alkali 
atoms, the valence part of the ground state polarizability is completely dominated by the contribution from the lowest excited state. For example, the $5 s-5 p_{1 / 2}$ and $5 s-5 p_{3 / 2}$ transitions contribute more than $99 \%$ of the $\mathrm{Rb}$ valence polarizability [31]. The $\mathrm{Rb}^{+}$core polarizability contributes about $3 \%$. Therefore, precision experimental measurements of the transition rates for the dominant transitions can also be used to deduce accurate values of the ground state polarizability. However, this is not the case for some excited states where several transitions may have large contributions and continuum contributions may be not negligible.

This issue is illustrated using the polarizability of the $5 p_{1 / 2}$ state of the $\mathrm{Rb}$ atom [30], which is given by

$$
\begin{aligned}
\alpha_{0}\left(5 p_{1 / 2}\right) & =\frac{1}{3} \sum_{n} \frac{\left|\left\langle n s\|D\| 5 p_{1 / 2}\right\rangle\right|^{2}}{E_{n s}-E_{5 p_{1 / 2}}} \\
& +\frac{1}{3} \sum_{n} \frac{\left|\left\langle n d_{3 / 2}\|D\| 5 p_{1 / 2}\right\rangle\right|^{2}}{E_{n d_{3 / 2}}-E_{5 p_{1 / 2}}}+\alpha_{\text {core }}
\end{aligned}
$$

We present a solution to the Eq. (17) that combines first principles calculations with experimental data. The strategy to produce a high-quality recommended value with this approach is to calculate as many terms as realistic or feasible using the high-precision atomic structure methods. Where experimental high-precision data are available (for example, for the $5 s-5 p$ transitions) they are used in place of theory, assuming that the expected theory uncertainty is higher than that of the experimental values. The remainder that contains contributions from highly-excited states is generally evaluated using (Dirac-Hartree-Fock) DHF or random-phase approximation (RPA) methods. In our example, the contribution from the very high discrete $(n>10)$ and continuum states is about $1.5 \%$ and cannot be omitted in a precision calculation. Table III lists the dipole matrix elements and energy differences required for evaluation of Eq. (17) as well as the individual contributions to the polarizability. Experimental values from [32] are used for the $5 s-5 p_{j}$ matrix elements, otherwise the matrix elements are obtained from the all-order calculations of Ref. [30] described in Section IVF. Absolute values of the matrix elements are given. Experimental energies from [33, 34] are used. Several transitions give significant contributions. This theoretical number agrees with experimental measurement within the uncertainty. The comparison with experiment is discussed in Section V.

\section{Direct methods}

From a conceptual viewpoint, the finite-field method represents one of the simplest ways to compute the polarizability. In this approach, one solves the Schrödinger equation using standard techniques for the perturbed Hamiltonian given by Eq. (2) for a variety of values of $F$. The polarizability is then extracted from the dipole mo-
TABLE III: The contributions (in a.u.) to the scalar polarizability of the $\mathrm{Rb}$ atom in the $5 p_{1 / 2}$ state [30]. The uncertainties in each term are enclosed in parenthesis. The corresponding energy differences $\Delta E=E_{n}-E_{5 p_{1 / 2}}$ [34] are given in $\mathrm{cm}^{-1}$, which can be converted to atomic units by division by 219474.6. Experimental values from [32] are used for absolute values of the $5 s-5 p_{j}$ matrix elements, otherwise the matrix elements are obtained from all-order calculations of Ref. [30].

\begin{tabular}{lcrr}
\hline \hline Contribution & $\left|\left\langle n\|D\| 5 p_{1 / 2}\right\rangle\right|$ & \multicolumn{1}{c}{$\Delta E$} & \multicolumn{1}{c}{$\alpha_{0}\left(5 p_{1 / 2}\right)$} \\
\hline $5 p_{1 / 2}-5 s$ & 4.231 & -12579 & $-104.11(15)$ \\
$5 p_{1 / 2}-6 s$ & 4.146 & 7554 & $166.5(2.2)$ \\
$5 p_{1 / 2}-7 s$ & 0.953 & 13733 & $4.835(16)$ \\
$5 p_{1 / 2}-8 s$ & 0.502 & 16468 & $1.120(7)$ \\
$5 p_{1 / 2}-9 s$ & 0.331 & 17920 & $0.448(3)$ \\
$5 p_{1 / 2}-10 s$ & 0.243 & 18783 & $0.230(2)$ \\
$5 p_{1 / 2}-11 s$ & 0.189 & 19338 & $0.135(1)$ \\
$5 p_{1 / 2}-(12-\infty) s$ & & & $1.9(0.2)$ \\
$5 p_{1 / 2}-4 d_{3 / 2}$ & & & \\
$5 p_{1 / 2}-5 d_{3 / 2}$ & 8.017 & 6777 & $694(30)$ \\
$5 p_{1 / 2}-6 d_{3 / 2}$ & 1.352 & 13122 & $10.2(9)$ \\
$5 p_{1 / 2}-7 d_{3 / 2}$ & 1.067 & 16108 & $5.2(1.1)$ \\
$5 p_{1 / 2}-8 d_{3 / 2}$ & 0.787 & 17701 & $2.6(4)$ \\
$5 p_{1 / 2}-9 d_{3 / 2}$ & 0.605 & 18643 & $1.4(2)$ \\
$5 p_{1 / 2}-(10-\infty) d_{3 / 2}$ & 0.483 & 19243 & $0.89(10)$ \\
$\alpha_{\text {core }}$ & & & $10.5(10.5)$ \\
Total & & & $9.08(45)$ \\
\hline \hline
\end{tabular}

ment or the energy eigenvalues of the perturbed Hamiltonian. This usually entails doing a number of calculations at different discrete field strengths. This approach is generally used to obtain polarizabilities in coupledcluster calculations (see, for example, Refs. [35, 36]). We note that linearized coupled-cluster calculations are implemented very differently, and sum-over-states is used for the polarizability calculations [37]. These differences between coupled-cluster calculations are discussed in Section IV

Another direct approach to calculating polarizability is the perturbation-variation method [38]. The perturbation-variation approach has been outlined in the introduction as Eqs. (5) to (7). The unperturbed state, $\left|\Phi_{0}\right\rangle$ and perturbed state, $\left|\Phi_{1}\right\rangle$ would be written as a linear combinations of basis states. Equations (6) and (7) then reduce to sets of matrix equations. A general technique for solving the inhomogeneous equation (6) has been described by Dalgarno and Lewis in Ref. [39].

Exact solutions to Eqs. (5) - (7) are possible for atomic hydrogen and hydrogenic ions. The non-relativistic solutions were first obtained independently in 1926 by Epstein [40], Waller [41], and Wentzel [42]; the relativistic case remains a subject of current research interest [43 46]. The nonrelativistic equations are separable in 
parabolic coordinates, and the polarizability of a hydrogenic ion of nuclear charge $\mathrm{Z}$ in the state $\mid n_{1} n_{2} m>$ is (in a.u.)

$$
\alpha=\frac{n^{4}}{8 Z^{4}}\left[17 n^{2}-3\left(n_{1}-n_{2}\right)^{2}-9 m^{2}+19\right] a_{0}^{3},
$$

where $n_{1}, n_{2}$ are parabolic quantum numbers [47], $m$ is the projection of the orbital angular momentum onto the direction of the electric field, and $n=n_{1}+n_{2}+|m|+1$ is the principal quantum number. A convenient special case is $n=|m|+1$, which corresponds to the familiar circular states of hydrogen in spherical coordinates, with orbital angular momentum $l=|m|=n-1$; for these states, $\alpha=(|m|+2)(|m|+9 / 4) a_{0}^{3}$.

For the $\mathrm{H} 1 s$ ground state exposed to an electric field $\mathbf{F}=F \hat{\mathbf{z}}$, the solution to Eq. (6) is (in a.u.)

$$
\begin{aligned}
& \Phi_{0}=e^{-r} / \sqrt{\pi}, \\
& \Phi_{1}=-z(1+r / 2) \Phi_{0},
\end{aligned}
$$

from which $\alpha=(9 / 2) a_{0}^{3}$. Note that although $\Phi_{1}$ of Eq. (20) is a $p$ state, it is much more compact than any of the discrete $n p$ eigenstates of $\mathrm{H}$. Thus building up $\Phi_{1}$ by the sum-over-states approach requires a significant contribution from the continuous spectrum of $\mathrm{H}$. This is depicted in Fig. 11, which employs the histogram construction of Fano and Cooper [48] to show the connection between discrete and continuum contributions to the sum over states. About $20 \%$ of the polarizability of $\mathrm{H} 1 s$ comes from the continuum.

FIG. 1: Solid line: histogram representation of the sum-overstates contributions to the polarizability of $\mathrm{H} 1 s$. Following Ref. [48], the contributions of discrete states (e.g. $2 p$ ) are spread over the inverse density of states, to show continuity with the continuum contributions near energy $E=0$. The polarizability, $\alpha$, is equal to the area under this curve. Dashed line: the same construction, for an electron bound to a onedimensional delta-function potential with energy $E=-1 / 2$ a.u. From [49].

Clearly, the direct solution of the Schrödinger equation for an atom in the presence of an electric field and subsequent determination of the polarizability is formally equivalent to the sum-over-states approach described in the previous subsection. However, it is useful to comment on how this equivalence is actually seen in calculations for many-electron atoms. For example, randomphase-approximation (RPA) results for polarizabilities of closed-shell atoms [50] that were obtained by direct solution of inhomogeneous equation are the same (up to numerical uncertainty of the calculations) as sum-overstate RPA results obtained using formula

$$
\alpha_{\text {core }}=\frac{2}{3} \sum_{m a} \frac{\left|\left\langle\psi_{a}\left\|D^{\mathrm{DHF}}\right\| \psi_{m}\right\rangle\left\langle\psi_{a}\left\|D^{\mathrm{RPA}}\right\| \psi_{m}\right\rangle\right|}{E_{m}-E_{a}},
$$

where $\left\langle\psi_{a}\left\|D^{\mathrm{DHF}}\right\| \psi_{m}\right\rangle$ is reduced matrix element of dipole operator obtained in the DHF approximation and the $\left\langle\psi_{a}\left\|D^{\mathrm{RPA}}\right\| \psi_{m}\right\rangle$ matrix elements include RPA terms using many-body perturbation theory as discussed, for example, in [51]. The index $a$ refers to all core orbitals, while the $m$ includes all other orbitals. The sum-overstates can be calculated with a finite basis set [52], and such an approach intrinsically includes the continuum states when complete sum over the entire basis set is carried out. When the contributions from highly-excited states are significant, it becomes difficult to account for these terms accurately within the framework of the sumover-states approach. Direct method automatically accounts for these states and this problem does not arise. However, it becomes difficult and cumbersome to include corrections to the dipole operator beyond RPA. The method implemented in [50] is different from the finite field approach and does not involve performing a number of calculations at different discrete field strengths.

In most high-precision calculations, the determination of polarizabilities follows the calculation of wave functions or quantities that represent the wave functions (such as excitation coefficients). The type of approach used for this initial calculation generally determines whether polarizabilities are determined by Eqs. (6) or by sum-over-states method. For example, relativistic linearized coupled-cluster approach [37] is formulated in a way that does not explicitly generate numerical wave functions on a radial grid, and all quantities are expressed in terms of excitation coefficients. Therefore, the polarizabilities are calculated by the sum-over-states method using resulting high-quality dipole matrix elements and energies. In the case of methods that combine relativistic configuration interaction and perturbation theory [CI+MBPT], it is natural to determine polarizabilities by directly solving the inhomogeneous equation. In this case, it is solved in the valence space with the effective operators that are determined using MBPT [53]. The ionic core polarizability is calculated separately in this approach. The effective dipole operator generally includes RPA corrections, with other corrections calculated independently.

The direct and sum-over-states approaches can also be merged in a hybrid approach. One strategy is to perform 
a direct calculation using the best available techniques, and then replace the transition matrix elements for the most important low-lying states with those from a higher level theory. This hybrid method is discussed further in the sections on the CI+MBPT and CI+all-order methods.

\section{B. The frequency-dependent polarizability}

So far, we have described the polarizability for static fields. The numerical value of the polarizability changes when the atom is immersed in an alternating (AC) electromagnetic field. To second order, one writes $\Delta E=$ $-\frac{1}{2} \alpha(\omega) F^{2}+\ldots$ The valence part of the scalar frequency dependent polarizability, usually called the dynamic polarizability, is calculated using the sum-overstates approach with a straightforwardly modified version of Eq. (13):

$$
\alpha_{0}(\omega)=\frac{2}{3(2 J+1)} \sum_{n} \frac{\Delta E\left|\left\langle\psi\|D\| \psi_{n}\right\rangle\right|^{2}}{(\Delta E)^{2}-\omega^{2}}
$$

Eq. (22) assumes that $\omega$ is at least a few linewidths away from resonant frequencies defined by $\Delta E=E_{n}-E$. As noted previously, atomic units are used throughout this paper, and $\hbar=1$. The core part of the polarizability may also be corrected for frequency dependence in random phase approximation by similarly modifying the formula (21). Static values may be used for the core contribution in many applications since the frequencies of interest, (i.e. corresponding to commonly used lasers) are very far from the excitation energies of the core states. The calculations of the ground and excited state frequencydependent polarizabilities of the alkali-metal atoms are described in detail in Refs. [54] and [29], respectively. It is essentially the same as the calculation of the static polarizability described in Section IA2, only for $\omega \neq 0$.

The expression for the tensor polarizability given by Eq. (14) is modified in the same way, i.e. by replacing

$$
\frac{1}{\Delta E} \rightarrow \frac{\Delta E}{\Delta E^{2}-\omega^{2}}
$$

There has been more interest recently in the determination of frequency-dependent polarizabilities due to the need to know various "magic wavelengths" 55] for the development of optical frequency standards and other applications. At such wavelengths, the frequencydependent polarizabilities of two states are the same, and the AC Stark shift of the transition frequency between these two states is zero. An example of the calculation of frequency-dependent polarizabilities and magic wavelengths is given in Section VIIB. Experimentally determined magic wavelengths may also be used to gauge the accuracy of the theory.

\section{MEASUREMENTS OF POLARIZABILITIES AND RELATED QUANTITIES}

Experimental measurements of atomic and ionic polarizabilities are somewhat rarer than theoretical determinations. There are two types of measurements, those which directly determine the polarizability, and those which determine differences in polarizabilities of two states from Stark shift of atomic transitions.

For the most part, we make brief comments on the major experimental techniques and refer the reader to primarily experimental reviews [17, 19, 22, 23] for further details.

\section{A. $f$-sum rules}

This approach makes use of Eqs. (10,15). Many of the most interesting atoms used in cold atom physics typically have only one or two valence electrons. The ground state polarizability of these atoms is dominated by a single low-lying transition. As mentioned in Section II A 2 , $97 \%$ of the total value of $\mathrm{Rb}$ ground state polarizability comes from $5 s \rightarrow 5 p$ transition. In the case of $\mathrm{Na}$, about $99.4 \%$ of the valence polarizability and $98.8 \%$ of the total polarizability of sodium arises from the $3 s \rightarrow 3 p$ resonant transition.

Composite estimates of the polarizability using both experimental and theoretical inputs are possible. One type of estimate would use experimental oscillator strengths to determine the valence polarizability. This could be combined with a core contribution obtained by other methods to estimate the total polarizability. Another approach replaces the most important matrix elements in a first-principles calculation by high precision experimental values [56, 57]. Various types of experiments may be used to determine particular matrix elements, including photo-association experiments [58], lifetime, oscillator strengths, or Stark shift measurements [30] with photoassociation experiments generally giving the most reliable matrix elements. This hybrid method may provide values accurate to better than $0.5 \%$ in certain cases [56].

\section{B. Dielectric constant}

The dielectric constant $K$ of an atomic or molecular gas is related to the dipole polarizability, $\alpha$, by the identity

$$
\alpha=\frac{K-1}{4 \pi N}
$$

where $N$ is the atomic number density. The technique has only been applied to the rare gas atoms, and the nitrogen and oxygen atoms by the use of a shock tube. Results for the rare gases typically achieve precisions of $0.01-0.1 \%$. Examples are reported in Table IV. 


\section{Refractive index}

The frequency-dependent refractive index of a gas $n(\omega)$, is related to the polarizability by the expression

$$
\alpha(\omega)=\frac{n(\omega)-1}{2 \pi N}
$$

where $N$ is the atomic number density. The static dipole polarizability, $\alpha(0)$, can be extracted from the frequencydependent polarizability $\alpha(\omega)$ by the following technique.

The energy denominator in Eq. (22) can be expanded when the frequency is smaller than the frequency of the first excitation giving

$$
\alpha(\omega)=\alpha(0)+\omega^{2} S(-4)+\omega^{4} S(-6) \ldots
$$

The $S(-q)$ factors are the Cauchy moments of the oscillator strength distribution and are defined by

$$
S(-q)=\sum_{n} \frac{f_{g n}}{\Delta E_{n g}^{q}} .
$$

Specific Cauchy moments arise in a number of atomic physics applications, as reviewed by Fano and Cooper [48]. For example, the Thomas-Reiche-Kuhn sum rule states that $S(0)$ is equal to the number of electrons in the atom. The $S(-3)$ moment is related to the non-adiabatic dipole polarizability [59, 60].

The general functional dependence of the polarizability at low frequencies is given by Eq. (26) 61, 62]. The achievable precision for the rare gases is $0.1 \%$ or better [62, 63]. Experiments on the vapours of $\mathrm{Zn}, \mathrm{Cd}$ and $\mathrm{Hg}$ gave polarizabilities with uncertainties of $1-10 \%$ [64, 65].

\section{Deflection of an atom beam by electric fields}

The beam deflection experiment is conceptually simple. A collimated atomic beam is directed through an interaction region containing an inhomogeneous electric field. While the atom is in the interaction region, the electric field $\mathbf{F}$ induces a dipole moment on the atom. Since the field is not uniform, a force proportional to the gradient of the electric field and the induced dipole moment results in the deflection of the atomic beam. The polarizability is deduced from the deflection of the beam. The overall uncertainty in the derived polarizabilities is between $5-10 \%$ [66]. Therefore, this method is mainly useful at the present time for polarizability measurements in atoms inaccessible by any other means.

\section{E. The $E-H$ balance method}

In this approach, the $E$ - $H$ balance configuration applies an inhomogeneous electric field and an inhomogeneous magnetic field in the interaction region [67]. The magnetic field acts on the magnetic moment of the atom giving a magnetic deflection force in addition to the electric deflection. The experiment is tuned so that the electric and magnetic forces are in balance. The polarizability can be determined since the magnetic moments of many atoms are known. Uncertainties range from $2 \%$ to $10 \%$ 67 69].

\section{F. Atom interferometry}

The interferometry approach splits the beam of atoms so that one path sends a beam through a parallel plate capacitor while the other goes through a field free region. An interference pattern is then measured when the beams are subsequently merged and detected. The polarizability is deduced from the phase shift of the beam passing through the field free region. So far, this approach has been used to measure the polarizabilities of helium (see 70] for a discussion of this measurement), lithium 71], sodium 72, 73], potassium 73], and rubidium [73] achieving uncertainties of $0.35-0.8 \%$.

It has been suggested that multi-species interferometers could possibly determine the polarizability ratio $R=\alpha_{X} / \alpha_{\mathrm{Y}}$ to $10^{-4}$ relative accuracy [70]. Consequently, a measurement of $R$ in conjunction with a known standard, say lithium, could lead to a new level of precision in polarizability measurements. Already the Na:K and $\mathrm{Na}: \mathrm{Rb}$ polarizability ratios have been measured with a precision of $0.3 \%$ 73.

\section{G. Cold atom velocity change}

The experiment of Amini and Gould 74 measured the kinetic energy gained as cold cesium atoms were launched from a magneto-optical trap into a region with a finite electric field. The kinetic energy gained only depends on the final value of the electric field. The experimental arrangement actually measures the time of return for cesium atoms to fall back after they are launched into a region between a set of parallel electric-field plates. The only such experiment reported so far gave a very precise estimate of the Cs ground state polarizability, namely $\alpha_{0}=401.0(6)$ a.u.. This approach can in principle be applied to measure the polarizability of many other atoms with a precision approaching $0.1 \%$ [22].

\section{H. Other approaches}

The deflection of an atomic beam by pulsed lasers has been used to obtain the dynamic polarizabilities of rubidium and uranium [75, 76]. The dynamic polarizabilities of some metal atoms sourced from an exploding wire have been measured interferometrically [77, 78]. These approaches measure polarizabilities to an accuracy of 5$20 \%$. 


\section{Spectral analysis for ion polarizabilities}

The polarizability of an ion can in principle be extracted from the energies of non-penetrating Rydberg series of the parent system[41, 79, 80]. The polarizability of the ionic core leads to a shift in the $(n, L)$ energy levels away from their hydrogenic values.

Consider a charged particle interacting with an atom or ion at large distances. To zeroth order, the interaction potential between a highly excited electron and the residual ion is just

$$
V(r)=\frac{Z-N}{r},
$$

where $Z$ is the nuclear charge and $N$ is the number of electrons. However, the outer electron perturbs the atomic charge distribution. This polarization of the electron charge cloud leads to an attractive polarization potential between the external electron and the atom. The Coulomb interaction in a multipole expansion with $|\mathbf{r}|>|\mathbf{x}|$, is written as

$$
\frac{1}{|\mathbf{r}-\mathbf{x}|}=\sum_{k} \mathbf{C}^{k}(\mathbf{x}) \cdot \mathbf{C}^{k}(\mathbf{r}) \frac{x^{k}}{r^{k+1}}
$$

Applying second-order perturbation theory leads to the adiabatic polarization potential between the charged particle and the atom, e.g.

$$
V_{\mathrm{pol}}(r)=-\sum_{k=1}^{\infty} \frac{\alpha^{E k}}{2 r^{2 k+2}} .
$$

The quantities $\alpha^{E k}$ are the multipole polarizabilities defined as

$$
\alpha^{E k}=\sum_{n} \frac{f_{g n}^{(k)}}{\left(\Delta E_{g n}\right)^{2}} .
$$

In this notation, the electric-dipole polarizability is written as $\alpha^{E 1}$, and $f_{g n}^{(k)}$ is the absorption oscillator strength for a multipole transition from $g \longrightarrow n$. Equation (30), with its leading term involving the dipole polarizability is not absolutely convergent in $k$ [81]. At any finite $r$, continued summation of the series given by Eq. (30), with respect to $k$, will eventually result in a divergence in the value of the polarization potential.

Equation (30) is modified by non-adiabatic corrections [59, 60]. The non-adiabatic dipole term is written as

$$
V_{\text {non-ad }}=\frac{6 \beta_{0}}{2 r^{6}},
$$

where the non-adiabatic dipole polarizability, $\beta_{0}$ is defined

$$
\beta_{0}=\sum \frac{f_{g n}^{(1)}}{2\left(\Delta E_{g n}\right)^{3}}
$$

The non-adiabatic interaction is repulsive for atoms in their ground states. The polarization interaction includes further adiabatic, non-adiabatic and higher order terms that contribute at the $r^{-7}$ and $r^{-8}$, but there has been no systematic study of what could be referred to as the non-adiabatic expansion of the polarization potential.

When the Rydberg electron is in a state that has negligible overlap with the core (this is best achieved with the electron in high angular momentum orbitals), then the polarization interaction usually provides the dominant contribution to this energy shift. Suppose the dominant perturbation to the long-range atomic interaction is

$$
V_{\mathrm{pol}}(r)=-\frac{C_{4}}{r^{4}}-\frac{C_{6}}{r^{6}},
$$

where $C_{4}=\alpha_{0} / 2$ and $C_{6}=\left(\alpha_{0}-6 \beta\right) / 2$. Equation (34) omits the $C_{7} / r^{7}$ and $C_{8} / r^{8}$ terms that are included in a more complete description $[82$ 84]. The energy shift due to an interaction of this type can be written

$$
\frac{\Delta E}{\Delta\left\langle r^{-4}\right\rangle}=C_{4}+C_{6} \frac{\Delta\left\langle r^{-6}\right\rangle}{\Delta\left\langle r^{-4}\right\rangle}
$$

where $\Delta E$ is usually the energy difference between two Rydberg states. The expectation values $\Delta\left\langle r^{-6}\right\rangle$ and $\Delta\left\langle r^{-4}\right\rangle$ are simply the differences in the radial expectations of the two states. These are easily evaluated using the identities of Bockasten [85]. Plotting $\frac{\Delta E}{\Delta\left\langle r^{-4}\right\rangle}$ versus $\frac{\Delta\left\langle r^{-6}\right\rangle}{\Delta\left\langle r^{-4}\right\rangle}$ yields $C_{4}$ as the intercept and $C_{6}$ as the gradient. Such a graph is sometimes called a polarization plot.

Traditional spectroscopies such as discharges or laser excitation find it difficult to excite atoms into Rydberg states with $L>6$. Exciting atoms into states with $L>6$ is best done with resonant excitation Stark ionization spectroscopy (RESIS) 23]. RESIS spectroscopy first excites an atomic or ionic beam into a highly-excited state, and then uses a laser to excite the system into a very highly-excited state which is Stark ionized.

While this approach to extracting polarizabilities from Rydberg series energy shifts is appealing, there are a number of perturbations that act to complicate the analysis. These include relativistic effects $\Delta E_{\text {rel }}$, Stark shifts from ambient electric fields $\Delta E_{\mathrm{ss}}$, second-order effects due to relaxation of the Rydberg electron in the field of the polarization potential $\Delta E_{\text {sec }}$ 86 88], and finally the corrections due to the $C_{7} / r^{7}$ and $C_{8} / r^{8}$ terms, $\Delta E_{7}, \Delta E_{8}$, and $\Delta E_{8 L}$. Therefore, the energy shift between two neighbouring Rydberg states is

$$
\begin{aligned}
\Delta E & =\Delta E_{4}+\Delta E_{6}+\Delta E_{7}+\Delta E_{8}+\Delta E_{8 L} \\
& +\Delta E_{\mathrm{rel}}+\Delta E_{\mathrm{sec}}+\Delta E_{\mathrm{ss}}
\end{aligned}
$$

One way to solve the problem is to simply subtract these 
terms from the observed energy shift, e.g.

$$
\begin{aligned}
\frac{\Delta E_{c}}{\Delta\left\langle r^{-4}\right\rangle} & =\frac{\Delta E_{\mathrm{obs}}}{\Delta\left\langle r^{-4}\right\rangle} \\
& -\left(\frac{\Delta E_{\mathrm{rel}}+\Delta E_{\mathrm{sec}}+\Delta E_{s s}}{\Delta\left\langle r^{-4}\right\rangle}\right) \\
& -\left(\frac{\Delta E_{7}+\Delta E_{8}+\Delta E_{8 L}}{\Delta\left\langle r^{-4}\right\rangle}\right) .
\end{aligned}
$$

and then deduce $C_{4}$ and $C_{6}$ from the polarization plot of the corrected energy levels [84].

\section{J. Stark shift measurements of polarizability differences}

The Stark shift experiment predates the formulation of quantum mechanics in its modern form [89]. An atom is immersed in an electric field, and the shift in wavelength of one of its spectral lines is measured as a function of the field strength. Stark shift experiments effectively measure the difference between the polarizability of the two atomic states involved in the transition. Stark shifts can be measured for both static and dynamic electric fields. While there have been many Stark shift measurements, relatively few have achieved an overall precision of $1 \%$ or better.

While the polarizabilities can generally be extracted from the Stark shift measurement, it is useful to compare the experimental values directly with theoretical predictions where high precision is achieved for both theory and experiment. In this review, comparisons of the theoretical static polarizability differences for the resonance transitions involving the alkali atoms with the corresponding Stark shifts are provided in Section V. Some of the alkali atom experiments report precisions between 0.01 and 0.1 a.u. 90 93]. The many Stark shift experiments involving Rydberg atoms 94 are not detailed here.

Selected Stark shifts for some non-alkali atoms that are of interest for applications described in this review are discussed in Section $\mathrm{V}$ as well. The list is restricted to low-lying excited states for which high precision Stark shifts are available. When compared with the alkali atoms, there are not that many measurements and those that have been performed have larger uncertainties.

The tensor polarizability of an open shell atom can be extracted from the difference in polarizabilities between the different magnetic sub-levels. Consequently, tensor polarizabilities do not rely on absolute polarizability measurements and can be extracted from Stark shift measurements by tuning the polarization of a probe laser. Tensor polarizabilities for a number of states of selected systems are discussed in Section $\nabla$

One unusual experiment was a measurement of the $\mathrm{AC}$ energy shift ratio for the $6 s$ and $5 d_{3 / 2}$ states of $\mathrm{Ba}^{+}$to an accuracy of $0.11 \%$ [95]. This experiment does not give polarizabilities, and is mainly valuable as an additional constraint upon calculation [96].

\section{K. AC Stark shift measurements}

There are few experimental measurements of AC Stark shifts at optical frequencies. Two recent examples would be the determination of the Stark shift for the $\mathrm{Al}^{+}$clock transition [97] and the Li $2 s-3 s$ Stark shifts [98] at the frequencies of the pump and probe laser of a two-photon resonance transition between the two states. One difficulty in the interpretation of AC Stark shift experiments is the lack of precise knowledge about the overlap of the laser beam with atoms in the interaction region. This is also a complication in the analysis of experiment on deflection of atomic beams by lasers [75, 76].

\section{PRACTICAL CALCULATION OF ATOMIC POLARIZABILITIES}

There have been numerous theoretical studies of atomic and ionic polarizabilities in the last several decades. Most methods used to determine atomic wave functions and energy levels can be adapted to generate polarizabilities. These have been divided into a number of different classes that are listed below. We give a brief description of each approach. It should be noted that the list is not exhaustive, and the emphasis here has been on those methods that have achieved the highest accuracy or those methods that have been applied to a number of different atoms and ions.

\section{A. Configuration interaction}

The configuration interaction (CI) method [99] and its variants are widely used for atomic structure calculations owing to general applicability of the CI method. The CI wave function is written as a linear combination of configuration state functions

$$
\Psi_{\mathrm{CI}}=\sum_{i} c_{i} \Phi_{i},
$$

i.e. a linear combination of Slater determinants from a model subspace 100. Each configuration is constructed with consideration given to anti-symmetrization, angular momentum and parity requirements. There is a great deal of variety in how the CI approach is implemented. For example, sometimes the exact functional form of the orbitals in the excitation space is generated iteratively during successive diagonalization of the excitation basis. Such a scheme is called the multi-configuration HartreeFock (MCHF) or multi-configuration self consistent field (MCSCF) approach [101]. The relativistic version of MCHF is referred to as multi-configuration Dirac-Fock (MCDF) method [102].

The CI approach has a great deal of generality since there are no restrictions imposed upon the virtual orbital space and classes of excitations beyond those limited 
by the computer resources. The method is particularly useful for open shell systems which contain a number of strongly interacting configurations. On the other hand, there can be a good deal of variation in quality between different CI calculations for the same system, because of the flexibility of introducing additional configuration state functions.

The most straightforward way to evaluate polarizability within the framework of the CI method it to use a direct approach by solving the inhomogeneous equation (6). RPA corrections to the dipole operator can be incorporated using the effective operator technique described in Section IVG It is also possible to use CIgenerated matrix elements and energies to evaluate sums over states. The main drawback of the CI method is its loss of accuracy for heavier systems. It becomes difficult to include a sufficient number of configurations for heavier systems to produce accurate results even with modern computer facilities. One solution of this problem is to use a semi-empirical core potential (CICP method) described in the next subsection. Another, ab initio solution, involves construction of the effective Hamiltonian using either many-body perturbation theory (CI+MBPT) or allorder linearized coupled-cluster method (CI+all-order) and carrying out CI calculations in the valence sector. These approaches are described in the last two sections of this chapter.

\section{B. CI calculations with a semi-empirical core potential (CICP)}

The $a b$ initio treatment of core-valence correlations greatly increases the complexity of any structure calculation. Consequently, to include this physics in the calculation, using a semi-empirical approach is an attractive alternative for an atom with a few valence electrons 103 105.

In this method, the active Hamiltonian for a system with two valence electrons is written as

$$
\begin{aligned}
H & =\sum_{i=1}^{2}\left(-\frac{1}{2} \nabla_{i}^{2}+V_{\mathrm{dir}}\left(\mathbf{r}_{\mathbf{i}}\right)+V_{\mathrm{exc}}\left(\mathbf{r}_{\mathbf{i}}\right)+V_{\mathrm{p} 1}\left(\mathbf{r}_{\mathbf{i}}\right)\right) \\
& +\frac{1}{r_{12}}+V_{\mathrm{p} 2}\left(\mathbf{r}_{1}, \mathbf{r}_{2}\right)
\end{aligned}
$$

The $V_{\text {dir }}$ and $V_{\text {exc }}$ represent the direct and exchange interactions with the core electrons. In some approaches, these terms are represented by model potentials, 106108]. More refined approaches evaluate $V_{\text {dir }}$ and $V_{\text {exc }}$ using core wave functions calculated with the Hartree-Fock (or Dirac-Fock) method [104, 105, 109]. The one-body polarization interaction $V_{\text {pol }}(r)$ is semi-empirical in nature and can be written in its most general form as an $\ell$-dependent potential, e.g.

$$
V_{\mathrm{p} 1}(\mathbf{r})=-\sum_{\ell m} \frac{\alpha g_{\ell}^{2}(r)}{2 r^{4}}|\ell m\rangle\langle\ell m|,
$$

where $\alpha$ is the static dipole polarizability of the core and $g_{\ell}^{2}(r)$ is a cutoff function that eliminates the $1 / r^{4}$ singularity at the origin. The cutoff functions usually include an adjustable parameter that is tuned to reproduce the binding energies of the valence states. The two-electron or di-electronic polarization potential is written

$$
V_{\mathrm{p} 2}\left(\mathbf{r}_{i}, \mathbf{r}_{j}\right)=-\frac{\alpha}{r_{i}^{3} r_{j}^{3}}\left(\mathbf{r}_{i} \cdot \mathbf{r}_{j}\right) g\left(r_{i}\right) g\left(r_{j}\right)
$$

There is variation between expressions for the core polarization potential, but what is described above is fairly representative. One choice for the cutoff function is $g_{\ell}^{2}(r)=1-\exp \left(-r^{6} / \rho_{\ell}^{6}\right)[105]$, but other choices exist.

A complete treatment of the core-polarization corrections also implies that corrections have to be made to the multipole operators [104, 105, 110, 111]. The modified transition operator is obtained from the mapping

$$
r^{k} \mathbf{C}^{k}(\mathbf{r}) \rightarrow g_{\ell}(r) r^{k} \mathbf{C}^{k}(\mathbf{r})
$$

Usage of the modified operator is essential to the correct prediction of the oscillator strengths. For example, it reduces the $\mathrm{K}(4 s \rightarrow 4 p)$ oscillator strength by $8 \%$ 104].

One advantage of this configuration interaction plus core-polarization (CICP) approach is in reducing the size of the calculation. The elimination of the core from active consideration permits very accurate solutions of the Schrödinger equation for the valence electrons. Introduction of the core-polarization potentials, $V_{p 1}$ and $V_{p 2}$, introduces an additional source of uncertainty into the calculation. However, this additional small source of uncertainty is justified by the almost complete elimination of computational uncertainty in the solution of the resulting simplified Schrödinger equation.

The CICP approach only gives the polarizability of the valence electrons. Core polarizabilities are typically quite small for the group I and II atoms, e.g. the cesium atom has a large core polarizability of about $15.6 a_{0}^{3}$ [112], but this represents only $4 \%$ of the total ground state polarizability of $401 a_{0}^{3}$ [74]. Hence, usage of moderate accuracy core polarizabilities sourced from theory or experiment will lead to only small inaccuracies in the total polarizability.

Most implementation of the CICP approach to the calculation of polarizabilities have been within a nonrelativistic framework. A relativistic variant (RCICP) has recently been applied to zinc, cadmium, and mercury [113. It should be noted that even non-relativistic calculations incorporate relativistic effects to some extent. Tuning the core polarization correction to reproduce the experimental binding energy partially incorporates relativistic effects on the wave function.

\section{Density functional theory}

Approaches based on Density Functional Theory (DFT) are not expected to give polarizabilities as accu- 
rate as those coming from the refined $a b$ initio calculations described in the following sections. Polarizabilities from DFT calculations are most likely to be useful for systems for which large scale $a b$ initio calculations are difficult, e.g. the transition metals. DFT calculations are often much less computationally expensive than $a b$ initio calculations. There have been two relatively extensive DFT compilations [114, 115] that have reported dipole polarizabilities for many atoms in the periodic table.

\section{Correlated basis functions}

The accuracy of atomic structure calculations can be dramatically improved by the use of basis functions which explicitly include the electron-electron coordinate. The most accurate calculations reported for atoms and ions with two or three electrons have typically been performed with exponential basis functions including the inter-electronic coordinates as a linear factor. A typical Hylleraas basis function for lithium would be

$$
\chi=r_{1}^{j_{1}} r_{2}^{j_{2}} r_{3}^{j_{3}} r_{12}^{j_{12}} r_{13}^{j_{13}} r_{23}^{j_{23}} \exp \left(-\alpha r_{1}-\beta r_{2}-\gamma r_{3}\right) .
$$

Difficulties with performing the multi-center integrals have effectively precluded the use of such basis functions for systems with more than three electrons. Within the framework of the non-relativistic Schrödinger equation, calculations with Hylleraas basis sets achieve accuracies of 13 significant digits [116] for polarizability of two-electron systems and 6 significant digits for the polarizability of three-electron systems [117, 118]. Inclusion of relativistic and quantum electrodynamic (QED) corrections to the polarizability of helium has been carried out in Refs. [116, 119], and the resulting final value is accurate to 7 significant digits.

Another correlated basis set that has recently found increasingly widespread use utilizes the explicitly correlated gaussian (ECG). A typical spherically symmetric explicitly correlated gaussian for a three-electron system is written as 120 .

$$
\chi=\exp \left(-\sum_{i=1}^{3} \alpha_{i} r_{i}^{2}-\sum_{i<j} \beta_{i j} r_{i j}^{2}\right) .
$$

The multi-center integrals that occur in the evaluation of the Hamiltonian can be generally reduced to analytic expressions that are relatively easy to compute. Calculations using correlated gaussians do not achieve the same precision as Hylleraas forms, but are still capable of achieving much higher precision than orbital based calculations provided the parameters $\alpha_{i}$ and $\beta_{i j}$ are well optimized [120, 121].

\section{E. Many-body perturbation theory}

The application of many-body perturbation theory (MBPT) is discussed in this section in the context of the Dirac equation. While MBPT has been applied with the non-relativistic Schrödinger equation, many recent applications most relevant to this review have been using a relativistic Hamiltonian.

The point of departure for the discussions of relativistic many-body perturbation theory (RMBPT) calculations is the no-pair Hamiltonian obtained from QED by Brown and Ravenhall [122], where the contributions from negative-energy (positron) states are projected out. The no-pair Hamiltonian can be written in second-quantized form as $H=H_{0}+V$, where

$$
\begin{aligned}
H_{0} & =\sum_{i} \epsilon_{i}\left[a_{i}^{\dagger} a_{i}\right], \\
V & =\frac{1}{2} \sum_{i j k l}\left(g_{i j k l}+b_{i j k l}\right)\left[a_{i}^{\dagger} a_{j}^{\dagger} a_{l} a_{k}\right] \\
& +\sum_{i j}\left(V_{\mathrm{DHF}}+B_{\mathrm{DHF}}-U\right)_{i j}\left[a_{i}^{\dagger} a_{j}\right],
\end{aligned}
$$

and a c-number term that just provides an additive constant to the energy of the atom has been omitted.

In Eqs. (45- 46), $a_{i}^{\dagger}$ and $a_{i}$ are creation and annihilation operators for an electron state $i$, and the summation indices range over electron bound and scattering states only. Products of operators enclosed in brackets, such as $\left[a_{i}^{\dagger} a_{j}^{\dagger} a_{l} a_{k}\right]$, designate normal products with respect to a closed core. The core DHF potential is designated by $V_{\mathrm{DHF}}$ and its Breit counterpart is designated by $B_{\mathrm{DHF}}$. The quantity $\epsilon_{i}$ in Eq. (45) is the eigenvalue of the Dirac equation. The quantities $g_{i j k l}$ and $b_{i j k l}$ in Eq. (46) are two-electron Coulomb and Breit matrix elements, respectively

$$
\begin{aligned}
g_{i j k l} & =\left\langle i j\left|\frac{1}{r_{12}}\right| k l\right\rangle, \\
b_{i j k l} & =-\left\langle i j\left|\frac{\boldsymbol{\alpha}_{1} \cdot \boldsymbol{\alpha}_{2}+\left(\boldsymbol{\alpha}_{1} \cdot \hat{\boldsymbol{r}}_{12}\right)\left(\boldsymbol{\alpha}_{2} \cdot \hat{\boldsymbol{r}}_{12}\right)}{2 r_{12}}\right| k l\right\rangle,
\end{aligned}
$$

where $\boldsymbol{\alpha}$ are Dirac matrices.

For neutral atoms, the Breit interaction is often a small perturbation that can be ignored compared to the Coulomb interaction. In such cases, it is particularly convenient to choose the starting potential $U(r)$ to be the core DHF potential $U=V_{\mathrm{DHF}}$,

$$
\left(V_{\mathrm{DHF}}\right)_{i j}=\sum_{a}\left[g_{i a j a}-g_{i a a j}\right],
$$

since with this choice, the second term in Eq. (46) vanishes. The index $a$ refers to all core orbitals. The Breit $\left(B_{\mathrm{DHF}}\right)_{i j}$ term is defined as

$$
\left(B_{\mathrm{DHF}}\right)_{i j}=\sum_{a}\left[b_{i a j a}-b_{i a a j}\right] .
$$


For monovalent atoms, the lowest-order wave function is written as

$$
\left|\Psi_{v}^{(0)}\right\rangle=a_{v}^{\dagger}\left|0_{c}\right\rangle
$$

where $\left|0_{c}\right\rangle=a_{a}^{\dagger} a_{b}^{\dagger} \cdots a_{n}^{\dagger}|0\rangle$ is the closed core wave function, $|0\rangle$ being the vacuum wave function, and $a_{v}^{\dagger}$ being a valence-state creation operator. The indices $a$ and $b$ refer to core orbitals.

The perturbation expansion for the wave function leads immediately to a perturbation expansion for matrix elements. Thus, for the one-particle operator written in the second-quantized form as

$$
Z=\sum_{i j} z_{i j} a_{i}^{\dagger} a_{j}
$$

perturbation theory leads to an order-by-order expansion for the matrix element of $Z$ between states $v$ and $w$ of an atom with one valence electron:

$$
\left\langle\Psi_{w}|Z| \Psi_{v}\right\rangle=Z_{w v}^{(1)}+Z_{w v}^{(2)}+\cdots
$$

The first-order matrix element is given by the DHF value in the present case

$$
Z_{w v}^{(1)}=z_{w v}
$$

The second-order expression for the matrix element of a one-body operator $Z$ in a Hartree-Fock potential is given by

$$
Z_{w v}^{(2)}=\sum_{a m} \frac{z_{a m} \widetilde{g}_{w m v a}}{\epsilon_{a v}-\epsilon_{m w}}+\sum_{a m} \frac{z_{m a} \widetilde{g}_{w a v m}}{\epsilon_{w a}-\epsilon_{m v}}
$$

where $\epsilon_{w a}=\epsilon_{w}+\epsilon_{a}$. The summation index $a$ ranges over states in the closed core, and the summation index $m$ ranges over the excited states. The complete third-order MBPT expression for the matrix elements of monovalent systems was given in Ref. [51]. The monumental task of deriving and evaluating the complete expression for the fourth-order matrix elements has been carried out for $\mathrm{Na}$ in Ref. [123.

The polarizabilities are obtained using a sum-overstate approach by combining the resulting matrix elements and either experimental or theoretical energies. The calculations are carried out with a finite basis set, resulting in a finite sum in the sum-over-state expression that it is equivalent to the inclusion of all bound states and the continuum. Third-order MBPT calculation of polarizabilities is described in detail, for example, in Ref. [124 for $\mathrm{Yb}^{+}$.

The relativistic third-order many-body perturbation theory generally gives good results for electric-dipole (E1) matrix elements of lighter systems in the cases when the correlation corrections are not unusually large. For example, the third-order value of the $\mathrm{Na} 3 s-3 p_{1 / 2}$ matrix element agrees with high-precision experiment to $0.6 \%$ [37]. However, the third-order values for the $6 s-6 p_{1 / 2}$ matrix element in Cs and $7 s-7 p_{1 / 2}$ matrix element in
Fr differ from the experimental data by $1.3 \%$ and $2 \%$, respectively [37]. For some small matrix elements, for example $6 s-7 p$ in $\mathrm{Cs}$, third-order perturbation theory gives much poorer values. As a result, various methods that are equivalent to summing dominant classes of perturbation theory terms to all orders have to be used to obtain precision values, in particular when sub-percent accuracy is required.

The relativistic all-order correlation potential method that enables efficient treatment of dominant core-valence correlations was developed in Ref. [125]. It was used to study fundamental symmetries in heavy atoms and to calculate atomic properties of alkali-metal atoms and isoelectronic ions (see, for example, Refs. [126, 127] and references therein). In the correlation potential method for monovalent systems, the calculations generally start from the relativistic Hartree-Fock method in the $V^{N-1}$ approximation. The correlations are incorporated by means of a correlation potential $\Sigma$ defined in such a way that its expectation value over a valence electron wave function is equal to the RMBPT expression for the correlation correction to the energy of the electron. Two classes of higher-order corrections are generally included in the correlation potential: the screening of the Coulomb interaction between a valence electron and a core electron by outer electrons, and hole-particle interactions. Ladder diagrams were included to all orders in Ref. [128]. The correlation potential is used to build a new set of single-electron states for subsequent evaluation of various matrix elements using the random-phase approximation. Structural radiation and the normalization corrections to matrix elements are also incorporated. This approach was used to evaluate black-body radiation shifts in microwave frequency standards in Refs. [129, 130] (see Section VII C 5).

Another class of the all-order approaches based on the coupled-cluster method is discussed in the next subsection.

\section{F. Coupled-cluster methods}

In the coupled-cluster method, the exact many-body wave function is represented in the form 131]

$$
|\Psi\rangle=\exp (S)\left|\Psi^{(0)}\right\rangle,
$$

where $\left|\Psi^{(0)}\right\rangle$ is the lowest-order atomic wave function. The operator $S$ for an N-electron atom consists of "cluster" contributions from one-electron, two-electron, $\cdots$, N-electron excitations of the lowest-order wave function $\left|\Psi^{(0)}\right\rangle: S=S_{1}+S_{2}+\cdots+S_{N}$. In the single-double approximation of the coupled-cluster (CCSD) method, only single and double excitation terms with $S_{1}$ and $S_{2}$ are retained. Coupled-cluster calculations which use a relativistic Hamiltonian are identified by a prefix of $\mathrm{R}$, e.g. RCCSD.

The exponential in Eq. (56), when expanded in terms 
of the $n$-body excitations $S_{n}$, becomes

$$
|\Psi\rangle=\left\{1+S_{1}+S_{2}+S_{3}+\frac{1}{2} S_{1}^{2}+S_{1} S_{2}+\cdots\right\}\left|\Psi^{(0)}\right\rangle .
$$

Actual implementations of the coupled-cluster approach and subsequent determination of polarizability vary significantly with the main source of variation being the inclusion of triple excitations or non-linear terms and use of different basis sets. These differences account for some discrepancies between different coupled-cluster calculations for the same system. It is common for triple excitations to be included perturbatively. In this review, all coupled-cluster calculations that include triples in some way are labelled as CCSDT (or RCCSDT, RLCCSDT) calculations with no further distinctions being made.

We can generally separate coupled-cluster calculations of polarizabilities into two groups, but note that details of calculations vary between different works. Implementations of the CCSDT method in the form typically used for the quantum chemistry calculations use gaussian type orbital basis sets. Care should be taken to explore the dependence of the final results on the choice and size of the basis set. The dependence of the dipole polarizability values on the quality of the basis set used has been discussed, for example, in Ref. 35]. In those calculations, the polarizabilities are generally calculated using the finite-field approach 35, 36, 132]. Consequently, such $\mathrm{CC}$ calculations are not restricted to monovalent systems, and RCC calculations of polarizabilities of divalent systems have been reported in Refs. [35, 133, 134.

The second type of relativistic coupled-cluster calculations is carried out using the linearized variant of the coupled-cluster method (referred to as the relativistic allorder method in most references), which was first developed for atomic physics calculations and applied to He in Ref. [135]. The extension of this method to monovalent systems was introduced in Ref. [136]. We refer to this approach as the RLCCSD or RLCCSDT method [37]. We note that RLCCSDT method includes only valence triples using perturbative approach. As noted above, all $\mathrm{CC}$ calculations that include triples in some way are labelled as CCSDT. The RLCCSDT method uses finite basis set of B-splines rather than gaussian orbitals. The Bspline basis sets are effectively complete for each partial wave, i.e. using a larger basis set will produce negligible changes in the results. The partial waves with $l=0-6$ are generally used. Third-order perturbation theory is used to account for higher partial waves where necessary. Very large basis sets are used, typically a total of 500 700 orbitals are included for monovalent systems. Therefore, this method avoids the basis set issues generally associated with other coupled-cluster calculations. The actual algorithm implementation is distinct from standard quantum chemistry codes as well.

In the linearized coupled-cluster approach, all nonlinear terms are omitted and the wave function takes the form

$$
|\Psi\rangle=\left\{1+S_{1}+S_{2}+S_{3}+\cdots+S_{N}\right\}\left|\Psi^{(0)}\right\rangle .
$$

The inclusion of the nonlinear terms within the framework of this method is described in Ref. [137]. Restricting the sum in Eq. (58) to single, double, and valence triple excitations yields the expansion for the wave function of a monovalent atom in state $v$ :

$$
\begin{aligned}
\left|\Psi_{v}\right\rangle & =\left[1+\sum_{m a} \rho_{m a} a_{m}^{\dagger} a_{a}+\frac{1}{2} \sum_{m n a b} \rho_{m n a b} a_{m}^{\dagger} a_{n}^{\dagger} a_{b} a_{a}+\right. \\
& +\sum_{m \neq v} \rho_{m v} a_{m}^{\dagger} a_{v}+\sum_{m n a} \rho_{m n v a} a_{m}^{\dagger} a_{n}^{\dagger} a_{a} a_{v} \\
& \left.+\frac{1}{6} \sum_{m n r a b} \rho_{m n r v a b} a_{m}^{\dagger} a_{n}^{\dagger} a_{r}^{\dagger} a_{b} a_{a} a_{v}\right]\left|\Psi_{v}^{(0)}\right\rangle
\end{aligned}
$$

where the indices $m, n$, and $r$ range over all possible virtual states while indices $a$ and $b$ range over all occupied core states. The quantities $\rho_{m a}, \rho_{m v}$ are single-excitation coefficients for core and valence electrons and $\rho_{m n a b}$ and $\rho_{\text {mnva }}$ are double-excitation coefficients for core and valence electrons, respectively, $\rho_{\text {mnrvab }}$ are the triple valence excitation coefficients. For the monovalent systems, $U$ is generally taken to be the frozen-core $V^{\mathrm{N}-1}$ potential, $U=V_{\mathrm{DF}}$.

We refer to results obtained with this approach as RLCCSDT, indicating inclusion of single, double, and partial triple excitations. The triple excitations are generally included perturbatively. Strong cancellations between groups of smaller terms, for example non-linear terms and certain triple excitation terms have been found in Ref. [138]. As a result, additional inclusion of certain classes of terms may not necessarily lead to more accurate values.

The matrix elements for any one-body operator $Z$ given in second-quantized form by Eq. (52) are obtained within the framework of the linearized coupled-cluster method as

$$
Z_{w v}=\frac{\left\langle\Psi_{w}|Z| \Psi_{v}\right\rangle}{\sqrt{\left\langle\Psi_{v} \mid \Psi_{v}\right\rangle\left\langle\Psi_{w} \mid \Psi_{w}\right\rangle}}
$$

where $\left|\Psi_{v}\right\rangle$ and $\left|\Psi_{w}\right\rangle$ are given by the expansion (59). In the SD approximation, the resulting expression for the numerator of Eq. (60) consists of the sum of the DHF matrix element $z_{w v}$ and 20 other terms that are linear or quadratic functions of the excitation coefficients [136]. The main advantage of this method is its general applicability to calculation of many atomic properties of ground and excited states: energies, electric and magnetic multipole matrix elements and other transition properties such as oscillator strengths and lifetimes, $A$ and $B$ hyperfine constants, dipole and quadrupole polarizabilities, parity-nonconserving matrix elements, electron electricdipole-moment (EDM) enhancement factors, $C_{3}$ and $C_{6}$ coefficients, etc. 
The all-order method yields results for the properties of alkali atoms 31] in excellent agreement with experiment. The application of this method to the calculation of alkali polarizabilities (using a sum-over-state approach) is described in detail in Refs. 29 31, 56].

In its present form described above, the RLCCSDT method is only applicable to the calculation of polarizabilities of monovalent systems. The work on combining the RLCCSDT approach with the CI method to create a method that is more general is currently in progress [139] and is described in Section IVH.

\section{G. Combined CI and many-body perturbation theory}

Precise calculations for atoms with several valence electrons require an accurate treatment of valence-valence correlations. While finite-order MBPT is a powerful technique for atomic systems with weakly interacting configurations, its accuracy can be limited when the wave function has a number of strongly interacting configurations. One example occurs for the alkaline-earth atoms where there is strong mixing between the $n s^{2}$ and $n p^{2}$ configurations of ${ }^{1} \mathrm{~S}$ symmetry. For such systems, an approach combining both aspects has been developed by Dzuba et al. 100] and later applied to the calculation of atomic properties of many other systems [53, 57, 140143]. This composite approach to the calculation of atomic structure is often abbreviated as CI+MBPT (we use RCI+MBPT designations in this review to indicate that the method is relativistic).

For systems with more than one valence electron, the precision of the CI method is drastically limited by the sheer number of the configurations that should be included. As a result, the core-core and core-valence correlations might only receive a limited treatment, which can lead to a significant loss of accuracy. The RCI+MBPT approach provides a complete treatment of core correlations to a limited order of perturbation theory. The $\mathrm{RCI}+\mathrm{MBPT}$ approach uses perturbation theory to construct an effective core Hamiltonian, and then a CI calculation is performed to generate the valence wave functions.

The no-pair Hamiltonian given by Eqs. (45) and (46) separates into a sum of the one-body and two-body interactions,

$$
H=H_{1}+H_{2},
$$

where $\mathrm{H}_{2}$ contains the Coulomb (or Coulomb + Breit) matrix elements $v_{i j k l}$. In the RCI+MBPT approach, the one-body term $H_{1}$ is modified to include a correlation potential $\Sigma_{1}$ that accounts for part of the core-valence correlations, $H_{1} \rightarrow H_{1}+\Sigma_{1}$. Either the second-order expression for $\Sigma_{1}^{(2)}$ or all-order chains of such terms can be used (see, for example, Ref. [100]). The two-body Coulomb interaction term in $\mathrm{H}_{2}$ is modified by including the two-body part of core-valence interaction that represents screening of the Coulomb interaction by valence electrons; $H_{2} \rightarrow H_{2}+\Sigma_{2}$. The quantity $\Sigma_{2}$ is calculated in second-order MBPT [100]. The CI method is then used with the modified $H_{\text {eff }}$ to obtain improved energies and wave functions.

The polarizabilities are determined using the direct approach (in the valence sector) by solving the inhomogeneous equation in the valence space, approximated from Eq. (6). For state $v$ with total angular momentum $J$ and projection $M$, the corresponding equation is written as 53 .

$$
\left(E_{v}-H_{\mathrm{eff}}\right)\left|\Psi\left(v, M^{\prime}\right)\right\rangle=D_{\mathrm{eff}}\left|\Psi_{0}(v, J, M)\right\rangle .
$$

The wave function $\Psi\left(v, M^{\prime}\right)$ is composed of parts that have angular momenta of $J^{\prime}=J, J \pm 1$. This then permits the scalar and tensor polarizability of the state $|v, J, M\rangle$ to be determined [53].

The construction of $H_{\text {eff }}$ was described in the preceding paragraphs. The effective dipole operator $D_{\text {eff }}$ includes random phase approximation (RPA) corrections and several smaller MBPT corrections described in [144]. NonRPA corrections may be neglected in some cases [53]. There are several variants of the RCI+MBPT method that differ by the corrections included in the effective operators $H_{\text {eff }}$ and $D_{\text {eff }}$, the functions used for the basis sets, and versions of the CI code. In some implementations of the RCI+MBPT, the strength of the effective Hamiltonian is rescaled to improve agreement with binding energies. However, this procedure may not necessarily improve the values of polarizabilities.

The contributions from the dominant transitions may be separated and replaced by more accurate experimental matrix elements when appropriate. Such a procedure is discussed in detail in Ref. 141]. This hybrid RCI+MBPT approach [13, 57, 145] has been used to obtain present recommended values for the polarizabilities of the $n s^{2}$ and $n$ snp ${ }^{3} P_{0}$ states of $\mathrm{Mg}, \mathrm{Ca}, \mathrm{Sr}, \mathrm{Hg}$, and $\mathrm{Yb}$ needed to evaluate the blackbody radiation shifts of the relevant optical frequency standards.

\section{H. Combined CI and all-order method}

The RCI+MBPT approach described in the previous section includes only a limited number of the core-valence excitation terms (mostly in second order) and deteriorates in accuracy for heavier, more complicated systems. The linearized coupled-cluster approach described in Section IVF is designed to treat core-core and core-valence correlations with high accuracy. As noted above, it is restricted in its present form to the calculation of properties of monovalent systems. Direct extension of this method to even divalent systems faces two major problems.

First, use of the Rayleigh-Schrödinger RMBPT for heavy systems with more than one valence electron leads to a non-symmetric effective Hamiltonian and to the 
problem of "intruder states" [146]. Second, the complexity of the all-order formalism for matrix elements increases rapidly with the number of valence electrons. The direct extensions of the all-order approach to more complicated systems is impractical. For example, the expression for all-order matrix elements in divalent systems contains several hundred terms instead of the twenty terms in the corresponding monovalent expression. However, combining the linearized coupled-cluster approach (also referred to as the all-order method) with CI method eliminates many of these difficulties. This method (referred to as CI+all-order) was developed in Ref. [139] and tested on the calculation of energy levels of $\mathrm{Mg}, \mathrm{Ca}, \mathrm{Sr}, \mathrm{Zn}, \mathrm{Cd}$, $\mathrm{Ba}$, and $\mathrm{Hg}$. The prefix $\mathrm{R}$ is used to indicate the use of the relativistic Hamiltonian.

In the RCI+all-order approach, the effective Hamiltonian is constructed using fully converged all-order excitations coefficients $\rho_{m a}, \rho_{m n a b}, \rho_{m v}, \rho_{m n v a}$, and $\rho_{m n v w}$ (see section IVF for designations). The $\rho_{\text {mnvw }}$ coefficients do not arise in the monovalent all-order method, but are straightforwardly obtained from the above core and core-valence coefficients. As a result, the core-core and core-valence sectors of the correlation corrections for systems with few valence electrons are treated with the same accuracy as in the all-order approach for the monovalent systems. The CI method is used to treat valencevalence correlations and to evaluate matrix elements and polarizabilities.

The RCI+all-order method employs a variant of the Brillouin-Wigner many-body perturbation theory, rather than Rayleigh-Schrödinger perturbation theory. In the Brillouin-Wigner variant of MBPT, the effective Hamiltonian is symmetric and accidentally small denominators do not arise [139]. Comparisons of the RCI+MBPT and RCI+all-order binding energies for the ground and excited states of a number of two-electron systems reveal that the RCI+all-order energies are usually more accurate by at least a factor of three [139].

The preliminary calculations of polarizabilities values in $\mathrm{Ca}$ and $\mathrm{Sr}$ indicate better agreement of the RCI+allorder $a b$ initio results with recommended values from Ref. [13] in comparison with the RCI+MBPT approach.

\section{BENCHMARK COMPARISONS OF THEORY AND EXPERIMENT}

\section{A. Noble gases and isoelectronic ions}

Theoretical [36, 50, 104, 116, 117, 147, 148, 150, 151, 158, 280] and experimental 61, 62, 112, 152 155, 159 167] values for the ground state polarizabilities of the noble gases and isoelectronic ions are listed in Table IV References are given in square brackets. The reference is given at the end of the row when all data in this row come from the same work. Otherwise, the references are listed together with the particular value. The following method abbreviations are used in the table: DC - dielec- tric constant, RI - refractive index, SA - spectral analysis, RRPA - relativistic random phase approximation, MBPT - many-body perturbation theory, (R)CCSDT - (relativistic) coupled-cluster method. If any triple excitations are included, CCSDT abbreviation is used for coupledcluster calculations, single-double coupled cluster calculations are labelled (R)CCSD. The RCCR12 calculation 151] is a CCSDT calculation which allows for explicitly correlated electron pairs. The pseudo-natural orbital coupled electron pair approximation (PNO-CEPA) 104] can be regarded as precursor of modern CCSD type models. We first discuss the general trends of values for the noble gases as a whole, and then consider $\mathrm{He}$ in more detail separately.

The most precise calculations of the noble gas polarizabilities (apart from helium) have mostly been obtained with coupled-cluster type calculations. As we noted in the previous sections, particular care has to be taken to ensure that the basis set used in CC calculations is of sufficiently high quality to obtain accurate values. One curious aspect about the noble gases is their insensitivity to relativistic effects. The relativistic correction to $\alpha_{0}$ is less than $1 \%$ for $\mathrm{Ne}, \mathrm{Ar}$, and $\mathrm{Kr}$ and is only about $2 \%$ for Xe [148].

One notable feature of Table IV is the good agreement of the RRPA [50] with the much more elaborate coupled-cluster and Hylleraas basis function calculations and experimental data. The difference between RRPA values and other calculations/experimental value for neutral systems ranges from $10 \%$ for Ne to $1.6 \%$ for $\operatorname{Kr}(4 \%$ for $\mathrm{He}$ ). The RRPA values [50] improve significantly for the singly ionized systems and differ from other values by $5 \%$ for $\mathrm{Na}^{+}$and only $0.4 \%$ for $\mathrm{Rb}^{+}$. The discrepancies are reduced further for doubly ionized systems owing to the decrease in the relative contribution of the correlation corrections beyond RRPA. Core polarizabilities for the alkali and alkaline-earth atoms are important for the construction of CICP type models of these atoms. In addition, the RRPA calculations of the core polarizabilities are embedded into many calculations of the polarizabilities of alkali and alkaline-earth ions (see, for example, Refs. [13, 56, 57]).

\section{Helium}

The helium atom is of particular interest since it allows for the most precise calculations and benchmark tests of theory and experiment. Within the framework of the non-relativistic Schrödinger equation with infinite-nuclear-mass Hamiltonian, the He polarizability value obtained using a modified version of the generalized Hylleraas basis set 281] is 1.383192174455(1) a.u. [116], achieving accuracy of 13 significant digits. This value is in agreement with 1996 calculation of [117].

The finite mass effects increase the polarizability by about 0.00062 a.u., with the mass polarization effect accounting for 0.000049 a.u. resulting in the ${ }^{4} \mathrm{He}$ nonrel- 
TABLE IV: Ground state polarizabilities $\alpha_{0}$ (in atomic units) of noble gases and isoelectronic ions. Uncertainties in the last digits are given in parentheses. References are given in square brackets. Method abbreviations: DC - dielectric constant, RI refractive index, SA - spectral analysis, RRPA - relativistic random-phase approximation, (R)CCSDT - (relativistic) coupledcluster calculations. The RCCR12 calculation is a CCSDT calculation which allows for explicitly correlated electron pairs. ${ }^{a}$ See text for further discussion of He polarizability calculations, ${ }^{b}$ Finite mass Hylleraas alculation incorporating relativistic effects from an RCI calculation as an additive correction, ${ }^{c}$ PNO-CEPA (pseudo-natural orbital coupled electron pair approximation).

\begin{tabular}{|c|c|c|c|c|c|c|}
\hline $\mathrm{He}$ & $\mathrm{Ne}$ & $\mathrm{Ar}$ & $\mathbf{K r}$ & $\mathrm{Xe}$ & $\mathbf{R n}$ & Method [Ref.] \\
\hline 1.322 & 2.38 & 10.77 & 16.47 & 26.97 & & Th. RRPA [50] \\
\hline \multirow[t]{5}{*}{1.383763} & 2.6648 & 11.084 & & & & Th. CCSDT [147] \\
\hline & 2.697 & 11.22 & 16.80 & 27.06 & 33.18 & Th. RCCSDT [148] \\
\hline & $2.665[149]$ & $11.085(6)[36]$ & & & & Th. CCSDT \\
\hline & 2.6557 & 11.062 & 17.214 & 28.223 & & Th. MBPT [150] \\
\hline & $2.668(6)[151]$ & & & & & Th. RCCR12 \\
\hline $1.38376079(23)^{a, b}[119]$ & & & & & & Th. \\
\hline $1.383223(67)[152,153]$ & $2.670(3)[154]$ & $11.081(5)[154]$ & $16.766(8)[154]$ & & & Expt. DC \\
\hline 1.3838 & 2.6680 & 11.091 & 16.740 & 27.340 & & Expt. RI [62] \\
\hline 1.384 & 2.663 & 11.080 & 16.734 & 27.292 & & Expt. RI [61] \\
\hline $1.383759(13)[63]$ & & $11.083(2)[155]$ & & & & Expt. RI \\
\hline $\mathbf{L i}^{+}$ & $\mathrm{Na}^{+}$ & $\mathbf{K}^{+}$ & $\mathbf{R b}^{+}$ & $\mathrm{Cs}^{+}$ & $\mathrm{Fr}^{+}$ & \\
\hline $0.192486^{b}[156,157]$ & $0.9947^{c}[104]$ & $5.354^{c}[104]$ & & & & Th. \\
\hline \multirow[t]{2}{*}{0.1894} & 0.9457 & 5.457 & 9.076 & 15.81 & & Th. RRPA [50] \\
\hline & $1.00(4)$ & $5.52(4)$ & $9.11(4)$ & $15.8(1)$ & $20.4(2)$ & Th. RCCSDT [158] \\
\hline \multirow[t]{3}{*}{$0.1883(20)[159]$} & $0.978(10)[160]$ & $5.47(5)[160]$ & $9.0[161]$ & $15.544(30)[162]$ & & Expt. SA \\
\hline & $1.0015(15)[163]$ & & & $15.759[164]$ & & Expt. SA \\
\hline & $0.9980(33)[165]$ & & & $15.644(5)[112,166]$ & & Expt. SA \\
\hline $\mathrm{Be}^{2+}$ & $\mathrm{Mg}^{2+}$ & $\mathbf{C a}^{2+}$ & $\mathrm{Sr}^{2+}$ & $\mathrm{Ba}^{2+}$ & $\mathbf{R a}^{2+}$ & \\
\hline 0.05182 & 0.4698 & 3.254 & 5.813 & 10.61 & & Th. RRPA [50] \\
\hline \multirow[t]{4}{*}{$0.052264^{b}[156,157]$} & $0.4814^{c}[104]$ & $3.161^{c}[104]$ & & & & Th. \\
\hline & & 3.262 & 5.792 & 10.491 & 13.361 & Th. RCCSDT [35] \\
\hline & $0.489(5)[160]$ & $3.26(3)[160]$ & & & & Expt. SA \\
\hline & $0.486(7)[167]$ & & & & & Expt. SA \\
\hline
\end{tabular}

ativistic value of 1.38380999 a.u. [116, 119, 282]. The $\alpha^{2}$ relativistic corrections contribute $-0.00008035(2)$ a.u. [116, 119, 282]. The $\alpha^{3}$ QED corrections with exception of the terms containing electric-field derivative of the Bethe logarithm were calculated in Ref. [116] to give 0.0000305 a.u.. These latter terms were calculated in [119], together with the estimates of the $\alpha^{4}, \alpha^{2} m_{e} / M_{\mathrm{He}}$, and $\alpha^{3} m_{e} / M_{\mathrm{He}}$, yielding the final value of ${ }^{4} \mathrm{He}$ polarizability of $1.38376079(23)$ a.u. listed in Table IV

A non-relativistic coupled-cluster calculation in the infinite mass limit carried out in Ref. [147] provides a detailed study of the dependence of the CCSDT results on the choice of the basis set and tests of basis set convergence. The values obtained with different uncontracted, even-tempered basis sets varied in the fifth significant digit. Their final value of $\alpha\left({ }^{\infty} \mathrm{He}\right)=1.383763$ a.u. differs from the exact non-relativistic Hylleraas value of 1.383192 a.u. [116, 117] at the same level.

A microwave cavity was recently used to measure the refractive index of helium giving a polarizability of 1.383759(13) a.u. [63]. The best experiment has an un- certainty of about $10 \mathrm{ppm}$ and is in accord with the most accurate theory value [119]. Availability of such precise theoretical and experimental values of He polarizability allows for accurate determinations of the thermodynamic temperature and may lead to a more accurate value of the Boltzmann constant [63]. This application is discussed in more detail in Section VIIE.

\section{B. Monovalent systems}

The theoretical 30, 35, 56, 84, 96, 105, 117, 132, 168, 169, 171-178, 180, 188-193, 283 and experimental 67, 71 75, 90, 181, 183-186, 194-199, 284 values of static scalar $\left(\alpha_{0}\right)$ and tensor $\left(\alpha_{2}\right)$ polarizabilities of alkali atoms and scalar static polarizabilities of singly ionized monovalent ions are compared in Table $\mathrm{V}$ and VI. The same designations are used as in the noble gas table. The following additional method abbreviations are used: EH - $E-H$ balance or beam-deflection, sum-rule hybrid $f$-sum rules with experimental data for primary 
TABLE V: Ground and $n p_{j}$ excited state polarizabilities (in a.u.) of alkali atoms. Scalar $\left(\alpha_{0}\right)$ and tensor $\left(\alpha_{2}\right)$ polarizabilities are given for the $n p_{3 / 2}$ states. Static polarizabilities for the $n p_{1 / 2}$ and $n p_{3 / 2}$ states are the same for the non-relativistic Hylleraas and CICP calculations. Uncertainties in the last digits are given in parentheses. References are given in square brackets. Method abbreviations: EH - $E$ - $H$ balance or beam-deflection, sum-rule - hybrid $f$-sum rules with experimental data for primary contribution, SA - spectral analysis, CI - configuration interaction, CICP - CI calculations with a semi-empirical core potential, MBPT - many-body perturbation theory, RLCCSDT - linearized CCSD method with partial triple contributions. All values in the sum-rule row explicitly include a core polarizability. ${ }^{a}$ Non-relativistic Hylleraas calculation for ${ }^{\infty} \mathrm{Li},{ }^{b} \mathrm{Hylleraas}$ calculations for ${ }^{7} \mathrm{Li}$ that includes estimate of relativistic effects, ${ }^{c} \mathrm{CI},{ }^{d}$ Hybrid-RLCCSD data for the alkali ground states from [56] are listed as recommended "sum-rule" data, ${ }^{e}$ interferometry, ${ }^{f}$ interferometry ratio, ${ }^{g}$ cold atom velocity change experiments.

\begin{tabular}{|c|c|c|c|c|c|c|c|}
\hline & $\mathrm{Li}$ & $\mathrm{Na}$ & $\mathbf{K}$ & $\mathbf{R b}$ & Cs & $\mathrm{Fr}$ & Method \\
\hline \multirow{11}{*}{$\alpha_{0}$} & $2 s$ & $3 s$ & $4 s$ & $5 s$ & $6 s$ & $7 s$ & \\
\hline & $164.112(1)^{a}[118]$ & $164.50^{c}[168]$ & & & $398.2(9)^{* *}[169]$ & & Th. \\
\hline & $164.11(3)^{b}[170]$ & & & & & & Th. Hyl. \\
\hline & $164.21[171]$ & 162.8 [105] & 290.0 [105] & 315.7 [105] & & & Th. CICP \\
\hline & & $165.50[172]$ & 301.28 [173] & & & & Th. CCSD \\
\hline & 163.74 [173] & $162.9(6)[174]$ & 291.12 [132] & 316.17 [132] & 396.02 [132] & 315.23 [132] & Th. RCCSDT \\
\hline & & 163.0 & 289.1 & 316.4 & 401.5 & 315.1 & Th. RLCCSD [56] \\
\hline & $164.08[175]$ & & $289.3[176]$ & & $398.4(7)[177]$ & 313.7 [178] & Th. RLCCSDT \\
\hline & $164(3)$ & $159(3)$ & $293(6)$ & $319(6)$ & $402(8)$ & & Expt. EH [67] \\
\hline & $164.2(1.1)^{e}[71]$ & $162.7(8)^{e}[72]$ & $290.8(1.4)^{f}[73]$ & $318.8(1.4)^{f}[73]$ & $401.0(6)^{g}$ & & Expt. \\
\hline & & $162.6(3)$ & $290.2(8)$ & $318.6(6)$ & $399.9(1.9)$ & $317.8(2.4)$ & Sum-rule $^{d}[56]$ \\
\hline \multirow{5}{*}{$\alpha_{0}$} & $2 p_{1 / 2}$ & $3 p_{1 / 2}$ & $4 p_{1 / 2}$ & $5 p_{1 / 2}$ & $6 p_{1 / 2}$ & & \\
\hline & $126.9458(3)[118]$ & & & & & & Th. ${ }^{a} \mathrm{Hyl}$. \\
\hline & $126.95[171]$ & 360.7 [179] & 615.3 [179] & 854.4 [179] & & & Th. CICP \\
\hline & $126.980[175]$ & & $604.1[176]$ & $805(31) \quad[30]$ & 1338(54) [177] & & Th. RLCCSDT \\
\hline & & 359.7 & 605 & 807 & & & Th. RCI+MBPT [180] \\
\hline \multirow[t]{3}{*}{$\alpha_{0}$} & $2 p_{3 / 2}$ & $3 p_{3 / 2}$ & $4 p_{3 / 2}$ & $5 p_{3 / 2}$ & $6 p_{3 / 2}$ & & \\
\hline & $126.995[175]$ & & $614.1[176]$ & & 1648(58) [177] & & Th. RLCCSDT \\
\hline & & 361.4 & 616 & 870 & & & Th. RCI+MBPT [180] \\
\hline \multirow{7}{*}{$\alpha_{2}$} & $2 p_{3 / 2}$ & $3 p_{3 / 2}$ & $4 p_{3 / 2}$ & $5 p_{3 / 2}$ & $6 p_{3 / 2}$ & & \\
\hline & $1.6214(3)[118]$ & & & & & & Th. Hyl. \\
\hline & $1.6627[171]$ & $-87.89[179]$ & $-107.9[179]$ & $-160.5[179]$ & & & Th. CICP \\
\hline & & -88.0 & -111 & -171 & & & Th. RCI+MBPT [180] \\
\hline & $1.59[175]$ & & $-107.9[176]$ & & $-261(13)[177]$ & & Th. RLCCSDT \\
\hline & $1.64(4)[181]$ & $-88.3(4)[182]$ & $-107(2)[183]$ & $-163(3)[183]$ & $-261(8)[90]$ & & Expt. \\
\hline & & $-113(16)[184]$ & $-110.9(2.8)[185]$ & & $-262.4(1.5)[186]$ & & Expt. \\
\hline
\end{tabular}

contribution, RESIS - resonant excitation Stark ionization spectroscopy, RLCCSDT - linearized CCSD method with partial triple contributions included. First, some general remarks are made for monovalent systems, and then $\mathrm{Li}, \mathrm{Na}, \mathrm{Mg}^{+}$and $\mathrm{Cs}$ are considered in more detail.

The comparatively simple electronic structure of these atoms render them amenable to accurate calculation by the coupled-cluster and CICP methods. The sum-rule polarizabilities 56] come from a hybrid calculation that use the RLCCSD calculation as a template. However, the matrix element for the resonance transition has been replaced by high accuracy experimental matrix elements compiled in [31]. The ab initio RLCCSD values are in excellent agreement (better than 1\%) with these hybrid recommended values. The semi-empirical CICP calculations reveal a similar level of accuracy, although there has been some degradation in accuracy for the heavier $\mathrm{Rb}$ system. The CI calculations with a semi-empirical core potential (CICP) are in excellent agreement with RLCCSDT calculations and experiment for lighter systems. The non-relativistic CICP cannot be expected to be particularly accurate for states with significant spinorbit splitting, e.g. the $n p_{J}$ states of Rb. The best that can be expected is that the CICP calculation will do a reasonable job of reproducing the statistically weighted $n p_{J}$ average polarizability.

The results of the coupled-cluster calculations can be sensitive to particular contributions that are included, owing to cancellations of various terms (for example, some triple excitations beyond perturbative treatment may partially cancel with non-linear single-double terms), leading to some differences between different 
TABLE VI: Ground state polarizabilities (in a.u.) of alkali-like ions. Uncertainties in the last digits are given in parentheses. References are given in square brackets. Method abbreviations: SA - spectral analysis, RESIS - resonant excitation Stark ionization spectroscopy, ${ }^{a}$ non-relativistic Hylleraas calculation for ${ }^{\infty} \mathrm{Be}^{+},{ }^{b}$ Hylleraas calculations for ${ }^{9} \mathrm{Be}^{+}$that includes estimate of relativistic effects, ${ }^{c} f$-sum rule for valence polarizability with core-polarzation from [187] added.

\begin{tabular}{|c|c|c|c|c|c|c|}
\hline $\mathbf{B e}^{+}$ & $\mathrm{Mg}^{+}$ & $\mathrm{Ca}^{+}$ & $\mathbf{S r}^{+}$ & $\mathrm{Ba}^{+}$ & $\mathbf{R} \mathbf{a}^{+}$ & Method \\
\hline $2 s$ & $3 s$ & $4 s$ & $5 s$ & $6 s$ & $7 s$ & \\
\hline $24.4966(1)^{a}[188]$ & & & & & & Th. Hyl. \\
\hline $24.489(4)^{b}[170]$ & & & & & & Th. Hyl. \\
\hline $24.495[189]$ & 35.66 [168] & & & & & Th. CI \\
\hline \multirow[t]{7}{*}{$24.493[188$} & $34.99[84]$ & 75.49 [190] & 89.9 [191] & & & Th. CICP \\
\hline & $35.05[84]$ & $76.1(1.1)[192]$ & $91.3(9)[193]$ & $124.15[96]$ & $106.5[178]$ & Th. RLCCSD \\
\hline & & 75.88 & 91.10 & 123.07 & 105.37 & Th. RCCSDT [35] \\
\hline & $33.80(50)[194]$ & $75.3[195]$ & & $125.5(1.0)[196]$ & & Expt. SA \\
\hline & $35.04(3)[84]$ & & & $124.30(16)[197]$ & & Expt. RESIS \\
\hline & $35.00(5)$ [198] & & & $123.88(5)[199]$ & & Expt. RESIS \\
\hline & $35.10[200]$ & $74.11[200]$ & & & & ${ }^{c} f$-sum rule \\
\hline
\end{tabular}

TABLE VII: Polarizability differences $\alpha_{0}\left(n p_{J}\right)-\alpha_{0}(n s)$ (in a.u.) of the alkali atoms derived from Stark shift measurements. Values are negative when the $n p_{J}$ state polarizability is smaller than the ground state polarizability. Stark shifts for the $n p_{1 / 2}$ and $n p_{3 / 2}$ states are the same for the non-relativistic Hylleraas and CICP methods. Uncertainties in the last digits are given in parentheses. References are given in square brackets. The experimental values and Hylleraas calculations [170] are those reported for ${ }^{7} \mathrm{Li}$, the CICP and RLCCSDT values are for ${ }^{\infty} \mathrm{Li}$.

\begin{tabular}{|c|c|c|c|c|c|}
\hline${ }^{7} \mathbf{L i}$ & $\mathrm{Na}$ & $\mathbf{K}$ & $\mathbf{R b}$ & Cs & Method \\
\hline $2 s-2 p_{1 / 2}$ & $3 s-3 p_{1 / 2}$ & $4 s-4 p_{1 / 2}$ & $5 s-5 p_{1 / 2}$ & $6 s-6 p_{1 / 2}$ & \\
\hline$-37.14(3)[170]$ & & & & & Th. Hylleraas \\
\hline$-37.26[105,171]$ & $197.9[105,179]$ & $325.3[105,179]$ & & & Th. CICP \\
\hline$-37.104[175]$ & $196.7[56,180]$ & $314.8[176]$ & $488(4)^{b}[30,180]$ & $940(55)^{b}[177]$ & Th. RLCCSDT \\
\hline$-37.146(17)[91]$ & & $316.68(4)$ [201] & $491.52(6)[201]$ & $926.08(12) \quad[92]$ & Expt. \\
\hline$-37.11(33)[181]$ & $196.86(45)[202]$ & $315(3)[183]$ & & & Expt. \\
\hline $2 s-2 p_{3 / 2}$ & $3 s-3 p_{3 / 2}$ & $4 s-4 p_{3 / 2}$ & $5 s-5 p_{3 / 2}$ & $6 s-6 p_{3 / 2}$ & \\
\hline$-37.089 \mathrm{MBPT}[175]$ & $198.4[56,180]$ & $324.8[176]$ & $554[56,180]$ & 1250(59) [177] & Th. RLCCSDT \\
\hline \multirow[t]{2}{*}{$-37.30(42)[181]$} & $198.0(6)[182]$ & $322.3(3.2)$ [183] & $538.5(3.2)[183]$ & $1240.2(2.4)[186]$ & Expt. \\
\hline & & & & $1264(13)[90]$ & Expt. \\
\hline
\end{tabular}

coupled-cluster calculations 285]. The properties involving $n d$ states (i.e. $n p$ polarizabilities) are also sensitive to the number of partial of waves included in the basis sets. Omission or inadequate inclusion of partial waves with $l>3$ may lead to poor results for matrix elements involving $n d$ states, and, subsequently, relevant excitedstate polarizabilities.

Some of the most stringent tests of polarizability calculations of monovalent systems come from Stark shift measurements of alkali resonance transitions. Therefore, it is useful to compare the experimental values for the polarizability difference obtained from Stark shift measurement directly with theoretical predictions in these cases. Scalar polarizability differences $\alpha_{0}\left(n p_{J}\right)-\alpha_{0}(n s)$ (in a.u.) of the alkali transitions derived from Stark shift measurements are compared with theoretical values in Table VII 30, 56, 90 92, 105, 170, 171, 175 177, 180, 180183, 186, 201, 202]. For the elements heavier that Li, the finite mass effects are smaller than the uncertainty of the calculation.

The tensor polarizability of an open shell atom can be extracted from the difference in polarizabilities between the different magnetic sub-levels. The scalar and tensor polarizabilities [118, 171, 191-193, 203 206, 208, 208, 209] of some low lying excited states of $\mathrm{Li}, \mathrm{Na}, \mathrm{K}, \mathrm{Rb}$, $\mathrm{Ca}^{+}$and $\mathrm{Sr}^{+}$are listed in Table VIII. There is a paucity of experimental data for excited states, even for wellstudied alkali atoms. The polarizabilities of the $n d_{5 / 2}$ states of $\mathrm{Ca}^{+}$and $\mathrm{Sr}^{+}$are given owing to their importance for evaluation of the black-body radiation shifts. Some older and less accurate Stark shifts and tensor polarizabilities are omitted from these Tables. 
TABLE VIII: Excited state scalar $\alpha_{0}$ and tensor $\alpha_{2}$ polarizabilities (in a.u.) of monovalent systems. All experimental values are derived from Stark shift experiments and the polarizability of the lower state is added to the Stark shift to get the upper state polarizability. Uncertainties in the last digits are given in parentheses. References are given in square brackets. ${ }^{a}$ Hylleraas basis functions, ${ }^{b} \mathrm{CICP},{ }^{c} \mathrm{RLCCSDT},{ }^{d} \mathrm{CA}$. Polarizabilities marked with an asterisk $\left(^{*}\right)$ were not published, but obtained from the matrix elements of [179].

\begin{tabular}{|c|c|c|c|c|}
\hline Atom & State & & Expt. & Theory \\
\hline \multirow[t]{8}{*}{$\mathbf{L i}$} & $3 d_{3 / 2}$ & $\alpha_{0}$ & $-15082(60)$ [203] & $-14928^{a}[118]$ \\
\hline & & & & $-15044^{b}$ \\
\hline & & $\alpha_{2}$ & $11626(68)[203]$ & $11409^{a}$ \\
\hline & & & & $11490^{b}[171]$ \\
\hline & $3 d_{5 / 2}$ & $\alpha_{0}$ & $-15159(32)[203]$ & $-14928^{a}[118]$ \\
\hline & & & & $-15044^{b}[171]$ \\
\hline & & $\alpha_{2}$ & $16308(52)[203]$ & $16298^{a}[118]$ \\
\hline & & & & $16414^{b}$ \\
\hline \multirow[t]{5}{*}{$\mathrm{Na}$} & $5 s_{1 / 2}$ & $\alpha_{0}$ & 21000(1200) [204] & $21780^{* b}[179]$ \\
\hline & $4 d_{3 / 2}$ & $\alpha_{0}$ & $624000(7000)$ [204] & $633800^{* b}$ \\
\hline & & $\alpha_{2}$ & $-154700(2800)[204]$ & $-148700^{* b}[179]$ \\
\hline & $4 d_{5 / 2}$ & $\alpha_{0}$ & $627000(5000)[204]$ & \\
\hline & & $\alpha_{2}$ & $-213800(2000)[204]$ & $-212400^{* b}[179]$ \\
\hline \multirow[t]{2}{*}{$\mathbf{K}$} & $5 p_{3 / 2}$ & $\alpha_{0}$ & & $7118^{* b}$ \\
\hline & & $\alpha_{2}$ & $-1057(161)[205]$ & $-1019^{* b}[179$ \\
\hline \multirow[t]{4}{*}{$\mathrm{Ca}^{+}$} & $3 d_{5 / 2}$ & $\alpha_{0}$ & & $32.73^{b} \quad[190]$ \\
\hline & & & & $32.0(1.1)^{c} \quad[192]$ \\
\hline & $3 d_{5 / 2}$ & $\alpha_{2}$ & & $-25.20^{b}[190]$ \\
\hline & & & & $-24.5(4)^{c}[192]$ \\
\hline \multirow[t]{4}{*}{$\mathbf{R b}$} & $6 p_{3 / 2}$ & $\alpha_{2}$ & $-2090(80)[206]$ & $-2040^{d}[207]$ \\
\hline & $6 d_{3 / 2}$ & $\alpha_{2}$ & $-42.2(28)[208]$ & $-559^{d}[207]$ \\
\hline & $6 d_{5 / 2}$ & $\alpha_{2}$ & $3780(200)$ [208] & $3450^{d}$ \\
\hline & $7 p_{3 / 2}$ & $\alpha_{2}$ & $-12900(800)$ [209] & $-12500^{d}[207]$ \\
\hline \multirow[t]{4}{*}{$\mathrm{Sr}^{+}$} & $4 d_{5 / 2}$ & $\alpha_{0}$ & & $61.77^{a}$ \\
\hline & & & & $62.0(5)^{c}[193]$ \\
\hline & $4 d_{5 / 2}$ & $\alpha_{2}$ & & $-47.20^{a}[191]$ \\
\hline & & & & $-47.7(3)^{c}[193]$ \\
\hline
\end{tabular}

\section{Lithium}

The lithium polarizability could assume a pivotal role in polarizability metrology if a multi-species interferometer can be constructed that is capable of measuring the ratio of the polarizability of other atoms to that of $\mathrm{Li}$ to a relative accuracy of $10^{-4}$ [70]. In this case, a measurement of such ratios in conjunction with a definitive calculation of the Li $\alpha_{0}$ could lead to new accuracy benchmarks for the polarizabilities of a number of elements.

Correlated basis calculations are possible for lithium since it only has three electrons. Consequently it has
TABLE IX: Selected theoretical and experimental ground state polarizabilities $\alpha_{0}$ (in a.u.) of sodium atom. Uncertainties in the last digits are given in parentheses. References are given in square brackets. HF - Hartree-Fock, PNO-CEPA - pseudonatural orbital configuration expansion, CICP - CI calculations with a semi-empirical core potential, RLCCSDT - linearized CCSD method with additional partial triple contributions included, EH - $E-H$ balance or beam-deflection.

\begin{tabular}{lcc}
\hline \hline Method & Year & Value \\
\hline & Theory & \\
HF [210] & 1964 & 183 \\
HF [104] & 1984 & 189.2 \\
PNO-CEPA [211] & 1976 & 165.02 \\
CICP [212] & 1979 & 162.6 \\
CICP [104] & 1984 & 162.4 \\
CICP [105] & 2003 & 162.8 \\
CI [168] & 2007 & 164.50 \\
RLCCSD [56] & 1999 & 163.0 \\
RCCSDT [173] & 1999 & 164.89 \\
CCSDT [213] & 2001 & 165.06 \\
RCCSDT [214] & 2003 & 166.3 \\
RCCSDT [172] & 2004 & 165.5 \\
CCSDT [174] & 2005 & $162.88(60)$ \\
& 1959 & \\
$f$-sum [215] & 1974 & 166 \\
EH [66] & 1974 & $165(11)$ \\
EH [67] & 1995 & $162.7(8)$ \\
Interferometry [72] & 1999 & $162.6(3)$ \\
Hybrid f-sum [56] & 2010 & \\
Interferometry [73] & & \\
\hline \hline
\end{tabular}

been possible to calculate the polarizability to very high precision [117, 118]. The uncertainty in the experimental value of the polarizability $164.2(11)$ a.u. 71$]$ spans all of the theoretical results reported in Table $\mathrm{V}$

The most recent Hylleraas calculation gave $\alpha_{0}=$ 164.112(1) a.u. for ${ }^{\infty} \mathrm{Li}$ [118]. Including finite mass effects gave $\alpha_{0}=164.161(1)$ a.u. for ${ }^{7} \mathrm{Li}$. An approximate treatment of relativistic effects gave a recommended value of 164.11(3) a.u. 170]. Hylleraas polarizabilities could also serve as benchmarks for coupled-cluster type calculations which can be applied to atoms heavier than lithium.

The most stringent test of Li polarizability calculations is presently the Stark shift measurement of the $2 s-2 p_{1 / 2}$ transitions by Hunter et al. 91], which gave a polarizability difference of $-37.14(2)$ a.u. The current theoretical benchmark is the recent Hylleraas calculations that include finite mass and relativistic effects [118, 170]. The ${ }^{7} \mathrm{Li}$ Hylleraas polarizability difference of $-37.14(4)$ a.u. [170] is in excellent agreement with the experimental polarizability difference 91]. The RLCCSDT value of -37.104 is within 2 standard deviations of the Hunter experiment while the CICP value is 4 standard deviations 
TABLE X: Excited state scalar $\alpha_{0}$ and tensor $\alpha_{2}$ polarizabilities (in multiples of 1000 a.u.) of the Cs atom. Uncertainties in the last digits are given in parentheses. References are given in square brackets. Experimental values: ${ }^{b}$ derived from the Ref. [93 $7 s-6 s$ Stark shift measurement and the $6 s$ result from [74], ${ }^{c}$ Ref. [216], ${ }^{d}$ Refs. [217, 218], ${ }^{e}$ Ref. 219], ${ }^{f}$ Ref. [220], ${ }^{g}$ Ref. 221], ${ }^{h}$ Ref. [222], ${ }^{i}$ Ref. 223], CA - Coulomb approximation, RLCCSDT - relativistic linearized coupled-cluster method with single, double, and partial triple excitations.

\begin{tabular}{|c|c|c|c|c|c|c|c|}
\hline$\alpha_{0}$ & $7 s$ & $8 s$ & $9 s$ & $10 s$ & $11 s$ & $12 s$ & Ref. \\
\hline & $6.238(41)$ & $38.27(28)$ & $153.7(1.0)$ & $478(3)$ & $1246(8)$ & $2866(30)$ & Th. RLCCSDT [177] \\
\hline & 6.14 & 37.9 & 153 & 475 & 1240 & 2840 & Th. CA [218] \\
\hline & $6.238(6)^{b}$ & $38.06(25)^{c}$ & & $478.5(1.1)^{d}$ & $1245(1)^{d}$ & $2867(2)^{d}$ & Expt. \\
\hline \multirow[t]{5}{*}{$\alpha_{0}$} & $7 p_{1 / 2}$ & $8 p_{1 / 2}$ & $9 p_{1 / 2}$ & $10 p_{1 / 2}$ & & & \\
\hline & $29.9(7)$ & $223(2)$ & $1021(7)$ & $3499(19)$ & & & Th. RLCCSDT [177] \\
\hline & 29.4 & 221 & 1020 & 3490 & & & Th. CA [218] \\
\hline & $29.6(6)$ & & & & & & Expt. [224] \\
\hline & $7 p_{3 / 2}$ & $8 p_{3 / 2}$ & $9 p_{3 / 2}$ & $10 p_{3 / 2}$ & & & \\
\hline \multirow[t]{3}{*}{$\alpha_{0}$} & $37.5(8)$ & $284(3)$ & $1312(7)$ & $4522(19)$ & & & Th. RLCCSDT [177] \\
\hline & 36.9 & 282 & 1310 & 4510 & & & Th. CA [218] \\
\hline & $37.9(8)$ & & & & & & Expt. [219] \\
\hline \multirow[t]{6}{*}{$\alpha_{2}$} & $-4.41(17)$ & $-30.6(6)$ & $-135(2)$ & $-451(5)$ & & & Th. RLCCSDT [177] \\
\hline & -4.28 & -30.2 & -134 & -449 & & & Th. CA [218] \\
\hline & $-4.43(12)^{e}$ & $-30.5(1.2)^{f}$ & & & & & Expt. \\
\hline & $-4.33(17)$ & & & & & & Expt. [206] \\
\hline & $-4.00(8)$ & & & & & & Expt. [224] \\
\hline & $5 d_{3 / 2}$ & $6 d_{3 / 2}$ & $7 d_{3 / 2}$ & $8 d_{3 / 2}$ & $9 d_{3 / 2}$ & $10 d_{3 / 2}$ & \\
\hline \multirow[t]{3}{*}{$\alpha_{0}$} & $-0.352(69)$ & $-5.68(45)$ & $-66.7(1.7)$ & $-369(5)$ & $-1402(13)$ & $-4234(32)$ & Th. RLCCSDT $[177,225]$ \\
\hline & -0.418 & -5.32 & -65.2 & -366 & -1400 & -4220 & Th. CA [218] \\
\hline & & & $-60(8)^{g}$ & & $-1450(120)^{f}$ & $-4185(4)^{h}$ & Expt. \\
\hline \multirow[t]{4}{*}{$\alpha_{2}$} & $0.370(28)$ & $8.77(36)$ & $71.1(1.2)$ & $339(4)$ & $1189(10)^{i}$ & $3416(26)$ & Th. RLCCSDT $[177,225]$ \\
\hline & 0.380 & 8.62 & 70.4 & 336 & 1190 & 3410 & Th. CA [218] \\
\hline & & & $74.5(2.0)^{i}$ & $332(16)^{f}$ & $1183(35)^{i}$ & $3401(4)^{h}$ & Expt. \\
\hline & $5 d_{5 / 2}$ & $6 d_{5 / 2}$ & $7 d_{5 / 2}$ & $8 d_{5 / 2}$ & $9 d_{5 / 2}$ & $10 d_{5 / 2}$ & \\
\hline \multirow[t]{3}{*}{$\alpha_{0}$} & $-0.453(70)$ & $-8.37(55)$ & $-88.8(2.0)$ & $-475(5)$ & $-1777(14)$ & $-5316(38)$ & Th. RLCCSDT $[177,225]$ \\
\hline & -0.518 & -7.95 & -87.1 & -472 & -1770 & -5300 & Th. CA [218] \\
\hline & & & $-76(8)^{g}$ & & $-2050(100)^{f}$ & $-5303(8)^{h}$ & Expt. \\
\hline \multirow[t]{4}{*}{$\alpha_{2}$} & $0.691(40)$ & $17.33(50)$ & $142(2)$ & $678(5)$ & $2386(13)$ & $6869(34)$ & Th. RLCCSDT $[177,225]$ \\
\hline & 0.704 & 17.00 & 140 & 675 & 2380 & 6850 & Th. CA [218] \\
\hline & & & $129(4)^{g}$ & $731(40)^{f}$ & $2650(140)^{f}$ & $6815(20)^{h}$ & Expt. \\
\hline & & & & & & $7110(360)$ & Expt. [220] \\
\hline
\end{tabular}

too large. TableVII shows that the Stark shift data offer the most precise information with which to discriminate between various theoretical calculations.

\section{Sodium}

A chronological list detailing selected values [56, 66, 67, 72, 73, 104, 105, 168, 172 174, 210 215] of the sodium ground state polarizability is presented in Table IX. The theory values are also sorted by the type of calculation. The $3 s \rightarrow 3 p$ resonant transition accounts for $98.8 \%$ of the polarizability.

The most notable feature of this table is the excellent agreement of the semi-empirical CICP type calculations with the recent high-precision experimental values of 162.6(3) a.u. [56] and 162.7(8) a.u. [72]. All three calculations [104, 105, 212], performed over a period of three decades lie within the experimental uncertainties.

The coupled-cluster calculations, with the exception 
TABLE XI: Ground and excited $\left(n s n p{ }^{3} P_{0}\right)$ state scalar polarizabilities $\alpha_{0}$ (in a.u.) of group II atoms and divalent ions. Uncertainties in the last digits are given in parentheses. References are given in square brackets. The abbreviations conform to those used in Table IV and V] Hybrid-RCI+MBPT include experimental data for some transitions. ${ }^{a}$ RCCSDT, ${ }^{b} \mathrm{MBPT},{ }^{c} \mathrm{Hybrid}-\mathrm{RCI}+\mathrm{MBPT}$ data for the alkaline-earth ground states from [57] are listed as recommended "sum-rule" data, ${ }^{d} \mathrm{RCI}+\mathrm{MBPT}$.

\begin{tabular}{|c|c|c|c|c|c|c|}
\hline $\begin{array}{l}\mathbf{B e} \\
2 s^{2}\end{array}$ & $\begin{array}{l}\mathrm{Mg} \\
3 s^{2}\end{array}$ & $\begin{array}{l}\mathbf{C a} \\
4 s^{2}\end{array}$ & $\begin{array}{l}\mathbf{S r} \\
5 s^{2}\end{array}$ & $\begin{array}{l}\mathbf{B a} \\
6 s^{2}\end{array}$ & $\begin{array}{l}\mathbf{R a} \\
7 s^{2} \\
\end{array}$ & Method \\
\hline $37.755[121]$ & & & & & & Th. ECG \\
\hline $37.73(5)^{a}[226]$ & $71.7^{b}[227]$ & $157^{b}[227]$ & & & & Th. \\
\hline $37.807[228]$ & $70.90[229]$ & $171.7[230]$ & & & & Th. CI \\
\hline $37.29[104]$ & $70.74[104]$ & $156.0[104]$ & & & & Th. CICP \\
\hline \multirow[t]{3}{*}{37.69 [105] } & $71.35[105]$ & $159.4[105]$ & 201.2 [105] & & & Th. CICP \\
\hline & & $158.00[35]$ & $198.85[35]$ & $273.9[35]$ & $248.56[35]$ & Th. RCCSDT \\
\hline & & $152[133]$ & $190[133]$ & $275.5[134]$ & & Th. RCCSDT \\
\hline \multirow[t]{3}{*}{$37.76[57]$} & $71.33[57]$ & $159.0[57]$ & $202.0[57]$ & $272.1[57]$ & & Th. RCI+MBPT \\
\hline & & $169(17)[68]$ & $186(15)[69]$ & $268(22)[69]$ & & Expt. EH \\
\hline & $74.9(2.7)[231]$ & $157.1(1.3)[57]$ & $197.2(2)[57]$ & $273.5(2.0)[57]$ & & Sum-rule ${ }^{c}$ \\
\hline $2 s 2 p{ }^{3} P_{0}^{o}$ & $3 s 3 p^{3} P_{0}^{o}$ & $4 s 4 p^{3} P_{0}^{o}$ & $5 s 5 p^{3} P_{0}^{o}$ & $6 s 6 p^{3} P_{0}^{o}$ & & \\
\hline \multirow[t]{3}{*}{$39.02[28]$} & $101.5[232,233]$ & $295.3[234]$ & & & & Th. CICP \\
\hline & $101.2(3)[13]$ & $290.3(1.5)[13]$ & $458.3(3.6)[13]$ & & & Th. Hybrid-RCI+MBPT \\
\hline & & & $457.0[141]$ & $-13[53]$ & & Th. RCI+MBPT \\
\hline $\mathrm{Al}^{+}$ & $\mathrm{Si}^{2+}$ & $\mathrm{Zn}$ & $\mathrm{Cd}$ & $\mathrm{Hg}$ & $\mathbf{Y b}$ & \\
\hline $3 s^{2}$ & $3 s^{2}$ & $4 s^{2}$ & $5 s^{2}$ & $5 d^{10} 6 s^{2}$ & $4 d^{14} 6 s^{2}$ & \\
\hline $24.2^{b}[227]$ & & & & & & Th. \\
\hline $24.14(12)[235]$ & $11.688[88]$ & $38.12[113]$ & $44.63[113]$ & 31.32 [113] & & Th. CICP \\
\hline \multirow[t]{8}{*}{24.12 CI [229] } & $11.75 \mathrm{CI}[229]$ & & & $33.6^{d}[145]$ & $111.3^{d}[13]$ & Th. \\
\hline & & & & & $138.9[143]$ & Th. RCI+MBPT \\
\hline & & & & & $141(6)[143]$ & Th. Hybrid-RCI+MBPT \\
\hline & & $39.2(8)[236]$ & & & $140.4[237]$ & Th. RCCSDT \\
\hline & & $38.8(8)[236]$ & 49.65(1.49) [64] & $33.75[238]$ & & Expt. RI \\
\hline & & & & $33.91[65]$ & & Expt. RI \\
\hline & $11.666(4)$ [239] & & & & & Expt. RESIS \\
\hline & $11.669(9)[88]$ & & & & & Expt. RESIS \\
\hline $24.20(75)[231]$ & & & & & & Sum-rule \\
\hline $3 s 3 p^{3} P_{0}^{o}$ & & $4 s 4 p^{3} P_{0}^{o}$ & $5 s 5 p^{3} P_{0}^{o}$ & $6 s 6 p^{3} P_{0}^{o}$ & $6 s 6 p^{3} P_{0}^{o}$ & \\
\hline \multirow[t]{5}{*}{$24.62(25)[235]$} & & 67.69 [113] & 75.29 [113] & $55.32[113]$ & & Th. CICP \\
\hline & & & & $54.6[145]$ & 315.9 [143] & Th. RCI+MBPT \\
\hline & & & & & $252(25)[240]$ & Th. RCI+MBPT \\
\hline & & & & & 266(15) [13] & Th. RCI+MBPT \\
\hline & & & & & $302(14)[143]$ & Th. RCI+MBPT \\
\hline
\end{tabular}

of the RLCCSD one [56], tended to give polarizabilities which were $1-2 \%$ larger than experiment until the most recent RCCSDT calculation of Thakkar and Lupinetti [174] which gave 162.9(6) a.u.. The earlier CCSDT calculations tend to overestimate the polarizability most likely due to basis set issues [172, 173, 213, 214]. The same problem could also be leading to the overestimation of the polarizability by the CI [168] and CEPA-PNO [211] calculations.
By way of contrast, the RLCCSD calculation [56] gave a polarizability of 163.0 a.u. which is in agreement with experiment. We have discussed the differences of the RLCCSD approach from the other coupled-cluster calculation in Section IVF. An important feature here is that this calculation uses a $B$-spline basis which is effectively complete [37, 56]. As we have discussed on the example of He CCSD calculation [147], polarizability coupled-cluster results vary significantly with the choice of the basis set 
TABLE XII: Ground state polarizabilities $\alpha_{0}$ (in a.u.) of other systems. Uncertainties in the last digits are given in parentheses. References are given in square brackets. The average over magnetic projections is given for atoms which do not have a spherically symmetric ground state. ${ }^{a}$ Third-order MBPT, ${ }^{b} \mathrm{RRPA},{ }^{c} \mathrm{RCCSD},{ }^{d} \mathrm{RLCCSD},{ }^{e} \mathrm{CICP},{ }^{f}$ light deflection, ${ }^{g} \mathrm{RCI}+\mathrm{MBPT},{ }^{h} \mathrm{RESIS}$ reanalysis using theoretical estimates of higher order polarization corrections, ${ }^{i}$ spectral analysis, ${ }^{j} \mathrm{CI},{ }^{k} \mathrm{CCSDT},{ }^{l} \mathrm{f}$-sum rule, ${ }^{m} \mathrm{RMBPT}$

\begin{tabular}{|c|c|c|c|c|c|c|}
\hline $\begin{array}{l}\mathbf{C u} \\
4 s\end{array}$ & $\begin{array}{l}\mathbf{A g} \\
5 s\end{array}$ & $\begin{array}{l}\mathbf{A u} \\
6 s\end{array}$ & $\begin{array}{l}\mathbf{Z n}^{+} \\
4 s\end{array}$ & $\begin{array}{l}\mathbf{H g}^{+} \\
6 s\end{array}$ & $\begin{array}{l}\mathbf{Y b}^{+} \\
6 s\end{array}$ & Method \\
\hline $45.0[241]$ & $52.2[241]$ & $35.1[241]$ & & & $62.04^{a}[124]$ & Th. \\
\hline $46.50[242]$ & $52.46[242]$ & 36.06 [242] & $18.84[243]$ & $19.36[243]$ & & Th. RCCSDT \\
\hline \multirow[t]{2}{*}{41.65} & 46.17 & & & & & Th. CICP [244] \\
\hline & & $30(4)^{l}[245]$ & $15.4(5)^{l}[246]$ & & & Expt. \\
\hline$\overline{\mathrm{Al}^{2+}}$ & $\mathrm{Si}^{3+}$ & $\mathbf{P}^{3+}$ & $\mathbf{K r}^{6+}$ & $\mathbf{C u}^{+}$ & $\mathbf{A g}^{+}$ & \\
\hline $3 s$ & $3 s$ & $3 s^{2}$ & $3 d^{10} 4 s^{2}$ & $3 d^{10}$ & $4 d^{10}$ & \\
\hline \multirow[t]{5}{*}{$14.44[168]$} & $7.50[168]$ & $6.73[229]$ & & & & Th. CI \\
\hline & $7.399^{e}[84]$ & & & $5.36^{b}[50]$ & $8.829^{b}[50]$ & Th. \\
\hline & $7.419^{d}[84]$ & & $2.555^{m}$ & $6.57^{c}[247]$ & $9.21^{c}[247]$ & Th. \\
\hline & $7.426(12)[248]$ & & $2.69(4)[249]$ & & & Expt. RESIS \\
\hline & $7.433(25)^{h}[84,248]$ & $6.312(10)^{i}[250]$ & & & & Expt. \\
\hline $\mathrm{Al}$ & $\mathrm{Ga}$ & In & $\mathrm{Tl}$ & & & \\
\hline $3 s^{2} 3 p$ & $4 s^{2} 4 p$ & $5 s^{2} 5 p$ & $6 s^{2} 6 p$ & & & \\
\hline $57.74^{k}[36]$ & & & $49.2^{g}[251]$ & & & Th. \\
\hline \multirow{2}{*}{$59.5^{j}[252]$} & & & & & & Th. \\
\hline & $49.9[253]$ & $61.9[253]$ & $51.6[253]$ & & & Th. RCCSDT \\
\hline $46.2(20)[254]$ & & $68.7(8.1)[255]$ & $51.3(5.4)[256]$ & & & Expt. EH \\
\hline $\mathrm{Si}$ & Sn & $\mathrm{Pb}$ & Ir & $\mathbf{U}$ & & \\
\hline $3 s^{2} 3 p^{2}$ & $5 s^{2} 5 p^{2}$ & $6 s^{2} 6 p^{2}$ & & & & \\
\hline $37.0[252]$ & & & & & & Th. CI \\
\hline $37.17[36]$ & & & & & & Th. CCSD \\
\hline \multirow[t]{2}{*}{37.3 [257] } & $52.9[257]$ & $47.3[257]$ & & & & Th. RCCSDT \\
\hline & $42.4(11.0)[257]$ & $47.1(7.0)[257]$ & $54.0(6.7)[258]$ & $137.0(9.4)^{f}[76]$ & & Expt. EH \\
\hline
\end{tabular}

if it is not sufficiently saturated. In summary, large (effectively complete) basis sets are needed for precision polarizability calculations by a coupled-cluster method.

The relativistic correction to the dipole polarizability is about -1.0 a.u. [174]. The three non-relativistic CICP calculations all lie within $0.5 \%$ of the experimental polarizability. As mentioned earlier, these calculations implicitly include relativistic effects to some extent by tuning the core polarization potential to the experimental binding energies. The RLCCSD calculation uses a relativistic Hamiltonian and intrinsically includes relativistic corrections. The recommended value $162.6(3)$ is based on the RLCCSD calculation with resonant $3 s-3 p_{j}$ transition matrix elements replaced by their experimental values.

The Na polarizability of $162.7(8)$ [72] obtained by interferometry experiment served as the reference polarizability in the determination of the $\mathrm{K}$ and $\mathrm{Rb}$ polarizabilities by the interferometry ratio approach [73]. Table $\mathrm{V}$ shows excellent agreement of these values with the hybrid RLCCSD f-sum polarizabilities of [56].

\section{3. $\mathrm{Mg}^{+}$}

We use $\mathrm{Mg}^{+}$to illustrate the RESIS experimental approach owing to recent advances in that area. Both the potential and the problems of determining the polarizabilities of ions using spectral analysis are evident by contrasting the different values listed for $\mathrm{Mg}^{+}$and $\mathrm{Ba}^{+}$. The original analysis of the RESIS data for $\mathrm{Mg}^{+}$reported a dipole polarizability of $35.00(5)$ a.u. [198]. However, the contributions from the $C_{7}$ and $C_{8}$ terms of Eq. (36) can possibly corrupt the value of $\alpha_{0}$ if they are significant as described in Section [II] A more detailed analysis of the polarization plot which explicitly included the $C_{7}$ and $C_{8}$ terms was subsequently performed in Ref. [84]. This polarization plot is shown in Figure 2. The data points including the explicit subtraction of the $C_{7}$ and $C_{8}$ terms show a higher degree of linearity. The revised analysis resulted in $\alpha_{0}=35.05(3)$ a.u.. This is only $0.15 \%$ larger than the original value and lies within the original error limits.

The treatment of non-adiabatic corrections is a much 
TABLE XIII: Excited state scalar $\alpha_{0}$ and tensor $\alpha_{2}$ polarizabilities (in a.u.) of selected systems. Uncertainties in the last digits are given in parentheses. References are given in square brackets. ${ }^{a} \mathrm{CICP},{ }^{b} \mathrm{RCI}+\mathrm{MBPT},{ }^{c} \mathrm{CCSDT},{ }^{d} \mathrm{RCCSDT}$, ${ }^{e}$ one-electron model potential.

\begin{tabular}{|c|c|c|c|c|}
\hline Atom & State & & Expt. & Theory \\
\hline \multirow[t]{5}{*}{$\mathrm{Ca}$} & $4 s 4 p{ }^{1} P_{1}^{o}$ & $\alpha_{0}$ & & $242.4^{a} \quad[234]$ \\
\hline & & $\alpha_{2}$ & $-54.7(1.2)$ [259] & $-55.54^{a} \quad[234]$ \\
\hline & $4 s 4 p^{3} P_{1}^{o}$ & $\alpha_{2}$ & $12.9(3.2)[260]$ & $14.2^{a}$ \\
\hline & & & $10.54(6)[261]$ & \\
\hline & & & $12.1(8)[261,262]$ & \\
\hline \multirow[t]{4}{*}{$\mathrm{Sr}$} & $5 s 5 p{ }^{1} P_{1}^{o}$ & $\alpha_{2}$ & $-63.1(7.6)[263]$ & \\
\hline & & $\alpha_{2}$ & $-57.55(60)[259]$ & \\
\hline & $5 s 5 p^{3} P_{1}^{o}$ & $\alpha_{0}$ & & $498.8^{b}[141]$ \\
\hline & & $\alpha_{2}$ & $-24.5(3.2)[264]$ & \\
\hline \multirow[t]{5}{*}{$\mathrm{Ba}$} & $6 s 6 p{ }^{1} P_{1}^{o}$ & $\alpha_{0}$ & & $409^{b}[53]$ \\
\hline & & $\alpha_{2}$ & $-43.08(40)$ [259] & $-51^{b}[53]$ \\
\hline & & & $-43.4(1.2)[265]$ & \\
\hline & $6 s 5 d{ }^{1} D_{2}$ & $\alpha_{2}$ & $85.2(2.4)[266]$ & $81^{b}[53]$ \\
\hline & $6 p^{2}{ }^{3} P_{2}$ & $\alpha_{2}$ & $-109.7(4)[267]$ & \\
\hline $\mathrm{Zn}$ & $4 s 4 p^{3} P_{1}^{o}$ & $\alpha_{2}$ & $7.35(32)[268]$ & $6.73^{e}[269]$ \\
\hline \multirow[t]{3}{*}{ Cd } & $5 s 5 p{ }^{3} P_{1}^{o}$ & $\alpha_{2}$ & $7.11(32)$ [268] & $6.30^{e}[269]$ \\
\hline & & & $5.10(24)[270]$ & \\
\hline & & & $5.35(16)[270]$ & \\
\hline \multirow[t]{4}{*}{$\mathbf{H g}$} & $6 s 6 p^{3} P_{1}^{o}$ & $\alpha_{0}$ & & $60.6^{b}[145]$ \\
\hline & & $\alpha_{2}$ & $6.31(24)[206]$ & \\
\hline & & & $6.35(8)[271]$ & \\
\hline & & & $6.34(6)[272]$ & \\
\hline \multirow[t]{2}{*}{ Al } & $3 s^{2} 3 p^{2} P_{3 / 2}^{o}$ & $\alpha_{0}$ & & $57.74^{c}[36]$ \\
\hline & & $\alpha_{2}$ & $-8.15(40)[273]$ & $-8.53^{c}[36]$ \\
\hline \multirow[t]{4}{*}{ Tl } & $6 s^{2} 6 p^{2} P_{3 / 2}^{o}$ & $\alpha_{0}$ & & $81.2^{d}[253]$ \\
\hline & & & & $79.6^{b}[251]$ \\
\hline & & $\alpha_{2}$ & $-24.2(3)[274,275]$ & $-24.56^{d}[253]$ \\
\hline & & & & $-25.0^{b} \quad[251]$ \\
\hline \multirow[t]{5}{*}{$\mathbf{Y b}$} & $6 s 6 p{ }^{1} P_{1}^{o}$ & $\alpha_{0}$ & & $501(200)^{b}[240]$ \\
\hline & & $\alpha_{2}$ & $-57.4(5.6)[276]$ & $-118(60)[240]$ \\
\hline & $6 s 6 p^{3} P_{1}^{o}$ & $\alpha_{0}$ & & $278(15)^{b}[240]$ \\
\hline & & $\alpha_{2}$ & $24.26(84)[277]$ & $24.3(1.5)^{b}[240]$ \\
\hline & & & $23.35(52)[278]$ & \\
\hline $\mathbf{Y b}^{+}$ & $5 d^{2} D_{3 / 2}$ & $\alpha_{2}$ & $-82.5(1.3)[279]$ & \\
\hline
\end{tabular}

more serious issue for the $\mathrm{Ba}^{+}$ground state. Table V shows that subsequent analysis of the RESIS data 197, 199 do not lie within their mutual uncertainties. The most recent analysis of RESIS data gave a polarizability of $123.88(5)$ a.u. [199]. This analysis explicitly included non-adiabatic effects from the low-lying $5 d$ excitation. However, non-adiabatic effects from the $6 s-6 p$ excitation are also significant and need to be included for a RESIS polarizability to be regarded as definitive.

The influence of the non-adiabatic effects in the $C_{7}$ and $C_{8}$ terms of Eq. (36) can be minimized by taking mea-

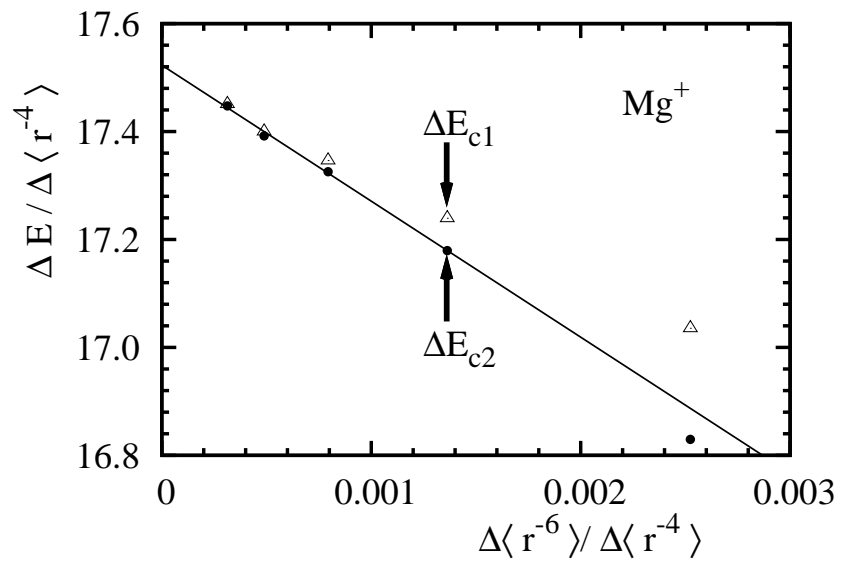

FIG. 2: The polarization plot of the fine-structure intervals of $\mathrm{Mg}$ for the $n=17$ Rydberg levels. The $\Delta E_{c 1}$ intervals are corrected for relativistic, second-order and Stark shifts. The $\Delta E_{c 2}$ intervals account for $\left\langle r^{-7}\right\rangle$ and $\left\langle r^{-8}\right\rangle$ shifts. The linear regression for the $\Delta E_{\mathrm{c} 2}$ plot did not include the last point.

surements at high values of $L$, e.g. $L \geq 8$. Unfortunately, as the non-adiabatic corrections diminish with increasing $L$, the states with very high $L$ are more sensitive to Stark shifts due to stray electric fields. As the energy splitting of the Rydberg states gets smaller at higher $L$, the polarizabilities of the $(n, L)$ levels then get larger due to the very small $(n, L-n, L \pm 1)$ energy differences. To a certain extent one has to choose the $(n, L)$ states to navigate between the low- $L$ Scylla 286] of non-adiabatic corrections and the high- $L$ Charybdis [286] of Stark shifts 84.

\section{Cesium}

The Cs atom has been studied extensively owing to the parity-violation experiments on this system [287]. A comprehensive set of Cs scalar and tensor polarizabilities for the $7 s-12 s, 7 p_{1 / 2}-10 p_{1 / 2}, 7 p_{3 / 2}-10 p_{3 / 2}, 5 d_{3 / 2}-$ $10 d_{3 / 2}$, and $5 d_{5 / 2}-10 d_{5 / 2}$ states [31, 74, 93, 177, 206, 216 225] taken from Ref. [177] is given in Table X.

The polarizabilities listed in Table $\mathrm{X}$ are in $10^{3}$ a.u. since the values range in size from 300 a.u. to $7 \times 10^{6}$ a.u. The results of Ref. [177] are obtained from sumover-state calculation using the RLCCSDT matrix elements and experimental energies for a large number of states. The remaining contributions from highly-excited states were evaluated as well. In a few cases, some of the RLCCSDT matrix elements have been replaced with matrix elements extracted from experiment [177]. Incorporating such highly-excited states as $12 \mathrm{~s}$ required the use of a very large $R=220$ a.u. spherical cavity and large B-spline basis sets. Extensive tests of numerical stability of the calculations in such a large cavity have been conducted to verify the accuracy of a finite basis set representation. All matrix elements used to evaluate 
dominant polarizability contributions were critically evaluated for their accuracy based of the size and type of the dominant correlation corrections and semi-empirical estimates of the omitted correlation terms. Such uncertainty evaluation is discussed in more detail in Section VI.

Coulomb approximation (CA) values [218] were also computed with a sum-over-states approach. One interesting feature of Table $\mathrm{X}$ is the reasonable level of agreement between the CA and RLCCSDT values for many of the polarizabilities. The CA results are computed with wave functions which are tuned to experimental energies. The radial matrix elements that arise in the sum-overstates calculation are dominated by the form of the wave function at large distances. Tuning the wave functions to have the correct energy goes a long way to ensuring that the long-range part of the wave function has the correct shape.

A number of the experimental values in Table $\mathrm{X}$ were obtained from Stark shift experiments. In many cases, the excited state polarizabilities are much larger than the Cs ground state polarizability, so uncertainties in the ground state have minimal impact on the overall uncertainty. The agreement between the experimental and RLCCSDT polarizabilities is excellent for the $n s$ states, in most cases the difference between them is less than $1 \%$. The situation is not so clear-cut for the $n d$ states. Differences between theory and experiment are large in some cases, but so are the uncertainties of many of the experimental values. However, the RLCCSDT results were found in good agreement with more recent experiments [222, 223, 225]. The RLCCSDT calculation 177] provided critically evaluated recommended values for a large number of Cs polarizabilities for which accurate experimental data are not available.

\section{Two electron atoms and ions, $n s^{2}{ }^{1} S$ and nsnp ${ }^{3} P_{0}^{o}$ states}

Table XI gives the polarizabilities for a number of divalent species including the alkaline-earth atoms from Refs. 13, 28, 35, 53, 57, 64, 68, 69, 88, 104, 105, 113, 121, 133, 134, 141, 143, 145, 226 236, 236 240, 299, 300.' The beryllium atom serves as a theoretical benchmark since a very accurate value has been obtained with a basis of exponentially correlated Gaussians (ECG) 121. The CICP [105] and RCI+MBPT polarizabilities [57] lie within $0.2 \%$ of the ECG basis polarizability.

The sub-1\% agreement between the highest quality theory and experiment that occurred for the alkali atoms is not observed for the alkaline-earth atoms owing to their more complicated atomic structure and resulting mixing of configurations. As we have described in Section IVG, perturbative methods do not work well for strong valence-valence correlations. The hybrid values for $\mathrm{Ca}$ and $\mathrm{Sr}$ based on the RCI+MBPT calculations with the matrix elements for the resonance transitions replaced by values derived from experiments are
TABLE XIV: Static polarizability differences (in a.u.) derived from selected Stark shift measurements. Uncertainties in the last digits are given in parentheses. References are given in square brackets. ${ }^{a} \mathrm{RMBPT},{ }^{b} \mathrm{RLCCSDT},{ }^{c} \mathrm{CICP}$, ${ }^{d} \mathrm{RCI}+\mathrm{MBPT}$.

\begin{tabular}{|c|c|c|c|}
\hline Atom & State & Experiment & Theory \\
\hline \multirow[t]{3}{*}{$\mathrm{Cs}$} & $6 s-7 s$ & $5837(6)[93]$ & $5834^{a}[288]$ \\
\hline & & 5709(19) [289] & \\
\hline & $6 s-8 s$ & $37660(250)[216]$ & $37820(290)^{b} \quad[216]$ \\
\hline \multirow[t]{3}{*}{$\mathrm{Mg}$} & $3 s^{2}-3 s 3 p^{3} P_{1}^{o}$ & & \\
\hline & $m=1$ & 32.1(4.0) [290] & $37.6^{c}[232]$ \\
\hline & $m=0$ & $15.7(4)[291]$ & $16.3^{c}[232]$ \\
\hline \multirow[t]{3}{*}{$\mathbf{C a}$} & $4 s^{2}-4 s 4 p^{3} P_{1}^{o}$ & & \\
\hline & $(m=0)$ & $90.4(13.5)[292]$ & $107.5^{c} \quad[234]$ \\
\hline & & $98.97(33)[293]$ & \\
\hline \multirow[t]{2}{*}{$\mathrm{Ba}$} & $6 s^{2}-6 s 6 p^{1} P_{1}^{o}$ & & \\
\hline & $(m=0)$ & $-229.32(48)[294]$ & $-247^{d}$ \\
\hline \multirow[t]{2}{*}{$\mathbf{Y b}$} & $6 s^{2}-6 s 6 p^{3} P_{1}^{o}$ & & $160(60)^{d}[240]$ \\
\hline & $(m=0)$ & $123.85(38)[278]$ & $110(18)^{d}[240]$ \\
\hline $\mathbf{H g}$ & $6 s^{2}-6 s 6 p{ }^{3} P_{1}^{o}$ & $26.68(48)$ [295] & $26.95^{d}[145]$ \\
\hline Ga & $4 s^{2} 4 p_{3 / 2^{-}} 4 s^{2} 5 s$ & $788(40)[183]$ & \\
\hline \multirow[t]{2}{*}{$\mathbf{T l}$} & $6 s^{2} 6 p_{1 / 2^{-}} 6 s^{2} 7 s$ & $-900(48)[296]$ & $-830^{b}[297]$ \\
\hline & & $-829.7(3.1)[298]$ & \\
\hline Tl & $6 p_{1 / 2^{-}} 7 p_{1 / 2}$ & $-4967(249)[296]$ & $-4866^{b}[297]$ \\
\hline $\mathbf{Y} \mathbf{b}^{+}$ & $6 s-5 d^{2} D_{3 / 2}$ & $-41.8(8.5)$ [279] & \\
\hline
\end{tabular}

respectively $1.1 \%$ and $2.5 \%$ smaller than the ab initio RCI+MBPT estimates [57]. With the exception of Be, our recommended values for alkaline-earth polarizabilities are those obtained from the hybrid RCI+MBPT method. We note very good agreement of the RCCSDT calculations of Ref. [35] for the ground state polarizabilities of $\mathrm{Ca}, \mathrm{Sr}$, and $\mathrm{Ba}$ with the recommended values in all three cases. One of the problems of the hybrid approach is the paucity of high-precision experimental data for divalent atoms. Strontium is the only atom where the polarizability has been quoted with a precision approaching $0.1 \%$ [57]. This is due to the availability of a high precision estimate of the resonant oscillator strength obtained by Yasuda and Katori using photoassociation spectroscopy 301]. However, an alternate photo-association experiment [302] gave a lifetime $0.8 \%$ smaller than the Yasuda and Katori value, so it may be over-optimistic to assign an uncertainty of $0.1 \%$ to the strontium polarizability. Currently the best estimate of the $5 s 5 p^{3} P_{0}^{o}$ excited state polarizability of $\mathrm{Sr}$ is accurate to $0.8 \%$ despite the use of the experimental data. The $\mathrm{Sr}$ polarizabilities are discussed in detail in Ref. [141].

The ${ }^{27} \mathrm{Al}^{+}$ion is included in Table XI since it is being used in the development of a single ion optical frequency standard [303]. The most reliable calculation of the ground state polarizability $\alpha_{0}$ is probably given by the CICP calculation. The only experimental value is of low precision (3\%) and was obtained by summing exper- 
imental oscillator strengths. A CICP calculation of the isoelectronic $\mathrm{Si}^{2+}$ system gave a polarizability that was within $0.2 \%$ of the value from a RESIS experiment.

The scatter amongst the different calculations of ytterbium underlines the difficulties of performing calculations in this system. The source of the problem lies in the weakly bound $4 f^{14}$ core. There are $20 \%$ differences between two of the RCI+MBPT calculations that are discussed in recent work by Dzuba and Derevianko [143] and are attributed to the inconsistent use of experimental matrix element for the principal transition in [13]. $\mathrm{Yb}$ is of particular interest for many applications, including ultracold atoms, optical frequency standards, and parity violation experiments.

There is a significant discrepancy for Cd between the refractive index value of 49.65(1.49) a.u. 64] and the calculated value of 44.63 a.u. from the RCICP calculation [113]. For a number of reasons, including the measured values of the oscillator strengths for the $5 s^{2}{ }^{1} S-5 s 5 p$ ${ }^{1} P^{o}$ transitions, it has been suggested that the experimental polarizability might be overestimated [304].

The polarizabilities of other excited states, tensor polarizabilities, and Stark shifts in divalent systems are discussed in the next subsection.

\section{Other data}

Ground state polarizabilities for the other selected systems from Refs. 36, 50, 76, 84, 124, 168, 241 245, 247251, 251, 252, 252 258, 305 are given in Table XII. In this review, we list data for selected systems with a monovalent $n s$ ground state: $\mathrm{Cu}, \mathrm{Ag}, \mathrm{Au}, \mathrm{Zn}^{+}, \mathrm{Hg}^{+}$, and $\mathrm{Yb}^{+}$, and $\mathrm{Al}^{2+}$; ions for which recent RESIS experiments have been performed: $\mathrm{Si}^{3+}$ and $\mathrm{Kr}^{6+}$; neutral atoms with three and four valence electrons: $\mathrm{Al}, \mathrm{Ga}, \mathrm{In}, \mathrm{Tl}, \mathrm{Si}, \mathrm{Sn}$, $\mathrm{Pd}$, and Ir; and $\mathrm{U}$. The reader is referred to a recent review 21] for atomic ground state polarizabilities of other systems not listed herein.

One notable discrepancy between theory and experiment occurs for the $\mathrm{Al}$ ground state where the best calculations exceed the experiment value from an $\mathrm{EH}$ balance experiment by $25 \%$ 36, 254]. The most precise experimental value in Table XII is the RESIS value for $\mathrm{Si}^{3+}$. The final value, $7.433(25)$ a.u. comes from a reanalysis of the raw experimental data [248, 306] that includes estimates of $r^{-7}$ and $r^{-8}$ polarization corrections from RLCCSDT and CICP calculations 84]. The agreement between the RLCCSD polarizability of 7.419 [84] and the latest RESIS reanalysis is at the $0.2 \%$ level.

Table XIII shows a number of measurements and calculations of the tensor polarizability of non-alkali systems including $\mathrm{Ca}, \mathrm{Sr}, \mathrm{Ba}, \mathrm{Zn}, \mathrm{Cd}, \mathrm{Hg}, \mathrm{Tl}, \mathrm{Yb}, \mathrm{Yb}^{+}$from Refs. 36, 53, 141, 145, 206, 234, 234, 240, 251, 253, 259, 259, 259 266, 268, 270 273, 275 279, 293]. These systems are the ones under consideration as frequency standards or are being used in atomic parity violation experiments. Measurements for some states have been omitted from the Table, and some older or less precise results on $\mathrm{Sc}, \mathrm{Y}, \mathrm{La}$ and $\mathrm{Lu}$ [307], Cd [206], Ba 259, 266, 308], Hg [206], Yb [276], Sm and Eu [273] have also been omitted.

One feature of Table XIII is the relatively small number of modern calculations performed. For example, the best calculated polarizabilities for the $4 s 4 p^{1,3} P_{1}^{o}$ states of $\mathrm{Ca}$ are the non-relativistic CICP calculations. Another feature is the relatively large uncertainties in many of the experimental values. There are only five tensor polarizabilities with uncertainties less than $2 \%$. The most precisely measured $\alpha_{2}$ of $-43.04(40)$ a.u. occurs for the Ba $6 s 6 p{ }^{1} P_{1}^{o}$ state. The RCI+MBPT value of -51 a.u. is incompatible with experiment.

The static polarizability differences (in a.u.) for selected transitions in $\mathrm{Cs}, \mathrm{Mg}, \mathrm{Ca}, \mathrm{Ba}, \mathrm{Yb}, \mathrm{Hg}, \mathrm{Ga}, \mathrm{Tl}$, and $\mathrm{Yb}^{+}$derived from Stark shift measurements 93, 183, 216, 278, 279, 290 293, 295, 296, 298 are compared with theoretical calculations [53, 145, 216, 234, 288, 297] in Table XIV. Total polarizability differences are given for the cases where $m$ values are listed, otherwise scalar polarizability differences are listed.

There have been sub-1\% experiments on four systems, $\mathrm{Cs}, \mathrm{Ba}, \mathrm{Yb}$, and $\mathrm{Hg}$. The ability of RCI+MBPT calculations to reproduce experiment for the divalent systems is mixed. The agreement for the $\mathrm{Hg} 6 s^{2}{ }^{1} S-6 s 6 p{ }^{3} P_{1}^{o}$ is excellent, but $10 \%$ discrepancies exist for the $\mathrm{Ba} 6 s^{2}$ ${ }^{1} S-6 s 6 p{ }^{1} P_{1}^{o}$ and $\mathrm{Yb} 6 s^{2}{ }^{1} S-6 s 6 p{ }^{3} P_{1}^{o}$ transitions. However, the RCI+MBPT calculations for Ba [53] and $\mathrm{Yb}$ [240] were among the first RCI+MBPT calculations reported.

\section{EVALUATING UNCERTAINTIES OF THEORETICAL VALUES}

\section{A. Sources of theoretical uncertainty}

As illustrated by the tables in the previous section, benchmark comparisons of theory and experiment carry more value when the theoretical results are accompanied by uncertainty evaluations. Uncertainty bounds are particularly important for the recommended values obtained by either high-precision theory methods or by combination of theory values with experimental data. The analysis of the theoretical uncertainties has been stimulated by the applications that require an error bound to be placed on the recommended values. Such applications include parity violation, development of the next-generation frequency standards, ultra-cold atom studies, etc. Analysis of certain experiments requires input of some data that cannot be easily measured and have to be obtained from theory. In those cases, the uncertainties of the theoretical data have to be included in the uncertainty of the final experimental value. Evaluations of the theoretical uncertainties are still few and cannot be carried out for all of the methods and in all cases. Here, we discuss how some theoretical uncertainties may be evaluated.

There are two distinct sources of theoretical uncertain- 
ties. First, there is an uncertainty associated with the numerical constraints upon the calculations. Many of the methods that we discussed in this review are computationally very intensive and restrictions are imposed so that the calculations can be performed within a reasonable time. Most common numerical uncertainties are associated with the choice of the basis sets, configuration space, radial grid, termination of the iterative procedures after achieving the specified convergence tolerance, etc. Generally, it is possible to at least estimate uncertainties caused by numerical issues by varying the appropriate parameters and recording the changes in the results. In many cases, it is possible to simply continue to change parameters until the change in the resulting values is sufficiently small or negligible.

For example, it is relatively easy to test the convergence of B-spline basis sets. The dimensionality of the radial basis in a RLCCSD calculation for each partial wave (e.g, $n s, n p_{1 / 2}, n p_{3 / 2}, \ldots$ states) is steadily increased. The final values of a property like the sodium ground state polarizability do not change, within the quoted digits whether the $B$-spline basis has a dimension of 40 , 50 , or 70 orbitals [56]. Using only 20 orbitals, however, will lead to change in the final value that is not negligible. Also, truncating partial wave expansion at $l=3$ will measurably affect the final result, while including all partial waves up to $l=6$ is sufficiently complete in this case. Generally, such tests do not have to be carried out at the level of the most accurate calculation possible and it is sometimes sufficient to study the lowest-order results or low-order MBPT values. In some cases, it may become necessary to completely repeat the entire calculation. However, such numerical problems may be studied by well understood conventional methods. In most cases, numerical errors of the theoretical values can be made small enough not to affect any of the significant figures that are quoted, or can be evaluated and quoted as uncertainty in the last digit.

Investigations using the Hylleraas method typically perform a series of calculations of increasing dimension while keeping the non-linear parameters the same. The convergence of the data against a value of the total polynomial power is studied. The total polynomial power for a correlated wave function such as Eq. (43) would be

$$
\Omega=j_{1}+j_{2}+j_{3}+j_{12}+j_{13}+j_{23} .
$$

Most expectation values in a Hylleraas calculation converge as $\sim 1 / \Omega^{p}$. This result is exploited to give uncertainties in energies, transition matrix elements, polarizabilities, and other quantities [117, 118].

The theoretical uncertainties of the second type are much harder to evaluate. These are the uncertainties associated with the particular theoretical methodology, for example, the uncertainty associated with stopping a perturbation theory treatment at third order. Ideally, the total uncertainty of the theoretical value should give an estimate of how far any value is from the actual (unknown) exact result. Evaluation of the complete theoret- ical uncertainty is non-trivial since it essentially involves the evaluation of a quantity that is not known beforehand and cannot be determined by the theoretical methodology adopted.

\section{B. Sources of uncertainties in the sum-over-states polarizability calculations}

It is particularly problematic to evaluate full theoretical uncertainties for the semi-empirical theoretical methods. In this case, there may be no basis to make assumptions regarding the missing theory. It may be possible to infer some information based on the agreement of CICP calculations with quality experiments for similar states in other members of the same iso-electronic series. For example, the CICP ground state polarizability for $\mathrm{Al}^{+}$of 24.14 a.u. has been assessed at $\pm 0.5 \%$ [235] based on the $0.3 \%$ agreement between a CICP calculation of the $\mathrm{Si}^{2+}$ polarizability and a RESIS experiment [88, 239]. The assessment of uncertainties, for states that lack validating information, as in the case of the ${ }^{3} P_{0}^{o}$ state of $\mathrm{Al}^{+}$has a larger speculative element [235].

Several strategies exist for uncertainty evaluation for the ab initio MBPT, correlation potential, and all-order linearized coupled-cluster (RLCCSDT) approaches. These strategies are illustrated using RLCCSDT method which utilizes the sum over states algorithm. For brevity, we refer to RLCCSDT calculation as "all-order" in the text below.

We use the example discussed in Section II A2, i.e. the polarizability of the $5 p_{1 / 2}$ state. Table III lists a detailed breakdown of the contributions to this value. There are three separate contributions: the main part $(5 s-11 s$ and $4 d_{3 / 2}-9 d_{3 / 2}$ ), remainder (all other valence terms), and core contribution. The uncertainty in each term of the main part has to be determined. The energy levels of low-lying states are generally well known. Therefore, the determination of the uncertainty here reduces to the evaluation of the uncertainty in the corresponding electric-dipole matrix elements. The relative uncertainty in the polarizability contribution is twice the relative uncertainty in the matrix element (see Eq. (17)).

The uncertainty of the remainder (higher $n$ contributions) as well as the uncertainty of the ionic core have to be determined separately. The uncertainty in the RPA value of the core is estimated from comparison of the RPA values for noble gases with experiment and precision coupled-cluster calculations (see Table IV and the corresponding discussion). The evaluation of the uncertainty of the remaining highly-excited contribution has been discussed in great detail in recent work on the $\mathrm{Sr}^{+}$ polarizabilities [193].

In most cases, all of the uncertainties are added in quadrature to obtain the final uncertainty of the polarizability value. 


\section{Determination of the uncertainties in E1 matrix elements}

Ultimately, the theoretical uncertainty estimates in the polarizability need uncertainties in the E1 matrix elements such as those listed in Table [II] The starting point of relativistic MBPT or all-order RLCCSD calculations for monovalent systems is a DHF calculation. We refer to the DHF value as the lowest order. Essentially all corrections to that value come from Coulomb correlations. Breit interaction corrections to the E1 matrix elements are generally insignificant at the present level of accuracy [309], and the relativistic corrections are intrinsically included due to use of a relativistic Hamiltonian. Therefore, an uncertainty evaluation requires an estimation of the missing part of the correlation correction. The strategies to do so include:

- approximate evaluation of the size of the correlation correction;

- evaluation of the size of the higher-order corrections;

- study of the order-by-order convergence of perturbation theory;

- study of the breakdown of the various all-order contributions and identification of the most important terms;

- semi-empirical determination of dominant missing contributions.

The first three strategies are aimed at providing rough estimate of the matrix element uncertainty. Separate third-order RMBPT and all-order calculations have to be carried out to evaluate the accuracy of the all-order values since the extraction of third-order matrix elements from the all-order values is impractical.

The application of the first three strategies are illustrated in Table $\mathrm{XV}$ where $\mathrm{Rb} \mathrm{E} 1$ matrix elements are listed [310]. Three values are given for each matrix element: lowest-order DHF value, the third-order RMBPT value, and all-order values obtained from an RLCCSD calculation. Third-order values include the second-order, third-order, and RPA corrections iterated to all-orders (see 51] for detailed description of the third-order MBPT calculations). The size of the correlation correction is estimated as the relative difference between the lowestorder and the all-order values. It is given as a percentage change in the rows labelled "correlation". The size of the fourth and higher-order corrections is estimated as a percentage difference between the third-order and all-order values and listed in the rows labelled "higher orders".

Study of the "correlation" and "higher orders" rows gives some insight into the accuracy of the final all-order values. First, it is noted that the corrections vary significantly among the different transitions. Very rough estimate of the uncertainty can be obtained by assuming
TABLE XV: Rb electric-dipole matrix elements (in a.u.) calculated in different approximations 310]. The rows labelled "correlation" list an estimate of the correlation contribution, determined as the relative difference between the lowest-order and the all-order values. The rows labelled "higher orders" list an estimate of the 4 th and higher-order contributions, determined as the relative difference between the third-order and the all-order values. Absolute values are listed. The negative sign in front of the lowest-order $6 d_{3 / 2}-6 p_{1 / 2}$ value indicates that the lowest-order gives incorrect sign for this matrix element.

\begin{tabular}{|c|c|c|c|}
\hline & $5 s-5 p_{1 / 2}$ & $5 s-6 p_{1 / 2}$ & $6 s-5 p_{1 / 2}$ \\
\hline Lowest order & 4.819 & 0.383 & 4.256 \\
\hline Third order & 4.181 & 0.363 & 4.189 \\
\hline All order & 4.221 & 0.333 & 4.119 \\
\hline Correlation & $14 \%$ & $15 \%$ & $3.3 \%$ \\
\hline \multirow[t]{2}{*}{ Higher orders } & $0.9 \%$ & $9 \%$ & $1.7 \%$ \\
\hline & $8 s-8 p_{1 / 2}$ & $4 d_{3 / 2}-5 p_{1 / 2}$ & $6 d_{3 / 2}-6 p_{1 / 2}$ \\
\hline Lowest order & 26.817 & 9.046 & -0.047 \\
\hline Third order & 25.587 & 8.092 & 2.184 \\
\hline All order & 25.831 & 7.847 & 2.974 \\
\hline Correlation & $3.8 \%$ & $15 \%$ & $100 \%$ \\
\hline Higher orders & $0.9 \%$ & $3 \%$ & $27 \%$ \\
\hline
\end{tabular}

that higher-order corrections incorporated into RLCCSD are smaller than the higher orders that are omitted by RLCCSD. Thus, the difference between third and allorders is taken as the uncertainty. In most cases, this procedure will significantly overestimate the uncertainty since Table XV shows that contributions from all higher orders are lower than the second and third-order in all cases except the small $5 s-6 p_{1 / 2}$ matrix element. However, this procedure clearly indicates that while $5 s-5 p_{1 / 2}$ matrix element is probably accurate to better than $1 \%$, $\mathrm{SD}$ all-order $6 d_{3 / 2}-6 p_{3 / 2}$ matrix element may only be accurate to about $25 \%$.

The last two strategies should be employed if more accurate uncertainty evaluations are required. This can only be done for certain cases within the framework of the RLCCSDT method and requires substantial additional calculations and the careful analysis of all available data. First, the breakdown of the all-order terms have to be studied. Triple excitations need to be added at least partially. If certain types of the contributions (associated with so-called Brueckner orbital terms) are dominant, they may be estimated by the semi-empirical scaling described, for example, in Ref. 37. This procedure involves rescaling single-excitation coefficients $\rho_{m v}$ (see Section IVF) using experimental energies, and rerunning the entire matrix element calculation with the modified coefficients. Obviously, this method is only expected to produce more accurate values if correlation correction is dominated by the terms containing single valence excitation coefficients. However, this is true in many cases. Non-linear terms may also be evaluated. 
The most extensive uncertainty study of this type has recently been carried out for the atomic quadrupole moments of $\mathrm{Ca}^{+}, \mathrm{Sr}^{+}$, and $\mathrm{Ba}^{+}$in 311].

Detailed studies of the uncertainties of the electricdipole matrix elements are described, for example, in Refs. [30, 193, 216]. A brief description is given here for the case of the $4 d_{5 / 2}-5 p_{3 / 2}$ matrix element in $\mathrm{Sr}^{+}[193]$. This transition is important in the evaluation of the $\mathrm{Sr}^{+}$ BBR shift. Correlation corrections change the matrix element by about $20 \%$. Study of the correction breakdown indicates that the correlation is dominated by a single term that contains single valence excitations. Therefore, we carry out additional $a b$ initio calculations that partially include triple excitations, and also perform scaled RLCCSD and RLCCSDT calculations. The results of these four calculations are listed below. All data are in atomic units. The first line corresponds to the "all-order" lines in Table XV

$\begin{array}{lr}\text { RLCCSD } & 4.150 \\ \text { RLCCSDT } & 4.198 \\ \text { RLCCSD scaled } & 4.187 \\ \text { RLCCSDT scaled } & 4.173 \\ \text { Final } & 4.187(14)\end{array}$

Note that scaled values are much closer together than the SD and SDT ab initio values. The final value was taken to be RLCCSD scaled 4.187(14) result (see, for example, 37] and references therein for the discussion of this choice). The uncertainty of 0.014 is determined as the maximum difference between the scaled SD values and the $a b$ initio SDT and scaled SDT values.

\section{APPLICATIONS}

\section{A. Parity non-conservation}

The goals of the parity nonconservation (PNC) studies in heavy atoms are to search for new physics beyond the standard model of the electroweak interaction by precise evaluation of the weak charge $Q_{w}$, and to probe parity violation in the nucleus by evaluating the nuclear anapole moment. The study of PNC in the cesium $6 s-7 s$ transition involving both high-precision measurement [287] and several high-precision calculations provided an atomicphysics test of the standard model of the electroweak interactions [312]. Moreover, accurate determination of the uncertainty in theoretical values was necessary, leading to detailed studies of parity-conserving quantities in Cs including the polarizabilities of the $6 s, 6 p_{J}$, and $7 s$ states (see [31, 37, 313, 314] and references therein). The analysis of the Cs experiment was instrumental in developing methods to evaluate the uncertainties of the theoretical data 315].

In the Cs experiment 287], the PNC amplitude was measured relative to Stark-induced tensor transition po- larizability $\beta_{S}$ (some works refer to this quantity as the vector transition polarizability). The DC electric field mixes states of opposite parity allowing electric-dipole transitions between $n s$ states. The Stark-induced amplitude is expressed via the Stark-induced scalar and tensor transition polarizabilities $\alpha_{S}$ and $\beta_{S}$. In the case of the Cs $7 s-6 s$ transition, they are calculated as sum-overstates using the expressions [315]

$$
\begin{aligned}
\alpha_{S} & =\frac{1}{6} \sum_{n}\left\langle 7 s\|D\| n p_{1 / 2}\right\rangle\left\langle n p_{1 / 2}\|D\| 6 s\right\rangle \\
& \times\left(\frac{1}{E_{7 s}-E_{n p_{1 / 2}}}+\frac{1}{E_{6 s}-E_{n p_{1 / 2}}}\right) \\
& -\frac{1}{6} \sum_{n}\left\langle 7 s\|D\| n p_{3 / 2}\right\rangle\left\langle n p_{3 / 2}\|D\| 6 s\right\rangle \\
& \times\left(\frac{1}{E_{7 s}-E_{n p_{3 / 2}}}+\frac{1}{E_{6 s}-E_{n p_{3 / 2}}}\right), \\
\beta_{S} & =\frac{1}{6} \sum_{n}\left\langle 7 s\|D\| n p_{1 / 2}\right\rangle\left\langle n p_{1 / 2}\|D\| 6 s\right\rangle \\
& \times\left(\frac{1}{E_{7 s}-E_{n p_{1 / 2}}}-\frac{1}{E_{6 s}-E_{n p_{1 / 2}}}\right) \\
& +\frac{1}{12} \sum_{n}\left\langle 7 s\|D\| n p_{3 / 2}\right\rangle\left\langle n p_{3 / 2}\|D\| 6 s\right\rangle \\
& \times\left(\frac{1}{E_{7 s}-E_{n p_{3 / 2}}}-\frac{1}{E_{6 s}-E_{n p_{3 / 2}}}\right) .
\end{aligned}
$$

These quantities have been extensively studied due to their importance in PNC research, [287, 313 316]. It is more complicated to calculate $\beta_{S}$ accurately, in comparison to $\alpha_{S}$, owing to severe cancellations between different terms contributing to $\beta_{S}$. The ratio of $\alpha_{S}$ and $\beta_{S}$ has been measured to high precision [316]. At the present time, Cs experiment is consistent with the standard model [312].

However, the precise measurement of PNC amplitudes in Cs [287] also led to an experimental value of the small contribution from the nuclear-spin dependent PNC accurate to $14 \%$. The constraints on weak nucleon-nucleon coupling constants derived from this experiment and calculations in Cs were found to be significantly inconsistent with constraints from deep inelastic scattering and other nuclear experiments 317 319]. At the present time, this discrepancy remains unexplained.

More PNC experiments in other atomic systems, such as $\mathrm{Ra}^{+}, \mathrm{Yb}$, and $\mathrm{Fr}$ are currently in progress. Experiments in $\mathrm{Pb}, \mathrm{Bi}$, and $\mathrm{Tl}$ have been conducted but theoretical calculations of comparable accuracy are not available to permit precise comparison of experiments with the standard model. Comparison of theoretical and experimental values of $\alpha_{S}$ and $\beta_{S}$ for $\mathrm{Tl}$ are given in Ref. [297]. 


\section{B. Ultracold atoms in optical lattices and quantum computation}

Quantum computation is a field of research which is aimed at using the quantum nature of matter to produce fundamentally new methods of computation. There are various approaches to the experimental realization of the quantum computation. In the quantum computation scheme relevant to this review, the qubits are realized as internal states of neutral atoms trapped in optical lattices or microtraps. This approach to quantum computation has many advantages, such as the long decoherence times of the internal states of the atoms, flexibility in controlling atomic interactions, scalability, possible massive parallelism, and well-developed experimental techniques.

Trapping an atom or a group of atoms in an optical lattice raises the possibility that the laser field used to create the lattice might shift the energy levels of the lower and upper states by different amounts. This can result in a wavelength (and intensity) dependent shift of the clock transition. This issue was first raised for the atomic clocks based on neutral atoms trapped in optical lattices.

A solution to this problem was proposed by Katori et al. [55] who suggested that the laser can be tuned to a magic wavelength $\lambda_{\text {magic }}$, where lattice potentials of equal depth are produced for the two electronic states of the clock transition. At such wavelength, the AC polarizabilities of the two relevant states satisfy the condition

$$
\alpha_{\text {upper }}(\lambda)=\alpha_{\text {lower }}(\lambda) .
$$

Ab initio calculations of the dynamic polarizability are valuable in making an initial estimate of the magic wavelength prior to construction of the optical lattice. However, it is possible to make very precise determinations of the magic wavelength once the lattice has been constructed and atoms have been trapped since the experimental design is that of a null experiment. The experimental magic wavelength can be used as a constraint upon the dynamic polarizability and used to refine the polarizability calculation.

Examples of a magic wavelength calculation are depicted in Fig. 3, where polarizabilities of the Li $2 s$ and $3 p_{1 / 2}$ states obtained using RLCCSDT method are plotted. The magic wavelengths are located at the crossing points of the two curves. The ground state polarizability is nearly flat in this wavelength region, while the $3 p_{1 / 2}$ polarizability has several resonances noted by the vertical lines.

One of the current goals of the quantum information projects is to design an apparatus capable of interconnecting "flying" and "stationary" qubits. The ability to trap neutral atoms inside high- $Q$ cavities in the strong coupling regime is of particular importance for such schemes. In a far-detuned optical dipole trap, the potential experienced by an atom can be either attractive or repulsive depending on the sign of the $\mathrm{AC}$ Stark shift due to the trap light. The excited states

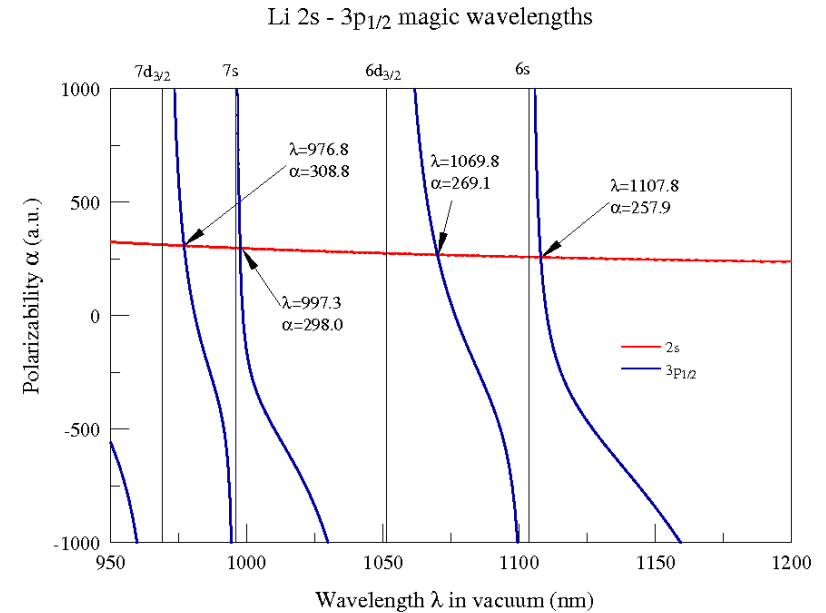

FIG. 3: Magic wavelengths for the $2 s-3 p_{1 / 2}$ transition in Li. Upper state of the resonant transition is marked on top of the box.

may experience an AC Stark shift with an opposite sign to the ground state Stark shift which will affect the fidelity of the experiments. McKeever et al. [320] demonstrated state-insensitive trapping of Cs atoms at $\lambda_{\text {magic }}=935 \mathrm{~nm}$ while still maintaining strong coupling for the $6 p_{3 / 2}-6 s_{1 / 2}$ transition.

The magic wavelengths in $\mathrm{Na}, \mathrm{K}, \mathrm{Rb}$, and $\mathrm{Cs}$ atoms for which the $n s$ ground state and either of the first two $n p_{j}$ excited states experience the same optical potential for state-insensitive cooling and trapping were evaluated in 29]. This was accomplished by matching the dynamic polarizabilities of the atomic $n s$ and $n p_{j}$ states using extensive relativistic all-order calculations. Uncertainties in the dynamic polarizabilities were also evaluated.

One requirement for the experimental realization of the scalable quantum computer is the design of a quantum gate with low error rate which will allow for error correction. Therefore, it is important to study the various decoherence mechanisms and to search for ways to optimize gate performance.

The issue of the mismatch of the polarizabilities of the ground and excited states has also arisen in schemes to perform quantum logical operations where it is a source of decoherence. In the Rydberg gate scheme 321, the qubit is based on two ground hyperfine states of neutral atoms confined in an optical lattice. A two-qubit phase gate may be realized by conditionally exciting two atoms to relatively low-lying Rydberg states. The choice of this particular scheme results from its potential for fast (sub-microsecond) gate operations. Such a gate has been recently experimentally demonstrated [322]. An atom in a Rydberg state will, in general, move in a different optical lattice potential than that experienced by the ground state. Therefore, the vibrational state of the atom in the lattice may change after the gate operation is completed, leading to decoherence due to motional heating. The optical potential for a given state depends on its AC 
polarizability, so we can seek to minimize this motional heating effect by the choice of a particular Rydberg state or of the lattice photon frequency $\omega$. A method for accomplishing this by matching the frequency-dependent polarizabilities $\alpha(\lambda)$ of the atomic ground state and Rydberg state is described in [323, 324].

In recent work [325], a novel approach to quantum information processing, in which multiple qubits can be encoded and manipulated using electronic and nuclear degrees of freedom associated with individual alkalineearth-metal atoms trapped in an optical lattice, was proposed and analyzed. In this scheme, curves of dynamic polarizabilities are needed for alkali and group II atom elements to locate the wavelengths where one of the species can escape or where AC Stark shifts cancel for a specific transition.

\section{Atomic clocks}

The current definition of the second in the International System of Units (SI) is based on the microwave transition between the two hyperfine levels of the ground state of ${ }^{133} \mathrm{Cs}[343$ ]. The present relative standard uncertainty of the Cs microwave frequency standard is around $5 \times 10^{-16}$. More accurate clocks are needed for a variety of applications. Significant recent progress in optical spectroscopy and measurement techniques has led to the achievement of relative standard uncertainties in optical frequency standards that are comparable to the Cs microwave benchmark. The frequencies of feasible optical clock transitions are five orders of magnitude greater than the standard microwave transitions, and so smaller relative uncertainties are potentially achievable. A list of optical transitions recommended for this purpose has recently been disseminated by the International Committee for Weights and Measures 344.

There are two types of optical atomic clocks under active investigation at the moment. Both types of clocks are based on optical frequency transitions with a narrow linewidth. The narrow linewidth mandates that the upper state of the clock transition be a long-lived metastable state. One type of clock is implemented using a group of cold atoms trapped in an optical lattice. The second consists of a single laser cooled ion. With extremely low systematic perturbations and better stability and accuracy, such optical frequency standards should exceed the performance of the existing Cs standard. A commonly quoted target for the new generation of optical frequency standards is a fractional uncertainty of $\Delta \nu / \nu_{0}=10^{-18}$ [5, 334, 345, 346].

There are two main interconnecting areas of theoretic atomic clock research: prediction of atomic properties required for new clock proposals and determination of quantities contributing to the uncertainty budget. New clock proposals require estimates of the atomic properties for details of the proposals (transition rates, lifetimes, branching ratios, magic wavelengths, scattering rates, etc.) and evaluation of the systematic shifts (Zeeman shift, electric quadruple shift, blackbody radiation shift, AC Stark shifts due to various laser fields, etc.). While a large fraction of these quantities may be eventually measured, lack of knowledge of some of these properties may delay experimental realization of new proposals. In the case of well-developed proposals, one of the main uncertainty issues is the blackbody radiation (BBR) shift. The operation of atomic clocks is generally carried out at room temperature, whereas the definition of the second refers to the clock transition in an atom at absolute zero. This implies that the clock transition frequency should be corrected for effects of finite temperature, of which the leading contributor is the blackbody radiation shift. The BBR shift is looming as a major component in the uncertainty budget of the optical frequency standards. Table XVII shows the fractional uncertainty budget for a ${ }^{87} \mathrm{Sr}$ optical frequency standard [347]. The BBR shift is by far the largest source of uncertainty in the uncertainty budget. It is noteworthy that the second largest source of uncertainty is the AC stark shift caused by the optical lattice (this estimate did not take into account possible corrections due to M1 and E2 multipoles caused by spatial inhomogeneities of the lattice field). Experimental measurements of BBR shifts are difficult and highprecision theoretical calculations are presently needed.

\section{Black body radiation shifts}

The BBR shift is the AC Stark shift resulting from the ambient blackbody radiation field surrounding the atom. The BBR energy shift of an atomic state can be approximately calculated as [57]

$$
\Delta E=-\frac{2}{15}(\alpha \pi)^{3} \alpha_{0}(0) T^{4}(1+\eta)
$$

where $\alpha$ is the fine structure constant. The static scalar polarizability $\alpha_{0}(0)$ and energy shift $\Delta E$ in Eq. (66) are in atomic units. In this expression, the temperature in $\mathrm{K}$ is multiplied by $3.1668153 \times 10^{-6}$. This is converted to $\mathrm{Hz}$ by multiplying by $6.579684 \times 10^{15}$. The factor $\eta$ is a correction factor that allows for the frequency dependence of the polarizability when the blackbody integral is performed [11, 13, 235]. The factor $\eta$, referred to as the dynamic shift, is most conveniently written as [13, 235]

$$
\eta \approx-\frac{40 \pi^{2} T^{2}}{21 \alpha_{d}(0)} S(-4)
$$

The dynamic shift is largest when the excitation energies of the states that make the largest contribution to the polarizabilities are small. The dynamic shift is largest for strontium and increases the BBR shift by $2.7 \%$ [13].

Under most circumstances, the energy shift of an atomic level by a radiation field is dominated by the dipole component. However, other multipoles might make a contribution when the atomic level is part of 
TABLE XVI: The blackbody radiation shifts for a number of proposed optical frequency standards. The polarizability difference, $\delta \alpha$, is negative when the upper state polarizability is smaller than the lower state polarizability. A negative polarizability difference means the frequency shift is positive. All BBR shifts are evaluated at $300 \mathrm{~K}$ and values that include the dynamic shifts are indicated with an asterisk $\left({ }^{*}\right)$. Linewidths are converted from lifetimes, $\tau$ using $\delta \nu_{\text {nat }}=1 /(2 \pi \tau)$; natural linewidths are given for fermionic isotopes for the $n s^{2}-n s n p$ clock transitions. Uncertainties in the last digits are given in parentheses. References are given in square brackets. The composite CI calculation for $\mathrm{Yb}^{+}$is a hybrid calculation that used CI to explicitly allow for core excitations but also included core polarization using a semi-empirical core polarization potential.

\begin{tabular}{|c|c|c|c|c|c|c|}
\hline Transition & $\nu_{0}\left(\times 10^{15} \mathrm{~Hz}\right)$ & $\Delta \nu_{\text {nat }}(\mathrm{Hz})$ & $\Delta \alpha\left(a_{0}^{3}\right)$ & $\Delta \nu_{\mathrm{BBR}}(\mathrm{Hz})$ & $\frac{\Delta \nu_{\mathrm{BBR}}}{\nu_{0}} \mid \times 10^{15}$ & Approach \\
\hline $\mathrm{Ca}^{+}\left(4 s_{1 / 2}-3 d_{5 / 2}\right)$ & $0.411[326]$ & $0.14[9]$ & $-44.1(1.5)$ & $0.38(1)$ & 0.925 & RLCCSDT [192] \\
\hline $\operatorname{Sr}^{+}\left(5 s_{1 / 2}-4 d_{5 / 2}\right)$ & $0.445[327]$ & $0.4[9]$ & $-29.3(1.1)$ & $0.250(9)^{*}$ & $0.562^{*}$ & RLCCSDT [193] \\
\hline $\mathrm{Hg}^{+}\left(5 d^{10} 6 s-5 d_{5 / 2}^{9} 6 s^{2}\right)$ & $1.06[328]$ & $1.8[9]$ & & & & Cryogenic \\
\hline $\mathrm{Yb}^{+}\left(4 f^{14} 6 s-4 f^{13} 6 s^{2}{ }^{2} F_{7 / 2}\right)$ & $0.642[329]$ & $\sim 10^{-9}[9]$ & 11.7 & -0.101 & 0.16 & $f$-sum composite CI $[329-331$ \\
\hline $\mathrm{Yb}^{+}\left(4 f^{14} 6 s-4 f^{14} 5 d^{2} D_{3 / 2}\right)$ & $0.688[332]$ & $3.1[9]$ & $42(8)$ & $-0.36(7)$ & $0.53(10)$ & Expt. $[332]$ \\
\hline \multirow[t]{2}{*}{$\mathrm{Al}^{+}\left(3 s^{2}{ }^{1} S-3 s 3 p^{3} P_{0}^{o}\right)$} & 1.12 [333] & $0.008[9]$ & 0.483 & $-0.0042(32)$ & $0.004(3)$ & CICP [235] \\
\hline & & & & $-0.008(3)$ & $0.007(3)$ & Expt. [97] \\
\hline $\operatorname{In}^{+}\left(5 s^{2}{ }^{1} S-5 s 5 p^{3} P_{0}^{o}\right)$ & $1.27[334,335]$ & $0.8[9]$ & $<30.7$ & $>-0.264$ & $<0.20$ & Theory, using $\Delta \alpha(\mathrm{Cd})$ \\
\hline $\operatorname{Mg}\left(3 s^{2}{ }^{1} S-3 s 3 p^{3} P_{0}^{o}\right)$ & $0.655[336]$ & $0.00014[108]$ & $29.9(7)$ & $-0.258(7)^{*}$ & $0.394(11)$ & RCI+MBPT [13] \\
\hline $\mathrm{Ca}\left(4 s^{2}{ }^{1} S-4 s 4 p^{3} P_{0}^{o}\right)$ & & & 135.9 & - & & CICP [234] \\
\hline $\operatorname{Sr}\left(5 s^{2}{ }^{1} S-5 s 5 p{ }^{3} P_{0}^{o}\right)$ & 0.429 [338] & $0.0014[108]$ & $261.1(3.6)$ & $-2.354(32)^{*}$ & $5.49(7)$ & $\mathrm{RCI}+\mathrm{MBPT}[13]$ \\
\hline \multirow[t]{2}{*}{$\mathrm{Yb}\left(6 s^{2}{ }^{1} S-6 s 6 p^{3} P_{0}^{o}\right)$} & $0.518[339]$ & $0.008[340]$ & $155(15)$ & $-1.34(13)^{*}$ & $2.6(3)$ & RCI-MBPT [13] \\
\hline & & & $161(15)$ & $-1.39(13)$ & $2.7(3)$ & Hybrid RCI+MBPT [143] \\
\hline $\operatorname{Zn}\left(4 s^{2}{ }^{1} S-4 s 4 p^{3} P_{0}^{o}\right)$ & $0.969[336]$ & $0.0025[341]$ & 29.57 & -0.255 & 0.263 & RCICP $[113]$ \\
\hline $\mathrm{Cd}\left(5 s^{2}{ }^{1} S-5 s 5 p^{3} P_{0}^{o}\right)$ & $0.903[33]$ & $\sim 10^{-2}$ & 30.66 & -0.264 & 0.292 & $\mathrm{RCICP}$ [113] \\
\hline \multirow[t]{2}{*}{$\operatorname{Hg}\left(6 s^{2}{ }^{1} S-6 s 6 p^{3} P_{0}^{o}\right)$} & $1.13[145]$ & $0.11[342]$ & 21.0 & -0.181 & 0.160 & RCI+MBPT [145] \\
\hline & & & 24.00 & -0.207 & 0.183 & RCICP [113] \\
\hline
\end{tabular}

a spin-orbit multiplet [13]. The $n s n p{ }^{3} \mathrm{P}_{J}$ levels of the alkaline-earth atoms have relatively small energy splittings. The frequency shift due to magnetic dipole (M1) transitions could become important at the $10^{-18}$ level of accuracy. The M1 frequency shift for Sr has been estimated at $2.4 \times 10^{-5} \mathrm{~Hz}$ [13]. The frequency shifts for other alkaline earths can be estimated using the approximate result $\delta \nu_{\mathrm{X}} \approx \delta \nu_{\mathrm{Sr}} \delta E_{\mathrm{Sr}}\left({ }^{3} P_{1}^{o}-{ }^{3} P_{0}^{o}\right) / \delta E_{\mathrm{X}}\left({ }^{3} P_{1}^{o}-{ }^{3} P_{0}^{o}\right)$ since the magnetic dipole matrix elements between the two members of the triplet show little variation between different species.

Table XVI lists the frequencies, linewidths, and blackbody radiation shifts for a number of potential optical frequency standards from Refs. 9, 13, 97, 108, 113, 143, 145, 190, 192, 193, 232, 234, 235, 326 329, 331 333, 336342, 348]. The polarizability difference, $\Delta \alpha$, is negative when the upper state polarizability is smaller than the lower state polarizability. All BBR shifts are evaluated at $300 \mathrm{~K}$. Linewidths are converted from lifetimes, $\tau$ using $\delta \nu_{\text {nat }}=1 /(2 \pi \tau)$; natural linewidths are given for fermionic isotopes for the $n s^{2}-n s n p$ clock transitions. It is immediately apparent that the proposed ion clocks generally have smaller polarizability differences than the electrically neutral atoms in the lattice clocks.

All of the proposed frequency standards, with the exception of the $\mathrm{Al}^{+}\left(3 s^{2}-3 s 3 p^{3} P_{0}^{o}\right)$ transition have $T=300$ $\mathrm{K}$ fractional shifts of $10^{-16}$ or higher. The frequency shifts of the neutrals are generally larger than the singly charged ions.

\section{Optical lattice clocks}

Many of the issues that impact on the optimal choice for an optical frequency standard are present in the proposed strontium ${ }^{1} S_{-}{ }^{3} P_{0}^{o}$ optical frequency standard 338, 349]. While Sr might be a desirable atom from the perspective of practical experimentation, it is the most susceptible to BBR shifts since the polarizability difference between the two states is 259.8 a.u., giving a BBR shift of $2.35 \mathrm{~Hz}$ at $T=300 \mathrm{~K}[141]$.

Assuming that the polarizability difference can be determined to $0.2 \%$ accuracy, the resulting BBR uncertainty would be $0.0047 \mathrm{~Hz}$ which corresponds to a frac- 
TABLE XVII: Fractional uncertainty budget for the ${ }^{87} \mathrm{Sr}$ atomic frequency standard 347]. The BBR shifts are evaluated at $296 \mathrm{~K}$. Corrections that include knowledge of polarizabilities are preceded by an asterisk $(*)$.

\begin{tabular}{lcc}
\hline \hline Effect & $\begin{array}{c}\text { Correction } \\
\left(\times 10^{16}\right)\end{array}$ & $\begin{array}{c}\text { Uncertainty } \\
\left(\times 10^{16}\right)\end{array}$ \\
\hline *Lattice Stark shifts & -6.5 & 0.5 \\
*Lattice hyperpolarizability & & \\
Stark shifts & -0.2 & 0.2 \\
*BBR shifts & 52.1 & 1.0 \\
*Probe laser Stark shifts & 0.2 & 0.1 \\
1st order Zeeman & 0.2 & 0.2 \\
2nd order Zeeman & 0.2 & 0.02 \\
Collisional shift & 8.9 & 0.8 \\
Line pulling & 0.0 & 0.2 \\
Servo error shift & 8.9 & 0.5 \\
2nd order Doppler shift & 8.9 & $<0.01$ \\
Totals & 54.9 & 1.5 \\
\hline \hline
\end{tabular}

tional uncertainty of $1 \times 10^{-17}$. Achieving such a level of precision requires experimental determination of the five most important transitions in the oscillator strength sum-over-states to a precision of $0.1 \%$ [141].

Another problem associated with large polarizability differences is the enhanced sensitivity with respect to variations in temperature. A $1.0 \mathrm{~K}$ uncertainty in the temperature at $300 \mathrm{~K}$ would lead to a frequency uncertainty of $\Delta \nu=0.064 \mathrm{~Hz}$. The large BBR shift makes a $\mathrm{Sr}$ standard particularly sensitive to an imprecisely known temperature. These problems can be reduced by running the clock at lower temperatures. For example, the BBR uncertainties stated above can be reduced in size by a factor of more than 200 by maintaining the clock at liquid nitrogen temperatures.

Sensitivity to BBR fields has resulted in a proposal that mercury would be a superior candidate for an optical frequency standard [145] despite the inconvenience of much shorter optical lattice wavelengths. Cadmium and zinc have also been identified as candidates with reduced BBR shifts [113, 341]. The drawback of the group IIB atoms are the greater uncertainties in the determination of the polarizabilities. The underlying $(n d)^{10}$ shell of the group IIB atoms implies large core polarizabilities, stronger valence-core correlations and valence expectation values that are slower to converge. In addition, the resonant oscillator strength for these atoms is about 1.4, as opposed to 1.7-1.8 for the group II atoms. Consequently the use of a high precision resonant transition matrix element from a photo-association experiment would do less to minimize the uncertainty than in a group II atom.

Ytterbium has also been the subject of increased experimental interest [339]. This system also suffers from the drawback that it has a large polarizability. Further- more, a first-principles calculation of the polarizability to a guaranteed accuracy of even $1 \%$ is a very difficult proposition. The most weakly bound core shell is the $(4 f)^{14}$ shell and the $\mathrm{Yb}^{2+}$ polarizability is $\sim 9$ a.u. 124 .

The atoms that have so far been used in most experiments, are those that are amenable to cooling and trapping. The lighter group II atoms, Be and $\mathrm{Mg}$, have the disadvantage that they are difficult to cool, but have the advantage of much smaller BBR shifts [337]. Further, it would be easier to compensate for the effect of the BBR shift in Be and $\mathrm{Mg}$ than in most other atoms. Besides having smaller shifts, these are relatively light atoms with small core polarizabilities, so the uncertainties associated with any calculation will be smaller than those of other lattice clock. These considerations apply most strongly to beryllium. In this case, the polarizability difference of the clock atom states is only 1.8 a.u. (Table XI). Beryllium has only four electrons, so calculations with ECGs are possible and should ultimately be able to achieve a precision approaching 0.01 a.u.

The dynamic correction to the BBR shift makes a finite contribution when the precision reaches the $10^{-18}$ $\mathrm{Hz}$ level [57, 193] but should not lead to a significant increase in the BBR shift uncertainty. The sum rule for evaluation of $S(-4)$ is more strongly dominated by a few major transitions than $\alpha_{0}$ and the relative uncertainty in $S(-4)$ will not be any larger than that of $\alpha_{0}$. Further, the dynamic contribution will be small so the need for a precise evaluation is reduced.

One recent complication has been the recent realization that higher order multipoles could have an impact upon the magic wavelength. The inhomogenous spatial distributions of the electric and magnetic fields in the standing wave patterns that define the lattice can lead to energy shifts in the atomic vibrational motion [350]. This requires the definition of a motion insensitive magic wavelength which requires knowledge of the frequency dependent electric quadrupole and magnetic dipole polarizabilities 351].

\section{Ion clocks}

Ion state polarizabilities are generally smaller than those for neutral atoms because the electrons are more tightly bound. None of the ion clocks have polarizability differences that exceed 50 a.u..

The $\mathrm{Al}^{+}\left(3 s^{2}-3 s 3 p^{3} P_{0}^{o}\right)$ transition has the smallest BBR shift of any ion clock due to the fortuitous near equality of polarizabilities of the two states in the clock transition. The CICP BBR shift is only $-0.0042(32) \mathrm{Hz}$ [235] while experiment gave $-0.008(3) \mathrm{Hz}$ [97]. However, the technical requirements for construction of an $\mathrm{Al}^{+}$clock are much more demanding since the clock transition and cooling laser are in the ultraviolet [333].

While the $\mathrm{Ca}^{+}$system is monovalent, calculation of its polarizabilities using RLCCSDT method is a more diffi- 
TABLE XVIII: Summary of the recent theoretical calculations of the Stark shift coefficient $k$ in $10^{-10} \mathrm{~Hz} /(\mathrm{V} / \mathrm{m})^{2}$ and the BBR radiation shift parameter $\beta$ for transitions between the ground hyperfine states and comparison with experiment. All BBR shifts are evaluated at $300 \mathrm{~K}$. Uncertainties in the last digits are given in parentheses. References are given in square brackets.

\begin{tabular}{|c|c|c|c|c|}
\hline Atom & Transition & Method & $k$ & $\beta$ \\
\hline \multirow[t]{2}{*}{${ }^{7} \mathrm{Li}$} & $2 s(F=2 \leftrightarrow F=1)$ & RLCCSDT [175] & -0.05824 & $-0.5017 \times 10^{-14}$ \\
\hline & & Expt. [352] & $-0.061(2)$ & \\
\hline \multirow[t]{2}{*}{${ }^{23} \mathrm{Na}$} & $3 s(F=2 \leftrightarrow F=1)$ & RLCCSDT [353] & -0.1285 & $-0.5019 \times 10^{-14}$ \\
\hline & & Expt. [352] & $-0.124(3)$ & \\
\hline \multirow[t]{2}{*}{${ }^{39} \mathrm{~K}$} & $4 s(F=2 \leftrightarrow F=1)$ RLCCSDT [176] & -0.0746 & $-1.118 \times 10^{-14}$ & \\
\hline & & Expt. [352] & $-0.071(2)$ & \\
\hline \multirow[t]{3}{*}{${ }^{87} \mathrm{Rb}$} & $5 s(F=2 \leftrightarrow F=1)$ & RLCCSDT [354] & $-1.272^{*}$ & $-1.287 \times 10^{-14}$ \\
\hline & & RCI+MBPT [130] & $-1.24(1)$ & $-1.26(1) \times 10^{-14}$ \\
\hline & & Expt. [352] & $-1.23(3)$ & \\
\hline \multirow[t]{4}{*}{${ }^{133} \mathrm{Cs}$} & $6 s(F=4 \leftrightarrow F=3)$ & RLCCSDT [355] & $-2.271(8)$ & $-1.710(6) \times 10^{-14}$ \\
\hline & & Theory, PTSCI [129] & $-2.26(2)$ & $-1.70(2) \times 10^{-14}$ \\
\hline & & Expt. [356] & $-2.271(4)$ & $-1.710(3) \times 10^{-14}$ \\
\hline & & Expt. [357] & $-2.05(4)$ & $-1.54(4) \times 10^{-14}$ \\
\hline${ }^{137} \mathrm{Ba}^{+}$ & $6 s(F=2 \leftrightarrow F=1)$ & PTSCI [130] & $-0.284(3)$ & $-0.245(2) \times 10^{-14}$ \\
\hline \multirow[t]{2}{*}{${ }^{171} \mathrm{Yb}^{+}$} & $6 s(F=1 \leftrightarrow F=0)$ & RMBPT3 [124] & -0.1796 & $-0.0983 \times 10^{-14}$ \\
\hline & & Theory, PTSCI [130] & $-0.171(9)$ & $-0.094(5) \times 10^{-14}$ \\
\hline${ }^{199} \mathrm{Hg}^{+}$ & $6 s(F=1 \leftrightarrow F=0)$ & PTSCI [130] & $-0.060(3)$ & $-0.0102(5) \times 10^{-14}$ \\
\hline
\end{tabular}

cult proposition than for the iso-electronic neutral potassium [192]. The difficulties lie in the determination of the $3 d$ state polarizability. First, the $3 d$ state is quite compact and its charge distribution does perturb the charge distribution of the $3 s^{2} 3 p^{6}$ core. This leads to a more slowly convergent perturbation theory or CI expansion. Second, about $30 \%$ of the polarizability comes from the $3 d \rightarrow n f$ excitations. The sum-over-states in this case is not dominated by a single transition, so discrete excitations up to the $12 f$ have to be included. Furthermore, the continuum contribution is significant. Including states up to $12 f$ means a much larger $B$-spline basis needs to be used, which in turn makes the calculation more exacting. Similar considerations impact the BBR shift calculation for $\mathrm{Sr}^{+}$[193].

The relative uncertainties associated with the determination of the BBR shifts for the two proposed $\mathrm{Yb}^{+}$ standards are also large due to the underlying $4 f^{14}$ core. This is partly mitigated by the small size of the BBR shifts. The BBR shift for $\mathrm{In}^{+}$was determined by assuming the polarizability difference would be smaller than that of cadmium. This estimate will be an overestimate since the $\mathrm{In}^{+}$ion will have smaller polarizabilities than cadmium.

\section{Experimental possibilities}

So far discussions have focused largely on theory-based approaches to the determination of the relevant polariz- abilities. However, experimental avenues do exist. For example, the polarizability of the $\mathrm{Si}^{2+}$ ground state, an ion iso-electronic with $\mathrm{Al}^{+}$has been determined by RESIS to an accuracy of better than $0.1 \%$. A RESIS experiment on $\mathrm{Al}^{+}$should be able to achieve a similar precision. Similarly, a RESIS experiment should be able to determine the $\mathrm{In}^{+}$ground state polarizability to an accuracy of $0.1 \%$. However, an improved theoretical analysis would be needed to get RESIS polarizabilities for $\mathrm{Ca}^{+}$, $\mathrm{Sr}^{+}$and $\mathrm{Yb}^{+}$. Application of RESIS to excited parent ions also remains a challenge.

The actual knowledge of the ground and excited state polarizabilities is mainly important because it enables the determination of the BBR Stark shift. Direct Stark shift experiments, on the other hand, might ultimately give the most accurate polarizability differences. Table VII shows that experiments on the $n s-n p_{1 / 2}$ transitions of the alkali atoms have yielded polarizability differences with uncertainties less than 0.1 a.u..

Photo-association (PA) experiment lifetimes have been utilized in estimating polarizabilities with sub-1\% precision. However, PA spectroscopy has never been applied to measure transitions to an excited state, and excited state polarizabilities have significant contributions from more than one transition. 


\section{BBR shifts in microwave frequency standards}

A BBR shift also exists for the different hyperfine states involved in microwave frequency standards. In the case of the optical transitions, the lowest (second) order polarizabilities of the clock states are different. In the case of the ground-state hyperfine microwave frequency standards, the lowest (second) order polarizabilities of the clock states are identical and the lowest-order BBR shift vanishes. To evaluate the BBR shift, third-order $F$-dependent polarizabilities must be calculated.

The third-order $F$-dependent ( $F$ is the angular momentum of the hyperfine state) static polarizability, $\alpha_{F}$ can be written [355]

$$
\alpha_{F}=A g_{I} \mu_{n}(2 T+C+R)
$$

where $A$ is an angular coefficient, $G_{I}$ is the nuclear gyromagnetic ratio, and $\mu_{n}$ is the nuclear magneton. The quantities $T, C$ and $R$ arise from third-order perturbation theory and typically involve two electric-dipole matrix elements $\langle i\|D\| j\rangle$ and a one matrix element involving the magnetic hyperfine operator $\mathcal{T}^{(1)}$. For example, term $T$ is given by 355

$$
T=\sum_{m \neq v} \sum_{n \neq v} A_{1} \delta_{j_{n} j_{v}} \frac{\langle v\|D\| m\rangle\langle m\|D\| n\rangle\left\langle n\left\|\mathcal{T}^{(1)}\right\| v\right\rangle}{\left(E_{m}-E_{v}\right)\left(E_{n}-E_{v}\right)}
$$

Here, $A_{1}$ is the angular coefficient and sums over $m, n$ run over all possible states allowed by the selection rules.

The BBR shift at room temperature effecting the Cs microwave frequency standard has been calculated to high accuracy $(0.35 \%$ and $1 \%)$ in Refs. [129, 355], respectively, implying a $6 \times 10^{-17}$ fractional uncertainty. These calculations are in agreement with a $0.2 \%$ measurement 356.

A summary of recent theoretical calculations 124, 130, 175, 353 355] of the Stark shift coefficient $k$ in $10^{-10} \mathrm{~Hz} /(\mathrm{V} / \mathrm{m})^{2}$ and the BBR radiation shift parameter $\beta$ for transitions between the ground hyperfine states and comparison with experiment [352, 356] is given in Table XVIII All BBR shifts are evaluated at $300 \mathrm{~K}$. The Stark coefficient $k$ is defined as

$$
\delta \nu=k E^{2}
$$

where $\delta \nu$ is the frequency shift in the static electric field. The Stark coefficient for the transition between states $F$ and $I$ is related to the polarizability as

$$
k=-\frac{1}{2}\left[\alpha_{0}(F)-\alpha_{0}(I)\right] .
$$

The parameter $\beta$ of the relative temperature-dependent BBR shift of the microwave frequency standard is defined as

$$
\frac{\delta \nu}{\nu_{0}}=\beta\left(\frac{T(K)}{T_{0}}\right)^{4}\left(1+\epsilon\left(\frac{T(K)}{T_{0}}\right)^{2}\right)
$$

where $T_{0}$ is generally taken to be room temperature, $300 K, \epsilon$ parameterizes the lowest-order (in T) contribution to the dynamic correction $\eta$ in Eq. (66), and $\nu_{0}$ is clock transition frequency. The parameter $\beta$ is calculated directly from the Stark-shift coefficient $k$ defined by Eqs. (69, 70) as

$$
\beta=\frac{k}{\nu_{0}}(831.9 \mathrm{~V} / \mathrm{m})^{2}
$$

\section{Long-range interatomic potentials}

The long-range dispersion interaction between two spherically symmetric atoms has the form

$$
V_{\text {disp }}=-\frac{C_{6}}{R^{6}}-\frac{C_{8}}{R^{8}}-\frac{C_{10}}{R^{10}} \ldots
$$

where the $C_{n}$ coefficients are called the dispersion coefficients. The calculation of the dispersion interaction is closely related to polarizability calculations. For example, the $C_{6}$ parameter for two atoms, $a$ and $b$, in states $m$ and $n$, can be evaluated using the oscillator strengths as

$$
C_{6}=\frac{3}{2} \sum_{i j} \frac{f_{m i} f_{n j}}{\Delta E_{m i} \Delta E_{n j}\left(\Delta E_{m i}+\Delta E_{n j}\right)}
$$

The equation is reminiscent of Eq. (10) and any calculation using Eq. (74) automatically generates the necessary information to generate the dipole polarizability. The dispersion coefficients can also be directly evaluated from the polarizability at imaginary frequencies as

$$
C_{6}=\frac{3}{2} \int_{0}^{\infty} \alpha_{a, 0}(i \omega) \alpha_{b, 0}(i \omega) d \omega
$$

The polarizability of state $n$ at imaginary frequencies is written

$$
\alpha_{0}(i \omega)=\sum_{i} \frac{f_{n i}}{\left(\Delta E_{n i}^{2}+\omega^{2}\right)} .
$$

Equation (73) and subsequent expressions given by Eqs. (74), or (75) are the best way to evaluate long-range atom-atom interactions. Orthodox quantum chemistry techniques are not well suited to determining the very small energies of the long range potential.

The importance of a good description of the longrange atom-atom interaction increases at very low energies. Determination of the dissociation energy for many molecules often involves an extrapolation from the rovibrational energy levels of the highest vibrational states 363. This has been accomplished in the semi-classical (WKB) LeRoy-Bernstein procedure [363]. Similarly, the determination of the scattering length in cold-atom collisions often requires knowledge of the dispersion parameters 364]. 
TABLE XIX: The lowest order dispersion coefficient, $C_{6}$ for homo-nuclear atom-atom pairs. The Hybrid-RLCCSD replaces the calculated matrix element for the resonance transition with an experimental value.

\begin{tabular}{lccccc}
\hline \hline Method & ${ }^{\infty} \mathrm{Li}$ & $\mathrm{Na}$ & $\mathrm{K}$ & $\mathrm{Rb}$ & $\mathrm{Cs}$ \\
\hline Hylleraas [118] & $1393.42(5)$ & & & & \\
Model Potential [358] & 1388 & 1472 & 3813 & 4426 & 6331 \\
CICP [105] & 1394.6 & 1561 & 3905 & 4635 & 5174 \\
RLCCSD [56] & & 1564 & 3867 & 4628 & 6899 \\
Hybrid-RLCCSD [56, 359] & $1390(2)$ & $1556(4)$ & $3897(15)$ & $4691(23)$ & $6851(74)$ \\
Expt. & & $3921[360]$ & $4698(4)[361]$ & $6877(24)[74]$ \\
Expt. & & & & $6860(25)[362]$ \\
\hline \hline
\end{tabular}

Better information about the specific values of the dispersion coefficients for many atoms has become available primarily because of the importance of such data for the field of cold-atom physics. There have been the near exact non-relativistic calculation by Yan and co-workers on $\mathrm{H}, \mathrm{He}$ and Li using Hylleraas basis sets [117, 118, 365369]. An important series of calculations on the ground and excited states of the alkali atoms were reported by Marinescu and co-workers [358, 370 373]. However, these calculations were performed with a model potential approach that omitted some dynamical features (e.g. transitions from the core) that should be included. Later calculations with semi-empirical Hamiltonians by Mitroy and co-workers [105, 171, 179, 232 234, 374] and RLCCSD/RCI+MBPT calculations by Derevianko and coworkers [31, 56, 57, 180, 375, 376] should be preferred since the underlying atomic structure descriptions are superior. These calculations encompass both the alkali and alkaline-earth atoms. Table XIX shows that CICP and RLCCSDT calculations of $C_{6}$ for homo-nuclear pairs of alkali atoms agree at the $1 \%$ level. This agreement extends to hetero-nuclear pairs of alkali atoms [105] and to alkaline-earth atoms [57].

\section{E. Thermometry and other macroscopic standards}

The present definition of temperature is based on the triple point of water which is set to $273.16 \mathrm{~K}$. An alternative approach would be to fix the Boltzmann constant, $k_{B}$ and then measure the thermometric properties of a substance which depend on the product $k_{B} T$. At present, the best estimate of the Boltzmann constant was determined by the speed of sound in helium gas. Acoustic gas thermometry (AGT) has resulted in a value of $k_{B}$ accurate to $1.8 \mathrm{ppm}$ 377, 378.

The speed of sound is not the only thermometric property that can be used to determine $k_{B}$. Two other properties are the dielectric constant for helium gas and the refractive index for helium gas [63, 378, 379]. The most recent refractive index experiment using a microwave cavity 63] has given the dipole polarizability to an accuracy of $9.3 \mathrm{ppm}$. If the ${ }^{4} \mathrm{He}$ polarizability is taken as a known quantity from theory, then the microwave cav- ity experiment admits other interpretations. Taking the polarizability and diamagnetic susceptibility as known quantities, the refractive index experiment yields a value for the universal gas constant, $R=8.314487(76)$, which is not far removed in precision from the recommended value of $8.314472(15)$ [377]. Boltzmann's constant, the definition of the mol and the universal gas constant are all inter-related through the identity, $R=k_{B} N_{A}$.

\section{F. Atomic transition rate determinations}

The sum-over-states approach described in Section I A 2 is generally used to determine the polarizabilities from calculated or experimental oscillator strengths or E1 matrix elements. It is possible to reverse the process for systems which have a precisely known polarizability that is dominated by a single strong transition. A good example occurs for the cesium atom [380] where the dipole polarizability [74] and line strength ratio [381] have been measured to high accuracy.

The ground state static polarizability $\alpha_{0}$ can be written as

$$
\alpha_{0}=\alpha_{6 p}+\alpha_{v}^{\prime}+\alpha_{\text {core }}
$$

where $\alpha_{6 p}$ is the contribution of the resonance excitations to the polarizability, i.e. from $6 s-6 p_{1 / 2}$ and $6 s-6 p_{3 / 2}$ transitions, and $\alpha_{\mathrm{v}}^{\prime}$ includes contributions from all other excited states . Rearranging and expressing $\alpha_{6 p}$ in terms of the $6 s \rightarrow 6 p_{1 / 2}$ line strength gives

$$
S_{6 s-6 p_{1 / 2}}=\frac{\alpha_{0}-\alpha_{v}^{\prime}-\alpha_{\text {core }}}{\frac{1}{3 \Delta E_{6 s-6 p_{1 / 2}}}+\frac{R}{3 \Delta E_{6 s-6 p_{3 / 2}}}} .
$$

The factor $R$ is the ratio of the line strengths of the spinorbit doublet. Using $\alpha_{0}=401.0(6)$ [74], $R=1.9809(9)$ [381], ionic core polarizability of $15.644(4)$ [112] that needs to be corrected for the presence of the valence electron by the term $\alpha_{c v}=-0.72, \alpha_{v}^{\prime}=1.81$ yields $S_{6 s-6 p_{1 / 2}}=20.308(42)$ and $S_{6 s-6 p_{3 / 2}}=40.227(84)$. The corresponding values for the reduced matrix elements in atomic units are $4.510(4)$ and $6.347(5)$ for the $6 s-6 p_{1 / 2}$ 
and $6 s-6 p_{3 / 2}$ transitions respectively. The uncertainties of these values are dominated by the uncertainty in the experimental value of $\alpha_{0}$.

A similar approach has been used to determine the multiplet strengths for the resonance transitions in $\mathrm{Mg}^{+}$, $\mathrm{Si}^{3+}$ [84 and $\mathrm{Si}^{2+}$ [88] from RESIS experimental data [198, 239].

Stark shifts for the $n s-n p_{1 / 2}$ transition 201] have also been used to derive estimates for the $S\left(n p_{1 / 2}-(n-1) d_{3 / 2}\right)$ line strengths with a precision of about $1 \%$ for potassium and rubidium [30]. This analysis relied on the result that $80-90 \%$ of the $n p_{1 / 2}$ polarizability comes from the excitation to the $(n-1) d_{3 / 2}$ state. These values were also used to determine the magic wavelengths for the $n p-n s$ transitions in these alkali atoms [29]. Such determination of matrix elements permitted benchmark comparisons of theory and experiment [30].

The procedures described above also permit the crosschecking of results from completely different types of experiment. The domination of the $6 p$ Cs scalar polarizabilities by the $5 d-6 p$ dipole matrix elements facilitated an exacting consistency check of the $5 d$ lifetime with $6 p$ polarizability data [169]. In that work, $5 d-6 p$ matrix elements obtained from experimental Stark shift data were compared with the values extracted from the $5 d$ lifetimes. The experimental measurements of the $5 d$ lifetime and $6 \mathrm{p}$ scalar polarizabilities were found to be inconsistent within the uncertainties quoted by the experimental groups [169]. Theoretical RLCCSDT matrix elements [169] were found to be in agreement with the Stark shift experiments but not with the lifetime measurements.

\section{CONCLUSIONS}

The advent of cold-atom physics owes its existence to the ability to manipulate groups of atoms with electromagnetic fields. Consequently, many topics in the area of field-atom interactions have recently been the subject of considerable interest and heightened importance. This applies to a quantity like the dipole polarizability which governs the first-order response of an atom to an applied electric field and the preceding few years have seen many calculations of atomic polarizabilities for a variety of systems.

The aim of the present review has been to provide a reasonably comprehensive treatment of polarizability related issues as they relate to topics of contemporary importance. However, our treatment is not exhaustive. The polarizabilities of many atoms such as the halogens have been omitted. The reader is referred to the broader treatment in [21]. Similarly, the treatment of DC and AC Stark shift data is better described as selective as opposed to exhaustive.

Part of the motivation for this review has been the importance in developing new atomic based standards of time [344], and corresponding need for precise knowledge of the blackbody radiation shifts. The primary requirement for the BBR application is for polarizabilities and Stark shifts to be known with a precision of $0.1 \%$ or better. Much of the existing body of experimental data is an order of magnitude less precise. Direct measurements of clock transition Stark shifts would be helpful in reducing the BBR shift uncertainties.

One area where theory might be useful in this endeavor would be in the development of atom based polarizability standards. Such a standard is already in existence for helium where theoretical and experimental polarizabilities have uncertainties of $0.17 \mathrm{ppm}$ and $9.1 \mathrm{ppm}$ [63], respectively. These results are not relevant to atomic clock research and another atom needs to serve as a standard. Hylleraas calculations on lithium could yet serve to provide a theoretical reference point for Stark shift experiments. At the moment the uncertainties in the best calculation and best experiment are $0.11 \%$ [170] and $0.07 \%$ 91]. A better treatment of relativistic effects should result in uncertainty in the Hylleraas calculation decreasing to the $0.01 \%$ level of precision.

One possible avenue for improvement could be in the development of hybrid theoretical approaches combining the best features of different methods. For example, orbital-based approaches cannot match the extreme accuracies achievable with correlated basis sets. Direct incorporation of the the Dirac Hamiltonian in orbital-based calculations is now relatively routine, but this is not the case for calculations with correlated basis sets. Perhaps, comparisons of correlated basis calculations with nonrelativistic orbital-based calculations and with relativistic orbital-based calculations could be used to estimate relativistic corrections to Hylleraas calculations or correlation corrections to orbital-based calculations.

It is likely that the determination of polarizabilities will become increasingly important in the future. As experiments become capable of greater precision, it will become necessary to make more detailed corrections of the effects of electromagnetic fields that are used for manipulation and investigation of atoms.

\section{ACKNOWLEDGMENTS}

The authors express their appreciation to Dr. U. I. Safronova for useful discussions and comments on the manuscript, and in particularly for her thorough check of most of the tables. This work was partly supported by the National Science Foundation under Physics Frontiers Center grant PHY-0822671 to the University of Maryland. This research has made extensive use of NASA's Astrophysics Data System. The work of MSS was supported in part by US National Science Foundation Grant No. PHY-07-58088. The work of JM was supported in part by the Australian Research Council Discovery Project DP-1092620. This research was performed in part under the sponsorship of the US Department of Commerce, National Institute of Standards and Tech- 
nology.

[1] J. C. Maxwell, Phil. Trans. R. Soc. (London) 155, 459 (1864).

[2] A. R. Edmonds, Angular Momentum in Quantum Mechanics (Princeton University Press, Princeton, NJ, 1996).

[3] E. Schrödinger, Annalen. der Physik 385, 437 (1926).

[4] A. A. Madej and J. E. Bernard, in Frequency Measurement and Control, Edited by Andre N. Luiten, Topics in Applied Physics, vol. 79, pp.153-195, edited by A. N. Luiten (2001), pp. 153-195.

[5] T. Udem, R. Holzwarth, and T. W. Hansch, Nature 416, 233 (2002).

[6] S. A. Diddams, J. C. Bergquist, S. R. Jefferts, and C. W. Oates, Science 306, 1318 (2004).

[7] P. Gill, G. P. Barwood, H. A. Klein, G. Huang, S. A. Webster, P. J. Blythe, K. Hosaka, S. N. Lea, and H. S. Margolis, Meas. Sci. Technol. 14, 1174 (2003).

[8] P. Gill, Metrologia 42, S125 (2005).

[9] H. S. Margolis, J. Phys. B 41, 154017 (2009).

[10] T. F. Gallagher and W. E. Cooke, Phys. Rev. Lett. 42, 835 (1979).

[11] W. M. Itano, L. L. Lewis, and D. J. Wineland, Phys. Rev. A 25, 1233 (1982).

[12] L. Hollberg and J. L. Hall, Phys. Rev. Lett. 53, 230 (1984).

[13] S. G. Porsev and A. Derevianko, Phys. Rev. A 74, 020502(R) (2006).

[14] A. Dalgarno, Advan. Phys. 11, 281 (1962).

[15] R. R. Teachout and R. T. Pack, Atomic Data 3, 195 (1971).

[16] T. M. Miller and B. Bederson, Adv. At. Mol. Phys. 13, 1 (1977).

[17] T. M. Miller, Adv. At. Mol. Phys. 25, 37 (1988).

[18] van Wijngaarden W A, Adv. At. Mol. Opt. Phys. 36, 141 (1996).

[19] K. D. Bonin and V. V. Kresin, Electric dipole polarizabilities of atoms, molecules and clusters (World Scientific, Singapore, 1997).

[20] N. B. Delone and V. P. Krainov, Physics Uspekhi 42, 669 (1999).

[21] P. Schwerdtfeger, Atomic Static Dipole Polarizabilities, chap. 1, p. 1, in 383. (2006).

[22] H. Gould and T. M. Miller, Adv. At. Mol. Opt. Phys. 51, 343 (2005).

[23] S. R. Lundeen, Adv. At. Mol. Opt. Phys. 52, 161 (2005).

[24] T. M. Miller, Atomic and Molecular Polarizabilities, chap. 10, pp. 10-192, vol. 88 of 382 (2007).

[25] C. Lupinetti and A. J. Thakkar, in [383], p. 505.

[26] T. F. Gallagher, Rydberg Atoms (Cambridge University Press, Cambridge, 2005).

[27] J. R. Fuhr and W. L. Wiese, Atomic Transition Probabilities (CRC Press, Boca Raton, Florida, 1995), vol. 76, chap. 10 , p. 128.

[28] J. Mitroy and M. W. J. Bromley, Phys. Rev. A 70, 052503 (2004).

[29] B. Arora, M. Safronova, and C. W. Clark, Phys. Rev. A 76, 052509 (2007).

[30] B. Arora, M. S. Safronova, and C. W. Clark, Phys. Rev.
A 76, 052516 (2007).

[31] M. S. Safronova, W. R. Johnson, and A. Derevianko, Phys. Rev. A 60, 4476 (1999).

[32] U. Volz and H. Schmoranzer, Phys. Scr. T65, 48 (1996).

[33] J. Sansonetti, W. Martin, and S. Young, Handbook of basic atomic spectroscopic data (2005), (version 1.1.2). [Online] Available: http://physics.nist.gov/Handbook [2007, August 29]. National Institute of Standards and Technology, Gaithersburg, MD.

[34] C. E. Moore, Atomic Energy Levels (ChromiumNiobium NSRDS-NBS 35), vol. 2 (US GPO, Washington DC, 1971).

[35] I. S. Lim and P. Schwerdfeger, Phys. Rev. A 70, 062501 (2004).

[36] C. Lupinetti and A. J. Thakkar, J. Chem. Phys. 122, 044301 (2005).

[37] M. S. Safronova and W. R. Johnson, Adv. At. Mol. Opt. Phys. 55, 191 (2008).

[38] A. Hibbert, M. LeDourneuf, and V. K. Lan, J. Phys. B 10, 1015 (1977).

[39] A. Dalgarno and J. T. Lewis, Proc. R. Soc. London Ser. A 233, 70 (1955).

[40] P. Epstein, Phys. Rev. 28, 695 (1926).

[41] I. Waller, Z. Phys. 38, 635 (1926).

[42] G. Wentzel, Z. Phys. 38, 527 (1926).

[43] V. Yakhontov, Phys. Rev. Lett. 91, 093001 (2003).

[44] R. Szmytkowski and K. Mielewczyk, J. Phys. B: At. Mol. Opt. Phys. 37, 3961 (2004).

[45] R. Szmytkowski, Chem. Phys. Lett. 419, 537 (2006).

[46] U. D. Jentschura and M. Haas, Phys. Rev. A 78, 042504 (2008).

[47] H. A. Bethe and E. E. Salpeter, Quantum mechanics of one- and two-electron atoms (Plenum, New York, 1977).

[48] U. Fano and J. W. Cooper, Rev. Mod. Phys. 40, 441 (1968).

[49] C. W. Clark, J. Opt. Soc. Am. B 7, 488 (1990).

[50] W. R. Johnson, D. Kolb, and K. Huang, At. Data Nucl. Data Tables 28, 333 (1983).

[51] W. R. Johnson, Z. W. Liu, and J. Sapirstein, At. Data and Nuclear Data Tables 64, 279 (1996).

[52] W. R. Johnson, S. A. Blundell, and J. Sapirstein, Phys. Rev. A 37, 307 (1988).

[53] M. G. Kozlov and S. G. Porsev, Eur. Phys. J. D 5, 59 (1999).

[54] M. S. Safronova, B. Arora, and C. W. Clark, Phys. Rev. A 73, 022505 (2006).

[55] H. Katori, T. Ido, and M. Kuwata-Gonokami, J. Phys. Soc. Japan 68, 2479 (1999).

[56] A. Derevianko, W. R. Johnson, M. S. Safronova, and J. F. Babb, Phys. Rev. Lett. 82, 3589 (1999).

[57] S. G. Porsev and A. Derevianko, JETP 102, 195 (2006).

[58] N. Bouloufa, A. Crubellier, and O. Dulieu, Physica Scripta Volume T 134, 014014 (2009).

[59] C. J. Kleinman, Y. Hahn, and L. Spruch, Phys. Rev. 165, 53 (1968).

[60] A. D. Dalgarno, G. W. F. Drake, and G. A. Victor, Phys. Rev. 176, 194 (1968).

[61] A. Dalgarno and A. E. Kingston, Proc. R. Soc. Lon- 
don A 259, 424 (1960).

[62] P. W. Langhoff and M. Karplus, J. Opt. Soc. Am. 59, 863 (1969).

[63] J. W. Schmidt, R. M. Gavioso, E. F. May, and M. R. Moldover, Phys. Rev. Lett. 98, 254504 (2007).

[64] D. Goebel and U. Hohm, Phys. Rev. A 52, 3691 (1995).

[65] D. Goebel and U. Hohm, J. Phys. Chem. 100, 7710 (1996).

[66] W. D. Hall and J. C. Zorn, Phys. Rev. A 10, 1141 (1974).

[67] R. W. Molof, H. L. Schwartz, T. M. Miller, and B. Bederson, Phys. Rev. A 10, 1131 (1974).

[68] T. M. Miller and B. Bederson, Phys. Rev. A 14, 1572 (1976).

[69] H. L. Schwartz, T. M. Miller, and B. Bederson, Phys. Rev. A 10, 1924 (1974).

[70] A. D. Cronin, J. Schmiedmayer, and D. E. Pritchard, Rev. Mod. Phys. 81, 1051 (2009).

[71] A. Miffre, M. Jacquet, M. Buchner, G. Trenec, and J. Vigue, Eur. Phys. J. D 38, 353 (2006).

[72] C. R. Ekstrom, J. Schmiedmayer, M. S. Chapman, T. D. Hammond, and D. E. Pritchard, Phys. Rev. A 51, 3883 (1995).

[73] W. F. Holmgren, M. C. Revelle, V. P. A. Lonij, and A. Cronin (2010), 1001.3888v1.

[74] J. M. Amini and H. Gould, Phys. Rev. Lett. 91, 153001 (2003).

[75] M. A. Kadar-Kallen and K. D. Bonin, Phys. Rev. Lett. 68, 2015 (1992).

[76] M. A. Kadar-Kallen and K. D. Bonin, Phys. Rev. Lett. 72, 828 (1994).

[77] M. Hu and B. R. Kusse, Phys. Rev. A 66, 062506 (2002).

[78] G. S. Sarkisov, I. L. Beigman, V. P. Shevelko, and K. W. Struve, Phys. Rev. E 73, 042501 (2006).

[79] M. Born and W. Heisenberg, Z. Phys. 407, 407 (1924).

[80] J. E. Mayer and M. G. Mayer, Phys. Rev. 43, 605 (1933).

[81] A. Dalgarno and J. T. Lewis, Proc. Phys. Soc. London Ser. A 69, 57 (1956).

[82] R. J. Drachman, Phys. Rev. A 26, 1228 (1982).

[83] R. J. Drachman and A. K. Bhatia, Phys. Rev. A 51, 2926 (1995).

[84] J. Mitroy and M. S. Safronova, Phys. Rev. A 79, 012513 (2009).

[85] K. Bockasten, Phys. Rev. A 9, 1087 (1974).

[86] G. W. F. Drake and R. A. Swainson, Phys. Rev. A 44, 5448 (1991)

[87] R. A. Swainson and G. W. F. Drake, Can. J. Phys. 70, 187 (1992).

[88] J. Mitroy, Phys. Rev. A 78, 052515 (2008).

[89] J. Stark, Annalen. der Physik 43, 965 (1913).

[90] L. R. Hunter, D. Krause, Jr., S. Murthy, and T. W. Sung, Phys. Rev. A 37, 3283 (1988).

[91] L. R. Hunter, D. Krause, D. J. Berkeland, and M. G. Boshier, Phys. Rev. A 44, 6140 (1991).

[92] L. R. Hunter, D. Krause, K. E. Miller, D. J. Berkeland, and M. G. Boshier, Opt. Commun. 94, 210 (1992).

[93] S. C. Bennett, J. L. Roberts, and C. E. Wieman, Phys. Rev. A 59, R16 (1999).

[94] W. A. van Wijngaarden, in American Institute of Physics Conference Series, edited by W. E. Baylis and G. W. F. Drake (1999), vol. 477 of American Institute of Physics Conference Series, pp. 305-321.

[95] J. A. Sherman, T. W. Koerber, A. Markhotok,
W. Nagourney, and E. N. Fortson, Phys. Rev. Lett. 94, 243001 (2005).

[96] E. Iskrenova-Tchoukova and M. S. Safronova, Phys. Rev. A 78, 012508 (2008).

[97] T. Rosenband, W. M. Itano, P. O. Schmidt, D. B. Hume, J. C. J. Koelemeij, J. C. Bergquist, and D. J. Wineland, Proceedings of the 20th European Frequency and Time Forum, PTB Braunschweig, Germany, 2006. p. 289 (2006).

[98] R. Sánchez, M. Žáková, Z. Andjelkovic, B. A. Bushaw, K. Dasgupta, G. Ewald, C. Geppert, H. Kluge, J. Krämer, M. Nothhelfer, et al., New Journal of Physics 11, 073016 (2009).

[99] A. Hibbert, Rep. Prog. Phys. 38, 1217 (1975).

[100] V. A. Dzuba, V. V. Flambaum, and M. G. Kozlov, Phys. Rev. A 54, 3948 (1996).

[101] C. Froese Fischer, T. Brage, and P. Jönsson, Computational Atomic Structure (An MCHF Approach) (Institute of Phyics Publishing, Bristol, 1997).

[102] I. P. Grant, Relativistic Quantum Theory of Atoms and Molecules Theory and Computation (Springer, New York, 2007).

[103] C. Laughlin and G. A. Victor, Adv. At. Mol. Phys. 25, 163 (1988).

[104] W. Müller, J. Flesch, and W. Meyer, J. Chem. Phys. 80, 3297 (1984).

[105] J. Mitroy and M. W. J. Bromley, Phys. Rev. A 68, 052714 (2003).

[106] G. A. Victor, R. F. Stewart, and C. Laughlin, Astrophys. J. Suppl. Ser. 31, 237 (1976).

[107] D. W. Norcross and M. J. Seaton, J. Phys. B 9, 2983 (1976).

[108] R. Santra, K. V. Christ, and C. H. Greene, Phys. Rev. A 69, 042510 (2004).

[109] J. Migdalek and W. E. Baylis, J. Phys. B 11, L497 (1978).

[110] S. Hameed, A. Herzenberg, and M. G. James, J. Phys. B 1, 822 (1968).

[111] S. Hameed, J. Phys. B 5, 746 (1972).

[112] H. L. Zhou and D. W. Norcross, Phys. Rev. A 40, 5048 (1989).

[113] A. Ye and G. Wang, Phys. Rev. A 78, 014502 (2008).

[114] G. D. Doolen, Crc handbook of chemistry and physics (1984), unpublished, referenced in [24].

[115] X. Chu and A. Dalgarno, J. Chem. Phys. 121, 4083 (2004).

[116] K. Pachucki and J. Sapirstein, Phys. Rev. A 63, 012504 (2001).

[117] Z. C. Yan, J. F. Babb, A. Dalgarno, and G. W. F. Drake, Phys. Rev. A 54, 2824 (1996).

[118] L.-Y. Tang, Z.-C. Yan, T.-Y. Shi, and J. F. Babb, Phys. Rev. A 79, 062712 (2009).

[119] G. Łach, B. Jeziorski, and K. Szalewicz, Phys. Rev. Lett. 92, 233001 (2004).

[120] Y. Suzuki and K. Varga, Stochastic variational Approach to Quantum-Mechanical Few-Body Problems, 172 (Springer, New York, 1998).

[121] J. Komasa, Phys. Rev. A 65, 012506 (2002).

[122] G. E. Brown and D. Ravenhall, Proc. R. Soc. London, Ser. A 208, 552 (1951).

[123] C. Cannon and A. Derevianko, Phys. Rev. A 69, 030502(R) (2004).

[124] U. I. Safronova and M. S. Safronova, Phys. Rev. A 79, 022512 (2009). 
[125] V. A. Dzuba, V. V. Flambaum, and O. P. Sushkov, Phys. Lett. A 140, 493 (1989).

[126] V. A. Dzuba, V. V. Flambaum, and J. S. M. Ginges, Phys. Rev. A 63, 062101 (2001).

[127] V. A. Dzuba, V. V. Flambaum, and J. S. M. Ginges, Phys. Rev. D 66, 076013 (2002).

[128] V. A. Dzuba, Phys. Rev. A 78, 042502 (2008).

[129] E. J. Angstmann, V. A. Dzuba, and V. V. Flambaum, Phys. Rev. Lett. 97, 040802 (2006).

[130] E. J. Angstmann, V. A. Dzuba, and V. V. Flambaum, Phys. Rev. A 74, 023405 (2006).

[131] F. Coester and H. Kümmel, Nucl. Phys. 17, 477 (1960).

[132] I. S. Lim, P. Schwerdtfeger, B. Metz, and H. Stoll, J. Chem. Phys. 122, 104103 (2005).

[133] A. J. Sadlej, M. Urban, and O. Gropen, Phys. Rev. A 44, 5547 (1991).

[134] S. Schäfer, M. Mehring, R. Schäfer, and P. Schwerdtfeger, Phys. Rev. A 76, 052515 (2007).

[135] S. A. Blundell, W. R. Johnson, Z. W. Liu, and J. Sapirstein, Phys. Rev. A 39, 3768 (1989).

[136] S. A. Blundell, W. R. Johnson, Z. W. Liu, and J. Sapirstein, Phys. Rev. A 40, 2233 (1989).

[137] R. Pal, M. S. Safronova, W. R. Johnson, A. Derevianko, and S. G. Porsev, Phys. Rev. A 75, 042515 (2007).

[138] S. G. Porsev and A. Derevianko, Phys. Rev. A 73, 012501 (2006).

[139] M. S. Safronova, M. G. Kozlov, W. R. Johnson, and D. Jiang, Phys. Rev. A 80, 012516 (2009).

[140] S. G. Porsev, M. G. Kozlov, Y. G. Rakhlina, and A. Derevianko, Phys. Rev. A 64, 012508 (2001).

[141] S. G. Porsev, A. D. Ludlow, M. M. Boyd, and J. Ye, Phys. Rev. A 78, 032508 (2008).

[142] V. A. Dzuba and J. S. Ginges, Phys. Rev. A 73, 032503 (2006).

[143] V. A. Dzuba and A. Derevianko, J. Phys. B 43, 074011 (2010).

[144] V. Dzuba, M. Kozlov, S. Porsev, and V. Flambaum, Zh. Eksp. Theor. Fiz. 114, 1636 (1998).

[145] H. Hachisu, K. Miyagishi, S. G. Porsev, A. Derevianko, V. D. Ovsiannikov, V. G. Pal'Chikov, M. Takamoto, and H. Katori, Phys. Rev. Lett. 100, 053001 (2008), 0711.4638 .

[146] D. Nikolić and E. Lindroth, J. Phys. B 37, L285 (2004).

[147] P. Soldán, E. P. F. Lee, and T. G. Wright, Physical Chemistry Chemical Physics (Incorporating Faraday Transactions) 3, 4661 (2001).

[148] T. Nakajima and K. Hirao, Chemistry Lett. 30, 706 (2001).

[149] K. Hald, F. Pawłowski, P. Jørgensen, and C. Hättig, J. Chem. Phys. 118, 1292 (2003).

[150] A. J. Thakkar, H. Hettema, and P. E. S. Wormer, J. Chem. Phys. 97, 3252 (1992).

[151] R. Franke, H. Müller, and J. Noga, J. Chem. Phys. 114, 7746 (2001).

[152] D. Gugan and G. W. Michel, Molec. Phys. 39, 783 (1980).

[153] D. Gugan and G. W. Michel, Metrologia 16, 149 (1980).

[154] R. H. Orcutt and R. H. Cole, J. Chem. Phys. 46, 697 (1967).

[155] A. C. Newell and R. C. Baird, J. Appl. Phys. 36, 3751 (1965).

[156] A. K. Bhatia and R. J. Drachman, Can. J. Phys. 75, 11 (1997).

[157] W. R. Johnson and K. T. Cheng, Phys. Rev. A 53, 1375
(1996).

[158] I. S. Lim, J. K. Laerdahl, and P. Schwerdtfeger, J. Chem. Phys. 116, 172 (2002).

[159] W. E. Cooke, T. F. Gallagher, R. M. Hill, and S. A. Edelstein, Phys. Rev. A 16, 1141 (1977).

[160] U. Öpik, Proc. Phys. Soc. London 92, 566 (1967).

[161] I. Johansson, Ark. Fys. 20, 135 (1960).

[162] K. A. Safinya, T. F. Gallagher, and W. Sandner, Phys. Rev. A 22, 2672 (1980).

[163] R. R. Freeman and D. Kleppner, Phys. Rev. A 14, 1614 (1976).

[164] L. J. Curtis and P. S. Ramanujam, J. Opt. Soc. Am. B 71, 1315 (1981).

[165] L. G. Gray, X. Sun, and K. B. MacAdam, Phys. Rev. A 38, 4985 (1988).

[166] K.-H. Weber and C. J. Sansonetti, Phys. Rev. A 35, 4650 (1987).

[167] K. Bockasten, Phys. Rev. 102, 729 (1956).

[168] L. Hamonou and A. Hibbert, J. Phys. B 40, 3555 (2007).

[169] M. S. Safronova and C. W. Clark, Phys. Rev. A 69, 040501(R) (2004).

[170] L.-Y. Tang, Z.-C. Yan, T.-Y. Shi, and J. Mitroy, Phys. Rev. A 10X, "in press" (2010), 1001.4116v1.

[171] J. Y. Zhang, J. Mitroy, and M. W. J. Bromley, Phys. Rev. A. 75, 042509 (2007).

[172] G. Maroulis, J. Chem. Phys. 121, 10519 (2004).

[173] I. S. Lim, M. Pernpointner, M. Seth, J. K. Laerdahl, P. Schwerdtfeger, P. Neogrady, and M. Urban, Phys. Rev. A 60, 2822 (1999).

[174] A. J. Thakkar and C. Lupinetti, Chem. Phys. Lett. 402, 270 (2005).

[175] W. R. Johnson, U. I. Safronova, A. Derevianko, and M. S. Safronova, Phys. Rev. A 77, 022510 (2008).

[176] U. I. Safronova and M. S. Safronova, Phys. Rev. A 78, 052504 (2008).

[177] E. Iskrenova-Tchoukova, M. S. Safronova, and U. I. Safronova, J. Comput. Methods Sci. Eng. 7, 521 (2007).

[178] U. I. Safronova, W. R. Johnson, and M. S. Safronova, Phys. Rev. A 76, 042504 (2007).

[179] J. Y. Zhang and J. Mitroy, Phys. Rev. A 76, 022705 (2007).

[180] C. Zhu, A. Dalgarno, S. G. Porsev, and A. Derevianko, Phys. Rev. A. 70, 032722 (2004).

[181] L. Windholz, M. Musso, G. Zerza, and H. Jager, Phys. Rev. A 46, 5812 (1992).

[182] L. Windholz and M. Musso, Phys. Rev. A 39, 2472 (1989).

[183] C. Krenn, W. Scherf, O. Khait, M. Musso, and L. Windholz, Z. Phys. D 41, 229 (1997).

[184] P. Hannaford, W. R. MacGillivray, and M. C. Standage, J. Phys. B 12, 4033 (1979).

[185] M. Kawamura, W. Jin, N. Takahasi, and T. Minowi, J. Phys. Soc. Japan 78, 034301 (2009).

[186] C. E. Tanner and C. Wieman, Phys. Rev. A 38, 162 (1988).

[187] H.-J. Werner and W. Meyer, Phys. Rev. A 13, 13 (1976).

[188] L.-Y. Tang, J.-Y. Zhang, Z.-C. Yan, T.-Y. Shi, J. F. Babb, and J. Mitroy, Phys. Rev. A 80, 042511 (2009).

[189] Z.-W. Wang and K. T. Chung, J. Phys. B 27, 855 (1994).

[190] J. Mitroy and J. Y. Zhang, Eur. Phys. J D 46, 415 (2008). 
[191] J. Mitroy, J. Y. Zhang, and M. W. J. Bromley, Phys. Rev. A 77, 032512 (2008).

[192] B. Arora, M. S. Safronova, and C. W. Clark, Phys. Rev. A 76, 064501 (2007).

[193] D. Jiang, B. Arora, M. S. Safronova, and C. W. Clark, J. Phys. B 42, 150420 (2009).

[194] B. J. Lyons and T. F. Gallagher, Phys. Rev. A 57, 2426 (1998).

[195] E. S. Chang, J. Phys. B 16, L539 (1983).

[196] T. F. Gallagher, R. Kachru, and N. H. Tran, Phys. Rev. A 26, 2611 (1982).

[197] E. L. Snow, M. A. Gearba, R. A. Komara, S. R. Lundeen, and W. G. Sturrus, Phys. Rev. A 71, 022510 (2005).

[198] E. L. Snow and S. R. Lundeen, Phys. Rev. A 77, 052501 (2008).

[199] E. L. Snow and S. R. Lundeen, Phys. Rev. A 76, 052505 (2007).

[200] C. E. Theodosiou, L. J. Curtis, and C. A. Nicolaides, Phys. Rev. A 52, 3677 (1995).

[201] K. E. Miller, D. Krause Jr., and L. R. Hunter, Phys. Rev. A 49, 5128 (1994).

[202] L. Windholz and C. Neureiter, Phys. Lett. A 109, 155 (1985).

[203] R. Ashby, J. J. Clarke, and W. A. van Wijngaarden, Eur. Phys. J. D 23, 327 (2003).

[204] K. C. Harvey, R. T. Hawkins, G. Meisel, and A. L. Schawlow, Phys. Rev. Lett. 34, 1073 (1975).

[205] R. W. Schmieder, A. Lurio, and W. Happer, Phys. Rev. A 3, 1209 (1971).

[206] A. Khadjavi, A. Lurio, and W. Happer, Phys. Rev. 167, 128 (1968).

[207] W. van Wijngaarden, J. Quant. Spect. Rad. Transf. 57, 275 (1997).

[208] W. Hogervorst and S. Svanberg, Phys. Src. 12, 67 (1975).

[209] S. Svanberg, Phys. Scr. 5, 132 (1972).

[210] M. Yoshimine and R. P. Hurst, Phys. Rev. 135, 612 (1964).

[211] E. A. Reinsch and W. Meyer, Phys. Rev. A 14, 915 (1976).

[212] F. Maeder and W. Kutzelnigg, Chem. Phys. 42, 95 (1979).

[213] G. Maroulis, Chem. Phys. Lett. 334, 207 (2001).

[214] P. Soldán, M. T. Cvitaš, and J. M. Hutson, Phys. Rev. A 67, 054702 (2003).

[215] A. Dalgarno and A. E. Kingston, Proc. Phys. Soc. London 73, 455 (1959).

[216] M. Gunawardena, D. S. Elliott, M. S. Safronova, and U. Safronova, Phys. Rev. A 75, 022507 (2007).

[217] W. A. van Wijngaarden, E. A. Hessels, J. Li, and N. E. Rothery, Phys. Rev. A 49, 2220 (1994).

[218] W. van Wijngaarden and J. Li, J. Quant. Spect. Rad. Transf. 52, 555 (1994).

[219] G. Khvoshtenko and M. Chaika, Optica. Spectrosk. 25, 246 (1968).

[220] K. Fredriksson and S. Svanberg, Z. Physik. 281, 189 (1977).

[221] J. E. Wessel and D. E. Cooper, Phys. Rev. A 35, 1621 (1987).

[222] J. Xia, J. Clarke, J. Li, and W. van Wijngaarden, Phys. Rev. A 56, 5176 (1997).

[223] M. Auzinsh, K. Blushs, R. Ferber, F. Gahbauer, A. Jarmola, and M. Tamanis, Opt. Commun. 264, 333 (2006).
[224] V. Domelunksen, Optica. Spectrosk. 54, 950 (1983).

[225] M. Auzinsh, K. Bluss, R. Ferber, F. Gahbauer, A. Jarmola, M. S. Safronova, U. I. Safronova, and M. Tamanis, Phys. Rev. A 75, 022502 (2007).

[226] D. Tunega, Chem. Phys. Lett. 269, 435 (1997).

[227] E. F. Archibong and A. J. Thakkar, Phys. Rev. A 44, 5478 (1991)

[228] G. L. Bendazzoli and A. Monari, Chem. Phys. 306, 153 (2004).

[229] L. Hamonou and A. Hibbert, J. Phys. B 41, 245004 (2008).

[230] R. Glass, J. Phys. B 20, 4649 (1987).

[231] N. Reshetnikov, L. J. Curtis, M. S. Brown, and R. E. Irving, Phys. Scr. 77, 015301 (2008).

[232] J. Mitroy and J. Y. Zhang, Phys. Rev. A 76, 062703 (2007).

[233] J. Mitroy and J. Y. Zhang, Mol. Phys. 106, 127 (2008).

[234] J. Mitroy and J. Y. Zhang, J. Chem. Phys. 128, 134305 (2008).

[235] J. Mitroy, J. Y. Zhang, M. W. J. Bromley, and K. G. Rollin, Eur. Phys. J. D 53, 15 (2009).

[236] D. Goebel, U. Hohm, and G. Maroulis, Phys. Rev. A 54, 1973 (1996).

[237] C. Thierfelder and P. Schwerdtfeger, Phys. Rev. A 79, 032512 (2009).

[238] K. T. Tang and J. P. Toennies, Mol. Phys. 106, 1645 (2008).

[239] R. A. Komara, M. A. Gearba, C. W. Fehrenbach, and S. R. Lundeen, J. Phys. B 38, S87 (2005).

[240] S. G. Porsev, Y. G. Rakhlina, and M. G. Kozlov, Phys. Rev. A 60, 2781 (1999).

[241] P. Schwerdtfeger and G. A. Bowmaker, J. Chem. Phys. 100, 4487 (1994).

[242] P. Neogrady, V. Kello, M. Urban, and A. J. Sadlej, Int. J. Quant. Chem. 63, 557 (1997)

[243] M. Ilias and P. Neogrady, Chem. Phys. Lett. 309, 441 (1999).

[244] J. Y. Zhang, J. Mitroy, H. R. Sadeghpour, and M. W. J. Bromley, Phys. Rev. A 78, 062710 (2008).

[245] M. Henderson, L. J. Curtis, R. Matulioniene, D. G. Ellis, and C. E. Theodosiou, Phys. Rev. A 56, 1872 (1997).

[246] L. J. Curtis and C. E. Theodosiou, J. Opt. Soc. Am. B 12, 175 (1995).

[247] P. Neogrady, V. Kello, M. Urban, and A. J. Sadlej, Theor. Chim. Acta 93, 101 (1996).

[248] E. L. Snow and S. R. Lundeen, Phys. Rev. A 75, 062512 (2007).

[249] S. R. Lundeen and C. W. Fehrenbach, Phys. Rev. A 75, 032523 (2007).

[250] C. E. Magnusson and P. O. Zetterberg, Phys. Scr. 15, 237 (1977).

[251] M. G. Kozlov, S. G. Porsev, and W. R. Johnson, Phys. Rev. A 64, 052107 (2001).

[252] A. Hibbert, J. Phys. B 13, 3725 (1980).

[253] T. Fleig, Phys. Rev. A 72, 052506 (2005).

[254] P. Milani, I. Moullet, and W. A. de Heer, Phys. Rev. A 42, 5150 (1990).

[255] T. P. Guella, T. M. Miller, B. Bederson, J. A. D. Stockdale, and B. Jaduszliwer, Phys. Rev. A 29, 2977 (1984).

[256] T. P. Guella, Ph.D. thesis, New York University (1985).

[257] C. Thierfelder, B. Assadollahzadeh, P. Schwerdtfeger, S. Schäfer, and R. Schäfer, Phys. Rev. A 78, 052506 (2008).

[258] J. Bardon and M. Auduffren, J. Physique. (Colloq) C9, 
245 (1984).

[259] A. Kreutzträger and G. v. Oppen, Z. Phys. 265, 421 (1973).

[260] G. von Oppen, Z. Phys. 232, 473 (1970).

[261] S. Yanagimachi, M. Kajiro, M. Machiya, and A. Morinaga, Phys. Rev. A 65, 042104 (2002).

[262] K. Zeiske, G. Zinner, F. Riehle, and J. Helmcke, Appl. Phys. B 60, 205 (1995).

[263] G. von Oppen, Z. Phys. 248, 41 (1971).

[264] G. von Oppen, Z. Phys. 227, 207 (1969).

[265] A. Hese, A. Renn, and H. S. Schweda, Opt. Commun. 20, 385 (1977).

[266] K. A. H. van Leeuwen and W. Hogervorst, Z. Phys. 310, 37 (1983).

[267] C.-H. Li, S. M. Rochester, M. G. Kozlov, and D. Budker, Phys. Rev. A 69, 042507 (2004).

[268] R.-H. Rinkleff, Z. Phys. 291, 23 (1979).

[269] E. J. Robinson, J. Opt. Soc. Am. 59, 782 (1969).

[270] S. Legowski, A. Molhem, G. Osiński, and P. Rudecki, Z. Phys. D 35, 101 (1995).

[271] W. J. Sandle, M. C. Standage, and D. M. Warrington, J. Phys. B 8, 1203 (1975).

[272] R. D. Kaul and W. S. Latshaw, J. Opt. Soc. America 62, 615 (1972).

[273] N. J. Martin, P. G. H. Sandars, and G. K. Woodgate, Proc. R. Soc. London A 305, 139 (1968).

[274] F. R. Petersen, H. G. Palmer, and J. H. Shirly, Bull. Am. Phys. Soc. 13, 1674 (1968).

[275] H. Gould, Phys. Rev. A 14, 922 (1976).

[276] R.-H. Rinkleff, Z. Phys. 296, 101 (1980).

[277] P. Kulina and R.-H. Rinkleff, Z. Phys. 304, 371 (1982).

[278] J. Li and W. A. Van Wijngaarden, J. Phys. B 28, 2559 (1995).

[279] T. Schneider, E. Peik, and C. Tamm, Phys. Rev. Lett. 94, 230801 (2005).

[280] A. K. Bhatia and R. J. Drachman, Phys. Rev. A 45, 7752 (1992).

[281] V. I. Korobov, Phys. Rev. A 61, 064503 (2000).

[282] W. Cencek, K. Szalewicz, and B. Jeziorski, Phys. Rev. Lett. 86, 5675 (2001).

[283] J. Pipin and D. M. Bishop, Phys. Rev.A 47, 4571 (1993).

[284] A. G. Vaidyanathan, W. P. Spencer, J. R. Rubbmark, H. Kuiper, C. Fabre, D. Kleppner, and T. W. Ducas, Phys. Rev. A 26, 3346 (1982).

[285] S. G. Porsev and A. Derevianko, Phys. Rev. A 73, 012501 (2006).

[286] Homer, The Odyssey (circa 900BC).

[287] C. S. Wood, S. C. Bennett, D. Cho, B. P. Masterson, J. L. Roberts, C. E. Tanner, and C. E. Wieman, Science 275, 1759 (1997).

[288] M. G. Kozlov, S. G. Porsev, and I. I. Tupitsyn, Phys. Rev. Lett. 86, 3260 (2001).

[289] R. N. Watts, S. L. Gilbert, and C. E. Wieman, Phys. Rev. A 27, 2769 (1983).

[290] V. Rieger, K. Sengstock, U. Sterr, J. H. Müller, and W. Ertmer, Opt. Commun. 99, 172 (1993).

[291] V. Rieger, Ph.D. thesis, Univerisitat Hannover (1996).

[292] A. Morinaga, M. Nakamura, T. Kurosu, and N. Ito, Phys. Rev. A 54, 21(R) (1996).

[293] J. Li and W. A. van Wijngaarden, Phys. Rev. A 53, 604 (1996).

[294] J. Li and W. A. van Wijngaarden, Phys. Rev. A 51, 3560 (1995).
[295] D. M. Harber and M. V. Romalis, Phys. Rev. A 63 013402 (2001).

[296] D. DeMille, D. Budker, and E. D. Commins, Phys. Rev. A 50, 4657 (1994).

[297] M. S. Safronova, W. R. Johnson, U. I. Safronova, and T. E. Cowan, Phys. Rev. A 74, 022504 (2006).

[298] S. C. Doret, P. D. Friedberg, A. J. Speck, D. S. Richardson, and P. K. Majumder, Phys. Rev. A 66, 052504 (2002).

[299] J. Mitroy and D. W. Norcross, Phys. Rev. A 39, 537 (1989).

[300] D. Goebel, U. Hohm, and K. Kerl, J. Mol. Struct. 349, 253 (1995).

[301] M. Yasuda, T. Kishimoto, M. Takamoto, and H. Katori, Phys. Rev. A 73, 011403 (2006).

[302] S. B. Nagel, P. G. Mickelson, A. D. Saenz, Y. N. Martinez, Y. C. Chen, T. C. Killian, P. Pellegrini, and R. Côté, Phys. Rev. Lett. 94, 083004 (2005).

[303] T. Rosenband, C. W. Hume, D B Chou, A. Brusch, L. Lorini, W. H. Oskay, R. E. Drullinger, T. M. Fortier, J. E. Stalnaker, S. A. Diddams, W. C. Swann, et al., Science 319, 1808 (2008).

[304] M. W. J. Bromley and J. Mitroy, Phys. Rev. A 65, 062506 (2002).

[305] S. Topcu, J. Nasser, L. M. L. Daku, and S. Fritzsche, Phys. Rev. A 73, 042503 (2006).

[306] R. A. Komara, M. A. Gearba, S. R. Lundeen, and C. W. Fehrenbach, Phys. Rev. A 67, 062502 (2003).

[307] R.-H. Rinkleff and F. Thorn, Z. Phys. D 31, 31 (1994).

[308] B. Schuh, C. Neureiter, H. Jäger, and L. Windholz, Z. Phys. D 37, 149 (1996).

[309] A. Derevianko, Phys. Rev. A 65, 012106 (2002).

[310] M. S. Safronova, C. J. Williams, and C. W. Clark, Phys. Rev. A 69, 022509 (2004).

[311] D. Jiang, B. Arora, and M. S. Safronova, Phys. Rev. A 78, 022514 (2008).

[312] S. G. Porsev, K. Beloy, and A. Derevianko, Phys. Rev. Lett. 102, 181601 (2009).

[313] V. A. Dzuba, V. V. Flambaum, and O. P. Sushkov, Phys. Rev. A 56, R4357 (1997).

[314] A. A. Vasilyev, I. M. Savukov, M. S. Safronova, and H. Berry, Phys. Rev. A 66, 020101R (2002).

[315] S. A. Blundell, J. Sapirstein, and W. R. Johnson, Phys. Rev. D 45, 1602 (1992).

[316] D. Cho, C. S. Wood, S. C. Bennett, J. L. Roberts, and C. E. Wieman, Phys. Rev. A 55, 1007 (1997).

[317] W. C. Haxton and C. E. Wieman, Ann. Rev. Nucl. Part. Sci. 51, 261 (2001).

[318] W. C. Haxton, C.-P. Liu, and M. J. Ramsey-Musolf, Phys. Rev. Lett. 86, 5247 (2001).

[319] M. S. Safronova, R. Pal, D. Jiang, M. Kozlov, W. Johnson, and U. I. Safronova, Nucl. Phys. A 827, 411c (2009).

[320] J. McKeever, J. R. Buck, A. D. Boozer, A. Kuzmich, H.-C. Nagerl, D. M. Stamper-Kurn, and H. J. Kimble, Phys. Rev. Lett. 90, 133602 (2003).

[321] D. Jaksch, H.-J. Briegel, J. I. Cirac, C. W. Gardiner, and P. Zoller, Phys. Rev. Lett. 82, 1975 (1999).

[322] L. Isenhower, E. Urban, X. L. Zhang, A. T. Gill, T. Henage, T. A. Johnson, T. G. Walker, and M. Saffman, Phys. Rev. Lett. 104, 010503 (2010).

[323] M. Safronova, C. J. Williams, and C. W. Clark, Phys. Rev. A 67, 040303 (2003).

[324] M. Saffman and T. G. Walker, Phys. Rev. A 72, 22347 
(2005).

[325] A. V. Gorshkov, A. M. Rey, A. J. Daley, M. M. Boyd, J. Ye, P. Zoller, and M. D. Lukin, Phys. Rev. Lett. 102, 110503 (2009).

[326] M. Chwalla, J. Benhelm, K. Kim, G. Kirchmair, T. Monz, M. Riebe, P. Schindler, A. S. Villar, W. Hänsel, C. F. Roos, et al., Phys. Rev. Lett. 102, $023002(2009)$.

[327] P. Dubé, A. A. Madej, J. E. Bernard, and A. D. Shiner, in Society of Photo-Optical Instrumentation Engineers (SPIE) Conference Series (2007), vol. 6673 of Society of Photo-Optical Instrumentation Engineers (SPIE) Conference Series.

[328] W. H. Oskay, S. A. Diddams, E. A. Donley, T. M. Fortier, T. P. Heavner, L. Hollberg, W. M. Itano, S. R. Jefferts, M. J. Delaney, K. Kim, et al., Phys. Rev. Lett. 97, 020801 (2006).

[329] K. Hosaka, S. A. Webster, A. Stannard, B. R. Walton, H. S. Margolis, and P. Gill, Phys. Rev. A 79, 033403 (2009).

[330] E. Biémont, J. Dutrieux, I. Martin, and P. Quinet, J. Phys. B 31, 3321 (1998).

[331] S. N. Lea, S. A. Webster, and G. P. Barwood, Proceedings of the 20th European Frequency and Time Form, PTB Braunschweig, Germany 85, 302 (2006).

[332] C. Tamm, B. Lipphardt, H. Schnatz, R. Wyands, S. Weyers, T. Schneider, and E. Peik, IEEE Trans. Instrum. Measur. 56, 601 (2007).

[333] T. Rosenband, P. O. Schmidt, D. B. Hume, W. M. Itano, T. M. Fortier, J. E. Stalnaker, K. Kim, S. A. Diddams, J. C. J. Koelemeij, J. C. Bergquist, et al., Phys. Rev. Lett. 98, 220801 (2007).

[334] T. Becker, J. V. Zanthier, A. Y. Nevsky, C. Schwedes, M. N. Skvortsov, H. Walther, and E. Peik, Phys. Rev. A 63, 051802 (2001).

[335] M. Eichenseer, A. Y. Nevsky, C. Schwedes, J. von Zanthier, and H. Walther, J. Phys. B 36, 553 (2003).

[336] Y. Ralchenko, A. Kramida, J. Reader, and NIST ASD Team, NIST Atomic Spectra Database Version 3.1.5 (2008), URL http://physics.nist.gov/asd3

[337] J. Friebe, A. Pape, M. Riedmann, K. Moldenhauer, T. Mehlstäubler, N. Rehbein, C. Lisdat, E. M. Rasel, W. Ertmer, H. Schnatz, et al., Phys. Rev. A 78, 033830 (2008).

[338] G. K. Campbell, A. D. Ludlow, S. Blatt, J. W. Thomsen, M. J. Martin, M. H. G. de Miranda, T. Zelevinsky, M. M. Boyd, J. Ye, S. A. Diddams, et al., Metrologia 45, 539 (2008).

[339] N. Poli, Z. W. Barber, N. D. Lemke, C. W. Oates, L. S. Ma, J. E. Stalnaker, T. M. Fortier, S. A. Diddams, L. Hollberg, J. C. Bergquist, et al., Phys. Rev. A 77, 050501 (2008).

[340] S. G. Porsev and A. Derevianko, Phys. Rev. A 69, 042506 (2004).

[341] G. Wang and A. Ye, Phys. Rev. A 76, 043409 (2007).

[342] M. C. Bigeon, J. Phys. France 28, 51 (1967).

[343] B. N. Taylor and A. Thompson, The International System of Units (SI) (National Institute of Standards and Technology, Special Publication 330, Gaithersburg, MD, 2008).

[344] A. Wallard, Metrologia 43, 175 (2006).

[345] H. Katori, M. Takamoto, V. G. Pal'chikov, and V. D. Ovsiannikov, Phys. Rev. Lett. 91, 173005 (2003).

[346] M. Takamoto, F.-L. Hong, R. Higashi, and H. Katori,
Nature 435, 321 (2005).

[347] A. D. Ludlow, T. Zelevinsky, G. K. Campbell, S. Blatt, M. M. Boyd, M. H. G. de Miranda, M. J. Martin, J. W. Thomsen, S. M. Foreman, J. Ye, et al., Science 319, 1805 (2008), 0801.4344.

[348] G. Wilpers, C. W. Oates, S. A. Diddams, A. Bartels, T. M. Fortier, W. H. Oskay, J. C. Bergquist, S. R. Jefferts, T. P. Heavner, T. E. Parker, et al., Metrologia 44, 146 (2007).

[349] M. M. Boyd, A. D. Ludlow, S. Blatt, S. M. Foreman, T. Ito, T. Zelevinsky, and J. Ye, Phys. Rev. Lett.. 98, 083002 (2007).

[350] A. V. Taichenachev, V. I. Yudin, V. D. Ovsiannikov, V. G. Pal'Chikov, and C. W. Oates, Phys. Rev. Lett. 101, 193601 (2008).

[351] H. Katori, K. Hashiguchi, E. Y. Il'Inova, and V. D. Ovsiannikov, Phys. Rev. Lett. 103, 153004 (2009).

[352] J. R. Mowat, Phys. Rev. A 5, 1059 (1972).

[353] M. S. Safronova, D. Jiang, B. Arora, C. W. Clark, M. G. Kozlov, U. I. Safronova, and W. R. Johnson, IEEE Trans. Ultrason. Ferroelectrics and Frequency Control 57, 94 (2010)

[354] M. S. Safronova and U. I. Safronova (2010), to be submitted to Phys. Rev. A (2010).

[355] K. Beloy, U. I. Safronova, and A. Derevianko, Phys. Rev.Lett. 97, 040801 (2006).

[356] E. Simon, P. Laurent, and A. Clairon, Phys. Rev. A 57, 436 (1998).

[357] A. Godone, D. Calonico, F. Levi, S. Micalizio, and C. Calosso, Phys. Rev. A 71, 063401 (2005)

[358] M. Marinescu, H. R. Sadeghpour, and A. Dalgarno, Phys. Rev. A 49, 982 (1994).

[359] A. Derevianko, J. F. Babb, and A. Dalgarno, Phys. Rev. A. 63, 052704 (2001).

[360] A. Pashov, P. Popov, H. Knockel, and E. Tiemann, Eur. Phys. J. D 46, 241 (2008).

[361] E. G. M. van Kempen, S. J. J. M. F. Kokkelmans, D. J. Heinzen, and B. J. Verhaar, Phys. Rev. Lett. 88, 093201 (2002).

[362] C. Chin, V. Vuletić, A. J. Kerman, S. Chu, E. Tiesinga, P. J. Leo, and C. J. Williams, Phys. Rev. A 70, 032701 (2004).

[363] R. J. Le Roy and R. B. Bernstein, J. Mol. Spectrosc. 37, 109 (1971).

[364] A. Marte, T. Volz, J. Schuster, S. Durr, G. Rempe, E. G. M. van Kempen, and B. J. Verhaar, Phys. Rev. Lett. 89, 283202 (2002).

[365] Z. C. Yan, J. M. Zhu, and B. L. Zhou, Phys. Rev. A 62, $034501(2000)$.

[366] J. Y. Zhang, Z. C. Yan, D. Vrinceanu, and H. R. Sadeghpour, Phys. Rev. A. 71, 032712 (2005).

[367] J. Y. Zhang, Z. C. Yan, D. Vrinceanu, J. F. Babb, and H. R. Sadeghpour, Phys. Rev. A. 73, 022710 (2006).

[368] J. Y. Zhang, Z. C. Yan, D. Vrinceanu, J. F. Babb, and H. R. Sadeghpour, Phys. Rev. A. 74, 014704 (2006).

[369] J. Y. Zhang, Z. C. Yan, D. Vrinceanu, J. F. Babb, and H. R. Sadeghpour, Phys. Rev. A 76, 012723 (2007).

[370] M. Marinescu and A. Dalgarno, Phys. Rev. A 52, 311 (1995).

[371] M. Marinescu and A. Dalgarno, Z. Phys. D 36, 239 (1996).

[372] M. Marinescu, Phys. Rev. A 56, 4764 (1997).

[373] M. Marinescu and H. R. Sadeghpour, Phys. Rev. A 59, 390 (1999). 
[374] J. Mitroy and J. Y. Zhang, Phys. Rev. A 76, 032706 (2007).

[375] S. G. Porsev and A. Derevianko, Phys. Rev. A 65, 020701(R) (2002).

[376] S. G. Porsev and A. Derevianko, J. Chem. Phys. 119, 844 (2003).

[377] P. J. Mohr and B. N. Taylor, Rev. Mod. Phys. 77, 1 (2005).

[378] B. Fellmuth, C. Gaiser, and J. Fischer, Measurement Science and Technology 17, 145 (2006).

[379] J. A. Stone and A. Stejskal, Metrologia 41, 189 (2004).

[380] A. Derevianko and S. G. Porsev, Phys. Rev. A 65,
053403 (2002).

[381] R. J. Rafac and C. E. Tanner, Phys. Rev. A 58, 1087 (1998).

[382] D. R. Lide and H. P. R. Frederikse, eds., CRC Handbook of Chemistry and Physics, vol. 88 (CRC Press, Boca Raton, Florida, 2007).

[383] G. Maroulis, ed., Atoms, Molecules and Clusters in Electric Fields: Theoretical Approaches to the calculation of electric polarizability (World Scientific, London, 2006). 